\title{
Gravitational bremsstrahlung in ultra-planckian collisions
}

\author{
Dmitry Gal'tsov, ${ }^{a}$ Pavel Spirin ${ }^{a}$ and Theodore N. Tomaras ${ }^{b, c}$ \\ ${ }^{a}$ Department of Theoretical Physics, Moscow State University, \\ 119899, Moscow, Russia \\ ${ }^{b}$ CERN, Theory Division, \\ Geneve, Switzerland \\ ${ }^{c}$ Department of Physics and Crete Center for Theoretical Physics, \\ University of Crete, 71003, Heraklion, Greece \\ E-mail: galtsov@physics.msu.ru, salotop@list.ru, \\ tomaras@physics.uoc.gr
}

ABSTRACT: A classical computation of gravitational bremsstrahlung in ultra-planckian collisions of massive point particles is presented in an arbitrary number $d$ of toroidal or noncompact extra dimensions. Our method generalizes the post-linear formalism of General Relativity to the multidimensional case. The total emitted energy, as well as its angular and frequency distribution are discussed in detail. In terms of the gravitational radius $r_{S}$ of the collision energy, the impact parameter $b$ and the Lorentz factor in the CM frame, the leading order radiation efficiency in the Lab frame is shown to be $\epsilon \sim\left(r_{S} / b\right)^{3(d+1)} \gamma_{\mathrm{cm}}$ for $d=0,1$ and $\epsilon \sim\left(r_{S} / b\right)^{3(d+1)} \gamma_{\mathrm{cm}}^{2 d-3}$ for $d \geqslant 2$, up to a known $d$-dependent coefficient and a $\ln \gamma_{\mathrm{cm}}$ factor for $d=2$, while the characteristic frequency of the radiation is $\omega \sim \gamma / b$. The contribution of the low frequency part $(\omega<1 / b)$ of the radiation to the total radiated energy is shown to be negligible for all values of $d$. The domain of validity of the classical result is discussed. Finally, it is shown that within the region of validity of our approach the efficiency can obtain unnatural values greater than one, which is interpreted to mean that the peripheral ultra-planckian collisions should be strongly radiation damped.

KEywords: Large Extra Dimensions, Classical Theories of Gravity, Black Holes

ArXiv EPRINT: 1210.6976 


\section{Contents}

1 Introduction $\quad 1$

2 General setting $\quad 4$

2.1 The model 4

$\begin{array}{ll}2.2 & \text { The iteration scheme }\end{array}$

2.2.1 The asymptotic behavior of the fields 9

2.3 The energy-momentum of the gravitational radiation - formulae 9

$\begin{array}{ll}2.3 .1 & \text { The uncompactified case } M_{1, D-1} \\ \end{array}$

2.3.2 The compactified case $M_{1,3} \times T^{d} \quad 12$

$\begin{array}{ll}2.4 \text { Graviton polarizations } & 14\end{array}$

$\begin{array}{lll}3 & \text { The gravitational radiation source } & \mathbf{1 7}\end{array}$

$\begin{array}{ll}\text { 3.1 The local part }{ }^{1} T_{M N}+{ }^{1} T_{M N}^{\prime} \text { of the source } & 17\end{array}$

$\begin{array}{ll}3.2 \text { The non-local part } S_{M N} \text { of the source } & 19\end{array}$

3.3 Destructive interference of the beamed high-frequency components 21

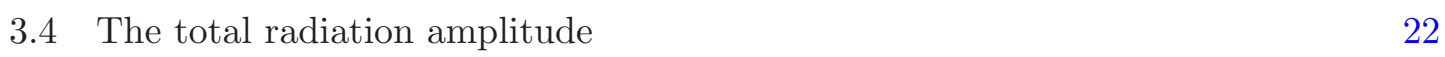

3.4.1 The beamed, high-frequency radiation amplitude 23

3.4.2 Wide-angle, medium-frequency radiation amplitude 24

3.4.3 The beamed, medium-frequency radiation amplitude 25

$\begin{array}{lll}3.5 & \text { Summary } 26\end{array}$

4 The emitted energy $\quad 26$

4.1 The total radiated energy 26

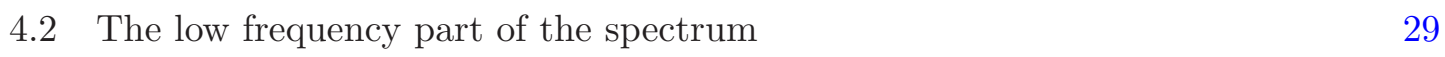

$\begin{array}{ll}4.3 \text { Quantum constraints } & 31\end{array}$

4.3.1 The $\omega \sim \gamma^{2} / b$ and $\omega \sim 1 / b$ regimes $\quad 32$

4.3.2 The $\omega \sim \gamma / b$ regime $\quad 32$

4.4 Radiation emission with characteristic frequency $\omega \sim \gamma / b \quad 33$

4.4.1 $d \geqslant 3 \quad 33$

$\begin{array}{lll}4.4 .2 d=0 & 34\end{array}$

$\begin{array}{lll}4.4 .3 d=1,2 & 34\end{array}$

4.5 Extreme radiation efficiency for $d \geqslant 3$ and $\omega \sim \gamma / b$

5 Conclusions $\quad 35$

$\begin{array}{ll}\text { A Definitions and variations } & \mathbf{3 7}\end{array}$

A.1 Perturbation theory variation over gravitational constant in flat background 37

$\begin{array}{lll}\text { A.2 Useful kinematical formulae } & 38\end{array}$ 
$\begin{array}{ll}\text { B.1 Basic scalar integral } & 39\end{array}$

B.2 Vectorial and tensorial integrals 40

$\begin{array}{lll}\text { B.3 Derived integrals for stresses } & 42\end{array}$

C Integration over frequencies and angles $\quad 44$

D Asymptotic behavior of retarded fields $\quad 45$

\section{Introduction}

The scenario of $\mathrm{TeV}$-scale gravity with large extra dimensions [1-5] is currently being tested experimentally at the LHC [6-11]. In this class of higher-dimensional gravity theories the Planck mass can be of the order of a $\mathrm{TeV}$, provided that the number of extra dimensions is greater than two. If so, the LHC will probe ultra-planckian physics where gravity not only is the dominant force [12], but in addition it is believed to be adequately described by the classical Einstein equations $[13,14]$. This, presumably, allows one to make reliable theoretical predictions of gravitational effects without entering into the complications related to quantum gravity. One such prediction, namely the possibility of black hole formation at the LHC was extensively discussed in the literature during the past ten years [6-11, 15-36].

The main focus of this paper is the study of gravitational bremsstrahlung in ultraplanckian particle collisions. In ultra-planckian collisions with impact parameters smaller than the Schwarzschild radius of the center of mass energy of the colliding particles, the creation of a black hole is a dominant process and is accompanied by radiation losses at the level of 10-40\% depending on the number of extra dimensions (see [37] generalizing earlier calculations by D'Eath and Payne [38-42]). On the other hand, for impact parameters larger than the gravitational radius, the black hole formation process is exponentially suppressed and the gravitational bremsstrahlung becomes the dominant inelastic classical effect, which furthermore can be very strong due to the huge number of light Kaluza-Klein states in the graviton spectrum. It is worth noting, that gravitational radiation may not be maximal in head-on collisions. For instance, radiation from point particles falling into the black hole is minimal for radial infall and grows initially with the impact parameter, a fact which is well known both in four-dimensional general relativity and in models with extra dimensions. The way this is understood is the following: ultra-relativistic particles hitting with some critical impact parameter can be captured into quasi-circular orbits before they plunge into the hole. During these revolutions they radiate with almost constant rate and lose energy more efficiently than during a radial infall $[43,44]$.

A large number of papers from various groups is devoted to the study of gravitational bremsstrahlung in a variety of physical set-ups and using different approaches. A rather incomplete list includes the following: a classical calculation relevant to zero and small 
impact parameter using the colliding wave picture was suggested long ago by D'Eath [3842]. More recently this approach was extended to the extradimensional theories [35-37]. The approach of Smarr et al. in $[45,46]$ was based on the low frequency approximation for the amplitude to estimate the full energy loss. Matzner and Nutku proposed the use of the method of virtual gravitons [47, 48] in analogy with the Weizsäcker-Williams approach in quantum electrodynamics.

Another approach consists in considering radiation in the linearized theory from particles falling towards a black hole [49-53]. Gravitational bremsstrahlung was also studied in the Born approximation of quantum gravity (in four dimensions) [54-56], while the relationship between the Born approximation and the post-linear formalism was discussed in [57]. More recently, a novel approach, making use of perturbative quantum gravity to derive effective equations of motion and to describe gravitational radiation using those, was proposed under the name of "effective theory" approach in [58-66]. Estimates of radiation losses in the eikonal approximation of (multidimensional) quantum gravity were given in $[13,14]$, while Amati, Ciafaloni and Veneziano in a series of papers [67-72] have considered the problem of radiation in ultra-high energy collisions of massless particles in the context of quantum string theory. Estimates of the gravitational bremsstrahlung in high energy collisions in the ADD model were given in [73, 74]. Finally, a lot of numerical work has also been devoted to this subject [75-88].

In four-dimensional theories, in particular, gravitational bremsstrahlung in small-angle ultrarelativistic scattering of point particles was calculated long ago, most notably by Kovacs and Thorne [89-92], in the framework of the so-called "fast motion approximation" scheme, first proposed by Bertotti, Havas and Goldberg [93-100]. A similar independent calculation, using momentum space perturbation theory up to second order in the gravitational constant, was performed in ref. [57] leading to essentially the same results. The latter approach amounts to performing a perturbation expansion of the metric around the Minkowski background up to second order in the gravitational constant and is applicable for arbitrary mass-ratios of the colliding particles. In the case of one mass $M$ much larger than the other $m$, the same result had been obtained earlier by Peters, also using a linear approximation but around the Schwarzschild background of the heavy mass [101-103]. More recent work along these lines can also be found in [104].

The purpose of this paper is to study gravitational radiation losses in the case of ultrarelativistic collisions with large impact parameters, for which the scattering angle is small and calculations can be performed reliably within the post-linear approximation scheme of General Relativity. This paper continues a series of investigations [105-107] devoted to bremsstrahlung in flat space-time arising under non-gravitational scattering of charged particles [105] and scalar radiation in gravity-mediated particle collisions [106]. Here we focus on the problem of gravitational radiation in collisions of massive point particles interacting gravitationally. The computation is purely classical and iterative and, as such, it can only be reliable within a certain domain of validity in the space of parameters. Somewhat unexpectedly, it turns out that, for parameter values within the region of validity of the classical approximation the radiation efficiency, i.e. the fraction of the initial energy which is radiated away, is substantially enhanced compared to the four-dimensional case, 
contrary to earlier qualitative estimates $[108,109]$. Moreover, for a number of extra dimensions greater than two, this quantity grows with a dimension-dependent power of the Lorentz-factor $\gamma$ of the collision.

Specifically, for the leading order radiation efficiency in the laboratory frame we obtain (up to a dimension-dependent numerical coefficient, which however can be quite large, and an inessential $\ln \gamma$ factor for $d=2$ extra dimensions $)^{1}$

$$
\begin{aligned}
& \epsilon \equiv \frac{E}{\mathcal{E}_{0}} \sim\left(\frac{r_{S}}{b}\right)^{3(d+1)} \gamma_{\mathrm{cm}}, \quad d=0,1 \\
& \epsilon \sim\left(\frac{r_{S}}{b}\right)^{3(d+1)} \gamma_{\mathrm{cm}}^{2 d-3}, \quad d \geqslant 2,
\end{aligned}
$$

where $b$ is the impact parameter of the collision, $r_{S}$ is the Schwarzschild radius for the center-of-mass collision energy

$$
r_{S}=\frac{1}{\sqrt{\pi}}\left[\frac{8 \Gamma\left(\frac{d+3}{2}\right)}{d+2}\right]^{\frac{1}{d+1}}\left(\frac{G_{D} \sqrt{s}}{c^{4}}\right)^{\frac{1}{d+1}}
$$

and $\mathcal{E}_{0}=m(\gamma+1) \simeq m \gamma$ is the initial energy in the Lab frame. For collisions of two equal mass particles the Lorentz factors in the Lab and in the center-of-mass frames are related by $\gamma=2 \gamma_{\mathrm{cm}}^{2}-1$.

In the special case of $d=0$ our results for the total emitted energy as well as for its angular and frequency distribution are in perfect agreement with the results of Kovacs and Thorne [89-92].

The present paper is organized in five sections of which this Introduction is the first. In section 2 the model, the basic formulae and the iterative procedure, that will be employed, are described. Also, a complete orthonormal set of graviton polarization tensors in arbitrary dimensions, appropriate for the problem at hand are explicitly constructed. In section 3 the expressions of the local and non-local contributions to the gravitational radiation source are obtained. The phenomenon of destructive interference of the leading local and non-local terms is shown. The total radiation amplitude is also obtained. Section 4 contains the computation of the leading ultra-relativistic order emitted energy in the classical theory in arbitrary dimensions. It also contains a detailed discussion of the frequency and angular distributions of the emitted radiation. The zero frequency limit of the radiation is analyzed in detail in all dimensions. In the same section the domain of validity of the classical computation is discussed. The final result for the radiation efficiency is given and the unnatural possibility for it, to take values greater than one and even diverge in the massless limit for $d \geqslant 2$ extra dimensions, is critically analyzed. A summary together with a few final comments are given in the final Conclusion section.

\footnotetext{
${ }^{1}$ Notice that these expressions differ from the generic formula $\epsilon \sim\left(r_{S} / b\right)^{3(d+1)} \gamma_{\mathrm{cm}}^{2 d+1}$ given erroneously for all dimensions in [107]. The difference is due to an error in [107], related to the extent of the phenomenon of destructive interference discussed in the text. Nevertheless, the qualitative conclusions of [107] are still correct and will be discussed further in section 5 of the present paper.
} 


\section{General setting}

We will study gravitational bremsstrahlung both in uncompactified Minkowski space-time $M_{1, D-1}=M_{1,3} \times \mathbb{R}^{d}$ and in the ADD model $M_{1,3} \times T^{d}$ with the $d$ extra dimensions compactified on a torus. In both cases the collision will be confined on a brane $M_{1,3}$, and the $D$-dimensional cartesian coordinates are split as $x^{M}=\left(x^{\mu} ; y^{i}\right), x^{\mu} \in M_{1,3}, y^{i} \in \mathbb{R}^{d}$ or $T^{d}$, respectively. The brane subspace $M_{1,3}$ is selected by the initial conditions on the particle velocities and is fixed throughout the collision process, since the gravitational interaction cannot expel the particles from the brane.

\subsection{The model}

Consider two point masses $m$ and $m^{\prime}$ moving along the world-lines $x^{M}=z^{M}(\tau)$ and $x^{M}=z^{M}\left(\tau^{\prime}\right)(M=0,1, \ldots, D-1)$ and interacting with the $D$-dimensional gravitational field. The corresponding action is

$$
S=-\sum \frac{1}{2} \int\left(e g_{M N} \dot{z}^{M} \dot{z}^{N}+\frac{m^{2}}{e}\right) d \tau-\frac{1}{\varkappa_{D}^{2}} \int R_{D} \sqrt{-g} d^{D} x
$$

where $\varkappa_{D}^{2} \equiv 16 \pi G_{D}, e(\tau)$ is the einbein of the trajectory and the summation is over the two particles. The metric signature is chosen to be $(+,-, \ldots,-)$ and our convention for the Riemann tensor is $R^{B}{ }_{N R S} \equiv \Gamma_{N S, R}^{B}-\Gamma_{N R, S}^{B}+\Gamma_{N S}^{A} \Gamma_{A R}^{B}-\Gamma_{N R}^{A} \Gamma_{A S}^{B}$, with $\Gamma_{N R}^{A}=$ $(1 / 2) g^{A B}\left(g_{B R, N}+g_{N B, R}-g_{N R, B}\right)$. Finally, the Ricci tensor and curvature scalar are $R_{M N} \equiv \delta_{A}^{B} R_{M B N}^{A}$ and $R \equiv g^{M N} R_{M N}$, respectively.

The action is invariant under the general coordinate reparametrization of spacetime, as well as under the independent reparametrizations of the particle trajectories. For the trajectory of particle $m$ the transformation is $\tau \rightarrow \tilde{\tau}=\tilde{\tau}(\tau)$, under which all fields are scalars except for the einbein $e(\tau)$, which transforms according to $e(\tau) \rightarrow \tilde{e}(\tilde{\tau})=e(\tau) d \tilde{\tau} / d \tau$.

Varying $S$ with respect to $e(\tau)$ and $z^{M}(\tau)$ one obtains the equations

$$
e^{2} g_{M N} \dot{z}^{M} \dot{z}^{N}=m^{2}
$$

and

$$
\frac{d}{d \tau}\left(e \dot{z}^{N} g_{M N}\right)=\frac{e}{2} g_{N P, M} \dot{z}^{N} \dot{z}^{P}
$$

respectively. Two analogous equations are obtained by varying with respect to $e^{\prime}$ and $z^{\prime M}$, while variation of $g_{M N}$ leads to the Einstein equations

$$
G^{M N}=\frac{1}{2} \varkappa_{D}^{2} T^{M N}, \quad T^{M N}=\sum e \int \frac{\dot{z}^{M} \dot{z}^{N} \delta^{D}(x-z(\tau))}{\sqrt{-g}} d \tau
$$

Strictly speaking the notion of point-like particles is not compatible with full non-linear gravity. Nevertheless, it still makes sense in the context of the weak-field perturbation expansion around flat spacetime, which will be adopted here. In this approach one writes the metric as

$$
g_{M N}=\eta_{M N}+\varkappa_{D} h_{M N}
$$


and expands all quantities in powers of $h_{M N}$, using $\eta_{M N}$ to raise and lower the indices. The flat background will be either $D$-dimensional Minkowski space $M_{1, D-1}$, or the product $M_{1,3} \times T^{d}(d=D-4)$ of four-dimensional Minkowski and a $d$-torus.

It is convenient to define the quantity

$$
\psi_{M N} \equiv h_{M N}-\frac{1}{2} h \eta_{M N}, \quad h \equiv \eta^{M N} h_{M N},
$$

and fix the general coordinate reparametrization symmetry by choosing to work in the flat-space harmonic gauge

$$
\partial_{N} \psi^{M N}=0 .
$$

The reparametrization freedom of the particle trajectories will be dealt with later. The expansion of several relevant quantities in powers of $h_{M N}$ is given in appendix A. In particular, the Einstein tensor in the harmonic gauge becomes

$$
G_{M N}=-\frac{\varkappa_{D}}{2} \square \psi_{M N}-\frac{\varkappa_{D}^{2}}{2} S_{M N}+N_{M N},
$$

where $\square=\partial_{M} \partial^{M}$ is the flat d'Alembert operator, $S_{M N}$ is the $\mathcal{O}\left(h^{2}\right)$ part of $G_{M N}$ given by

$$
\begin{gathered}
S_{M N}(h)=h_{M}^{P, Q}\left(h_{N Q, P}-h_{N P, Q}\right)+h^{P Q}\left(h_{M P, N Q}+h_{N P, M Q}-h_{P Q, M N}-h_{M N, P Q}\right)- \\
-\frac{1}{2} h_{, M}^{P Q} h_{P Q, N}-\frac{1}{2} h_{M N} \square h+\frac{1}{2} \eta_{M N}\left(2 h^{P Q} \square h_{P Q}-h_{P Q, L} h^{P L, Q}+\frac{3}{2} h_{P Q, L} h^{P Q, L}\right),
\end{gathered}
$$

while $N_{M N}$ stands for all cubic and higher in $h_{M N}$ terms in the Einstein tensor.

A few remarks are in order. Usually in the ADD scenario one assumes that matter is localized on the brane, i.e. restricted to the subspace $M_{1,3}$. This assumption is noncontradictory within the linearized gravity, since the matter energy momentum in this case is a purely flat-space tensor, whose conservation does not involve gravity. In the full non-linear gravity the $D$-dimensional Bianchi identity

$$
T_{M ; N}^{N}=\frac{1}{\sqrt{-g}}\left[T_{M}^{N} \sqrt{-g}\right]_{, N}-\frac{1}{2} g_{N P, M} T^{N P}=0
$$

implies $D$-dimensional geodesic equations for the particles (2.3) (and similarly for brane fields), which are generally inconsistent with the assumption of matter localization on the brane. For this reason we have to consider the world-lines in (2.1) from the beginning as $D$-dimensional curves. In general, without any extra forces ensuring confinement, the particle motion can be localized on the brane only by suitable initial conditions. However, it will be shown that the gravitational interaction treated perturbatively will not expel the colliding particles from the brane at least to leading order in our iterative scheme. This will be sufficient for the consistency of our computation of bremsstrahlung.

Another technical difference from the standard ADD approach, also related to the fact that our treatment appeals to the $D$-dimensional picture rather than to the fourdimensional one, will be the treatment of the graviton as a $D$-dimensional massless particle, 
instead of as a set of four-dimensional massive fields of spins 2, 1, 0 by suitable rearrangement of the components of the $D$-dimensional graviton $[4,5]$. In particular, it will be more convenient here to choose directly the $D(D-3) / 2$ polarization tensors of the graviton propagating in the bulk, rather than to split them into polarizations of massive particles in four dimensions.

\subsection{The iteration scheme}

Our approach is identical to the one used in simpler models in [105] and [106]. The iterative solution of the above field equations and gauge fixing conditions amounts to formally expanding and computing all fields step-by-step in the form

$$
\Phi={ }^{0} \Phi+{ }^{1} \Phi+{ }^{2} \Phi+\ldots,
$$

where $\Phi$ denotes any of the fields $z^{M}(\tau), z^{\prime M}\left(\tau^{\prime}\right), e(\tau), e^{\prime}\left(\tau^{\prime}\right)$ and $h_{M N}(x)$ and with the left superscript labeling the step of the iteration.

The zeroth order solution is trivial. It describes the two particles moving with constant velocities $\left(u^{M}=\left(u^{\mu}, 0, \ldots, 0\right)\right.$ and $u^{\prime M}=\left(u^{\prime \mu}, 0, \ldots, 0\right)$, respectively $)$ in the $M_{1,3}$ subspace, whose indices are labeled by lower case greek letters $\mu, \nu=0,1,2,3$. Correspondingly, their trajectories ${ }^{0} z^{M}=\left({ }^{0} z^{\mu}(\tau), 0 \ldots, 0\right)$, and similarly for ${ }^{0} z^{\prime M}$, are the straight lines

$$
{ }^{0} z^{\mu}(\tau)=z^{\mu}(0)+u^{\mu} \tau, \quad{ }^{0} z^{\prime \mu}\left(\tau^{\prime}\right)=z^{\prime \mu}(0)+u^{\prime \mu} \tau^{\prime},
$$

in the absence of any gravitational field

$$
{ }^{0} h_{M N}=0 .
$$

The Lagrange multipliers are chosen equal to the corresponding particle masses

$$
{ }^{0} e=m, \quad{ }^{0} e^{\prime}=m^{\prime},
$$

so that the trajectories are parametrized by the corresponding proper times and the fourvelocities satisfy the normalization conditions $\eta_{M N} u^{M} u^{N} \equiv u^{2}=u^{\prime 2}=1$.

We choose to work in the Lorentz frame in which the target particle $m^{\prime}$ is initially at rest and with the projectile $m$ moving along the $z$-axis. Also, we introduce the space-like vector $b^{M}=\left(b^{\mu}, 0, \ldots, 0\right)$ lying on the brane, with:

$$
b^{\mu}=z^{\mu}(0)-z^{\prime \mu}(0)
$$

With no loss of generality one can further assume $(b u)=\left(b u^{\prime}\right)=0$ and choose the $x$-axis along b. Thus,

$$
u^{\mu}=\gamma(1,0,0, v), \quad u^{\prime \mu}=(1,0,0,0), \quad b^{\mu}=(0, b, 0,0),
$$

where $\gamma=1 / \sqrt{1-v^{2}}$ and $b$ is the impact parameter.

The first order correction is obtained next. The zeroth order straight particle trajectories are sources of the first order gravitational field ${ }^{1} h_{M N}\left({ }^{1} h_{M N}^{\prime}\right)$ of each particle, which 
in turn, causes the first order deviation of the trajectory ${ }^{1} z^{\prime M}\left({ }^{1} z^{M}\right)$ of the other one. In the process, the first correction ${ }^{1} e\left({ }^{1} e^{\prime}\right)$ of the einbein fields is also obtained. Explicitly, from the zeroth order trajectories one obtains the zeroth order energy-momentum tensor

$$
{ }^{0} T^{M N}=\sum m \int u u^{M} u^{N} \delta^{D}\left(x-{ }^{0} z(\tau)\right) d \tau,
$$

which in this order has only $\{M, N\}=\{\mu, \nu\}$ components, and from the first order Einstein equations, given in the harmonic gauge by

$$
\square{ }^{1} \psi^{M N}=-\varkappa_{D}{ }^{0} T^{M N},
$$

the first order correction ${ }^{1} \psi^{M N}$ to the metric is obtained. Although only the ${ }^{1} \psi^{\mu \nu}$ components of ${ }^{1} \psi^{M N}$ are non-vanishing, the corresponding ${ }^{1} h^{M N}$ will also have non-vanishing bulk components from the trace part in eq. (2.18).

Using ${ }^{1} h^{M N}$ and the zeroth order solution in equations (2.2) and (2.3) one obtains for ${ }^{1} e$ and ${ }^{1} z^{M}$ the equations ${ }^{2}$

$$
{ }^{1} e=-\frac{m}{2}\left(\varkappa_{D}{ }^{1} h_{M N} u^{M} u^{N}+2 \eta_{M N} u^{M}{ }^{1} \dot{z}^{N}\right)
$$

and

$$
\frac{d}{d \tau}\left({ }^{1} e u_{M}+m^{1} \dot{z}_{M}\right)=-\varkappa_{D} m H_{P Q M} u^{P} u^{Q}, \quad H_{P Q M}={ }^{1} h_{P M, Q}-\frac{1}{2}{ }^{1} h_{P Q, M},
$$

which upon elimination of ${ }^{1} e$ give for ${ }^{1} z^{M}$

$$
\Pi^{M N} \frac{d}{d \tau}{ }^{1} \dot{z}_{N}=-\varkappa_{D} \Pi^{M N} H_{P Q N} u^{P} u^{Q}
$$

where

$$
\Pi^{M N}=\eta^{M N}-u^{M} u^{N}
$$

is the projector onto the subspace orthogonal to $u^{M}$. Equations (2.18), (2.19) and (2.21), together with two similar equations for ${ }^{1} e^{\prime}$ and ${ }^{1} z^{\prime M}$, form a complete set of equations in this order. The gauge fixing condition (2.7) for ${ }^{1} \psi^{M N}$ is a consequence of the conservation of ${ }^{0} T^{M N}$.

It should be kept in mind that in the equation of motion (2.21) of particle $m$ the gravitational field on the right hand side is the one due to $m^{\prime}$. Singularities due to the action on $m$ of its own gravitational field can be removed in lowest order by classical renormalization of the affine parameter of its trajectory [116-118]; this will be enough for our purposes. In what follows we will omit self-action terms and consider only mutual gravitational interaction.

In the next order of iteration one obtains the leading contribution to gravitational radiation. It is due to the accelerated particles, as well as to the cubic gravitational selfinteraction. Indeed, the Einstein field equation reduces for the second order correction ${ }^{2} \psi_{M N}$ of the gravitational field to

$$
{ }^{2} \psi_{M N}=-\varkappa_{D} \tau_{M N},
$$

\footnotetext{
${ }^{2}$ Our gauge condition is $g_{M N} \dot{z}^{M} \dot{z}^{N}=1$. To this order it reduces to ${ }^{1} e=0$.
} 

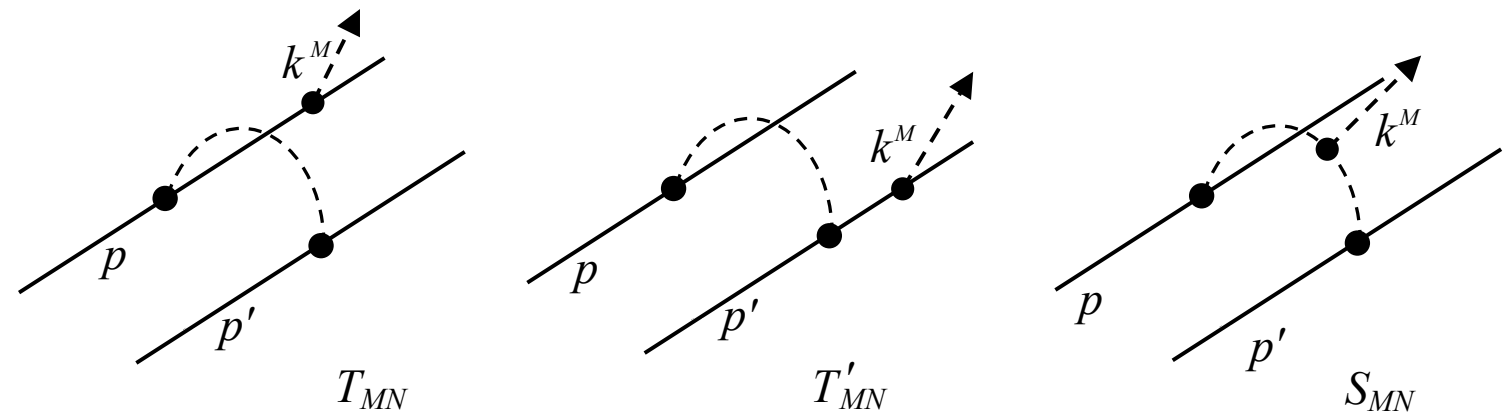

Figure 1. Schematic representation of the sources of gravitational radiation described by (2.24). The interactions are meant to all orders in the quantum perturbation expansion sense. Solid (dotted) lines are colliding particles (gravitons).

with the source term having three contributions:

$$
\tau_{M N}={ }^{1} T_{M N}+{ }^{1} T_{M N}^{\prime}+S_{M N}\left({ }^{1} h\right) .
$$

The first two terms of $\tau_{M N}(2.24)$ represent the particles' contribution to radiation. As obtained to this order from (2.4) they are $^{3}$

$$
\begin{gathered}
{ }^{1} T_{M N}(x)=m \int\left[2{ }^{1} \dot{z}_{(M} u_{N)}+\varkappa_{D}\left(2 u^{P}{ }^{1} h_{P(M} u_{N)}-\frac{{ }^{1} h}{2} u_{M} u_{N}\right)-\right. \\
\left.-u_{M} u_{N}{ }^{1} z^{P} \partial_{P}\right] \delta^{D}\left(x-{ }^{0} z(\tau)\right) d \tau .
\end{gathered}
$$

Ditto for $m^{\prime}$ with $u$ replaced by $u^{\prime}$. To repeat, in $(2.25){ }^{1} h$ is due to $m^{\prime}$. Finally, the part $S_{M N}$ of $\tau_{M N}$ represents the contribution of the gravitational field itself to gravitational radiation. Given that $S_{M N}$ is quadratic in $h_{M N}$, consistency of the iteration to this order requires to substitute $h_{M N} \rightarrow{ }^{1} h_{M N}$ inside $S$. Furthermore, just as the self-interaction terms were ignored in the particles' equations of motion, one has to keep in $S_{M N}\left({ }^{1} h\right)$ only the products of the first order corrections ${ }^{1} h$ due to different particles. The various contributions to the gravitational radiation to this order is shown schematically in figure 1.

It is straightforward to verify on the basis of the equations of motion satisfied by the first order fields that

$$
\partial_{N} \tau^{M N}=0
$$

which guarantees the validity of the gauge fixing condition (2.7) to this order.

So $S_{M N}$ considered as a quadratic form in ${ }^{1} h_{M N}$ constitutes the non-local (in terms of the flat space picture) source of gravitational radiation. This non-locality is due to the non-linearity of the theory and, as will be shown, leads to important differences in the radiation spectrum from linear theories like electromagnetism. More detailed discussions of this point in four dimensions can be found in [57], as well as in its generalization to arbitrary dimension but in a simpler non-linear model in [106].

\footnotetext{
${ }^{3}$ Symmetrization over two indices is defined according to $A_{(M N)} \equiv\left(A_{M N}+A_{N M}\right) / 2$.
} 


\subsubsection{The asymptotic behavior of the fields}

The same tensor $S_{M N}$ considered as a quadratic form in ${ }^{2} h_{M N}$ plays the role of an effective energy-momentum tensor of gravitational waves [112]. This interpretation is based on the conservation equation (2.26) in combination with the asymptotic properties of the solutions of the flat-space d'Alembert equation and is valid in all space-time dimensions.

Recall, that in four dimensions ${ }^{1} h_{\mu \nu}$, which according to (2.18) is due to a static or a uniformly moving mass, falls-off as $1 / r$, and the corresponding "field strength" (derivatives ${ }^{1} h_{\mu \nu, \lambda}$ ) behave as $1 / r^{2}$. On the other hand, the fall-off of the radiative component of the retarded potential ${ }^{2} h_{\mu \nu}$ is $1 / r$ and the same for its derivatives ${ }^{2} h_{\mu \nu, \lambda}$. Correspondingly, in $D$-dimensional Minkowski space-time the behaviors of ${ }^{1} h_{M N}$ and its derivatives ${ }^{1} h_{M N, L}$ are $1 / r^{D-3}$ and $1 / r^{D-2}$, respectively, where $r$ is the radial coordinate in the $D$-1-dimensional space. The corresponding fall-off for the radiation field can be found from the recurrence relations for Green's functions in neighboring dimensions. The derivation looks different in even and odd dimensions, but it leads to the same result, namely (see appendix D)

$$
{ }^{2} h_{M N} \sim{ }^{2} h_{M N, P} \sim \frac{1}{r^{(D-2) / 2}} .
$$

In the case of the ADD model the behavior of the field of a uniformly moving mass depends on the ratio of $r$ to the compactification radius $R$. For $r \ll R$ it has the $D$-dimensional behavior $1 / r^{D-3}$, while for $r \gg R$ it decreases as $1 / r$ (with $r$ being the radial coordinate in three-space). We assume that the observational wave-zone corresponds to this second condition. Then, the fall-off of the radiation field in the wave-zone will be the same as in four dimensions.

Since the effective stress-tensor $S_{M N}\left({ }^{2} h\right)$ is a homogeneous second order function of ${ }^{2} h$ and its derivatives, its fall-off in $D$-dimensional Minkowski will be $1 / r^{(D-2)}$ and in the ADD case $1 / r^{2}$. This is precisely what is needed to get non-zero flux of radiation in the wave zone through the $D$-2-dimensional sphere at spatial infinity of $M_{1, D-1}$, or through the corresponding two-dimensional sphere in the ADD scenario. Note that the use of pseudotensors to define the momentum density of gravitational radiation leads to the same result. Also, note that as a consequence of the above behavior the higher order non-linear terms contained in $N_{M N}$ of eq. (2.8) do not contribute to the radiation energy-momentum.

\subsection{The energy-momentum of the gravitational radiation - formulae}

Following Weinberg [112], we introduce the gravitational energy-momentum $t_{M N} \equiv-2\left(G_{M N}-G_{M N}^{(1)}\right) / \varkappa_{D}^{2}$. Combined with the matter part $T_{M N}$, the total energymomentum of the system is conserved, i.e. it satisfies

$$
\partial^{N}\left(T_{M N}+t_{M N}\right)=0
$$

which is a consequence of the identity $\partial_{N} G^{(1) M N}=0$, and which to second order in the above iteration scheme coincides with (2.26). 


\subsubsection{The uncompactified case $M_{1, D-1}$}

Consider the flat-space world-tube $W$ bounded by two space-like hypersurfaces $\Sigma_{ \pm \infty}$ defined at $t \rightarrow \pm \infty$, and the infinitely far away time-like Cylindrical surface $C$. Thus, the boundary $\partial W$ of $W$ is the union of $\Sigma_{-\infty}, \Sigma_{+\infty}$ and $C$.

Using the energy-momentum conservation equation (2.28) one can write successively for the emitted radiation momentum $\Delta P_{M}$

$$
\begin{aligned}
\Delta P_{M} & =-\int_{\Sigma_{+\infty}} T_{M N} d \sigma^{N}+\int_{\Sigma_{-\infty}} T_{M N} d \sigma^{N}=-\int_{\partial W} T_{M N} d \sigma^{N}=-\int_{W} \partial^{N} T_{M N} d^{D} x \\
& =\int_{W} \partial^{N} t_{M N} d^{D} x=\int_{\partial W} t_{M N} d \sigma^{N}=\int_{C} t_{M N} d \sigma^{N} .
\end{aligned}
$$

To justify the above steps, notice that the first equality is (with $d \sigma^{0}>0$ in both $\Sigma_{-\infty}$ and $\Sigma_{+\infty}$ surfaces) the statement that $\Delta P_{M}$ is minus the change of momentum of the matter system; for the second one uses the fact that the matter system is localized inside the volume and the contribution of $C$ on the surface integral vanishes. Gauss' theorem was used next, together with (2.28) to end up with the surface integral of $t_{M N}$. Finally, for the last equality one used the fact that the surface integrals of $t_{M N}$ on $\Sigma_{-\infty}$ and $\Sigma_{+\infty}$ are zero.

In particular, to the order of our computation in the previous subsection, using Gauss' theorem, equations (2.8), (2.9) and the gauge fixing condition, one may write instead of $(2.29)$

$$
\begin{aligned}
\Delta P_{M} & =\int_{C} S_{M N}\left({ }^{2} h\right) d \sigma^{N}=\int_{W} \partial^{N} S_{M N}\left({ }^{2} h\right) d^{D} x= \\
& =\frac{1}{2} \int_{W}{ }^{2} h_{P Q, M} \square^{2} \psi^{P Q} d^{D} x+\int_{W}{ }^{2} h^{P Q} \square^{2} \psi_{M P, Q} d^{D} x .
\end{aligned}
$$

Furthermore, it is straightforward to show that the last integral on the right vanishes. Indeed,

$$
\begin{aligned}
\int_{W}{ }^{2} h^{P Q} \square^{2} \psi_{M P, Q} d^{D} x & =\int_{C} 2 h^{P Q} \square^{2} \psi_{M P} d \sigma_{Q}-\int_{W}{ }^{2} h^{P Q}{ }_{Q} \square^{2} \psi_{M P} d^{D} x \\
& =-\frac{1}{2} \int_{W}{ }^{2} h^{, P} \square^{2} \psi_{M P} d^{D} x \\
& =-\frac{1}{2} \int_{C}{ }^{2} h \square^{2} \psi_{M P} d \sigma^{P}+\frac{1}{2} \int_{W}{ }^{2} h \square^{2} \psi_{M P}, P
\end{aligned}
$$

where the first integrals of the first and third lines vanish as a consequence of the asymptotic behavior of the fields given in (D.4), (D.5), and (2.27).

Thus, the emitted momentum is

$$
\Delta P_{M}=\frac{1}{2} \int_{W}{ }^{2} h_{P Q, M} \square^{2} \psi^{P Q} d^{D} x .
$$


Using the Fourier transformed quantities

$$
{ }^{2} h^{M N}(x)=\frac{1}{(2 \pi)^{D}} \int{ }^{2} h^{M N}(k) e^{-i k_{Q} x^{Q}} d^{D} k, \quad \tau^{M N}(x)=\frac{1}{(2 \pi)^{D}} \int \tau^{M N}(k) e^{-i k_{Q} x^{Q}} d^{D} k,
$$

one rewrites (2.32) in the form

$$
\Delta P_{M}=-\frac{i \varkappa_{D}^{2}}{2(2 \pi)^{D}} \int k_{M} G_{\mathrm{ret}}(k) \tau_{S N}(k) \tau_{L R}^{*}(k) \tilde{\Lambda}^{S N L R} d^{D} k
$$

where $\tau_{M N}^{*}(k)=\tau_{M N}(-k)$ by the reality of $\tau_{M N}(x)$, and the tensor $\tilde{\Lambda}^{S N L R}$ is given by

$$
\tilde{\Lambda}^{S N L R}=\frac{1}{2}\left[\eta^{S L} \eta^{N R}+\eta^{S R} \eta^{N L}\right]-\frac{1}{D-2} \eta^{S N} \eta^{L R} .
$$

The retarded Green's function is $G_{\text {ret }}=-\mathcal{P}\left(1 / k^{2}\right)+i \pi \epsilon\left(k^{0}\right) \delta\left(k^{2}\right)$. Its real part leads to an integrand, which is odd under parity $k^{M} \rightarrow-k^{M}$ and does not contribute to the integral. Thus, one writes equivalently

$$
\Delta P_{M}=\frac{\varkappa_{D}^{2}}{2(2 \pi)^{D-1}} \int \theta\left(k^{0}\right) k_{M} \tau_{S N}(k) \tau_{L R}^{*}(k) \tilde{\Lambda}^{S N L R} \delta\left(k^{2}\right) d^{D} k .
$$

Taking into account the transversality of $\tau_{M N}\left(k^{N} \tau_{M N}(k)=0\right)$ and the on-shell condition $k^{2}=0$ of the emitted wave, one can replace the Minkowski metric in $\tilde{\Lambda}^{S N L R}$ by

$$
\Delta^{M N} \equiv{ }_{g} \Pi_{L}^{M}{ }_{k^{\prime}} \Pi^{L N}=\eta^{M N}+\frac{k^{M} k^{N}-2(k g) k^{(M} g^{N)}}{(k g)^{2}},
$$

with any time-like unit vector $g$, where $g \Pi=1-g \otimes g$ and $k^{\prime} \Pi=1+k^{\prime} \otimes k^{\prime} /(k g)^{2}$ are projectors onto subspaces transverse to $g$ and $k^{\prime} \equiv{ }_{g} \Pi k=k-(k g) g$, respectively. Since $\left(k^{\prime} g\right)=0$, the projectors ${ }_{g} \Pi$ and $k^{\prime} \Pi$ commute. Their product $\Delta^{M N}$ is then a symmetric projector onto the subspace $M_{k, g}$, perpendicular to $k$ and $g$. By construction, the projector $\Delta$ is idempotent $\left(\Delta^{2}=\Delta\right)$, thus on $M_{k, g}$ it acts as the unit operator. In what follows, we will conveniently choose $g_{M}=u_{M}^{\prime}$ and calculate the flux in the Lorentz frame (the Lab) with $u_{M}^{\prime}=(1,0, \ldots, 0)$.

Thus, $\tilde{\Lambda}$ can equivalently be replaced in (2.36) by

$$
\Lambda^{S N L R}=\frac{1}{2}\left[\Delta^{S L} \Delta^{N R}+\Delta^{S R} \Delta^{N L}\right]-\frac{1}{D-2} \Delta^{S N} \Delta^{L R},
$$

and then upon integration over $|\mathbf{k}|$, one ends up with

$$
\Delta P_{M}=\frac{\varkappa_{D}^{2}}{4(2 \pi)^{D-1}} \int_{0}^{\infty} \omega^{D-3} d \omega \int_{S^{D-2}} d \Omega k_{M} \tau_{S N}(k) \tau_{L R}^{*}(k) \Lambda^{S N L R},
$$

where $\omega \equiv k^{0}=|\mathbf{k}|$. It is easy to show that $\Delta \equiv \eta_{M N} \Delta^{M N}=D-2$. Furthermore, $\Lambda$ satisfies the following relations: 
(a) It is traceless on both pairs of indices

$$
\eta_{S N} \Lambda^{S N L R}=0, \quad \eta_{L R} \Lambda^{S N L R}=0
$$

and (b) it is a projection operator, since it is idempotent $\left(\Lambda^{2}=\Lambda\right)$

$$
\eta_{P S} \eta_{Q T} \Lambda^{M N S T} \Lambda^{P Q L R}=\Lambda^{M N L R}
$$

and projects any second rank symmetric tensor, element of a space we denote by $K$, onto a subspace $K_{\Lambda}$. Acting on a $\tau_{M N}\left(\right.$ or $\left.\tau_{L R}\right)$, ${ }^{4}$ it returns a new symmetric tensor $\tau^{\prime M N}=\Lambda^{M N P Q} \tau_{P Q}$ with two indices. $\Lambda$ acts as a linear operator on the space $K$ of symmetric tensors of rank two. ${ }^{5}$ The properties of $\tau^{\prime}$ determine the space $K_{\Lambda}$.

In particular, the dimensionality of $K_{\Lambda}$ is determined as follows: $\tau_{M N}^{\prime}$ is a symmetric $D \times D$ matrix and thus it has $D(D+1) / 2$ elements. In addition it satisfies the independent conditions $\tau_{M N}^{\prime} u^{\prime N}=0, \tau_{M N}^{\prime} k^{N}=0$ and $\eta^{M N} \tau_{M N}^{\prime}=0$, which impose ${ }^{6} D+(D-1)+1=$ $2 D$ conditions, leaving a total of $D(D-3) / 2$ independent elements in $\tau^{\prime}$. Thus, the dimensionality of $K_{\Lambda}$ is $D(D-3) / 2$, the same as the number of independent polarizations of the graviton in $D$-dimensions.

Given that $\Lambda$ acts on $K_{\Lambda}$ as the unit operator one can write it (decomposition of unity) in terms of an orthonormal basis tensors $\left\{\varepsilon_{\mathcal{P}}^{M N}\right\}$, with $\mathcal{P}=1,2, \ldots, D(D-3) / 2$ of $K_{\Lambda}$

$$
\Lambda^{M N L R}=\sum_{\mathcal{P}} \varepsilon_{\mathcal{P}}^{M N} \varepsilon_{\mathcal{P}}^{L R} .
$$

Using this expression for $\Lambda$, equation (2.39) takes the form

$$
\Delta P_{M}=\frac{\varkappa_{D}^{2}}{4(2 \pi)^{D-1}} \sum_{\mathcal{P}} \int_{0}^{\infty} \omega^{D-3} d \omega \int_{S^{D-2}} d \Omega k_{M}\left|\tau_{L R}(k) \varepsilon_{\mathcal{P}}^{L R}\right|^{2},
$$

i.e. a sum of independent contributions one for each polarization.

\subsubsection{The compactified case $M_{1,3} \times T^{d}$}

Following the same steps as in $M_{1, D-1}$, one is led to a similar expression for the emitted momentum in the ADD scenario. Consider the flat-space world-tube $W$ bounded by two space-like hypersurfaces $\Sigma_{ \pm \infty}$ defined at $t \rightarrow \pm \infty$, and the infinitely distant time-like surface $S_{r \rightarrow \infty}$, defined for $-\infty<t<\infty$, and whose intersection with the space-like hypersurfaces $t=$ const will be denoted by $B_{t}$. According to our convention all quantities constructed from the metric perturbation $h_{M N}$ are flat-space tensors, so the covariant derivatives acting on them are simply partial derivatives. Starting with the energy-momentum conservation (2.28), and following the same steps as above, one concludes, first of all, that

\footnotetext{
${ }^{4}$ The arguments below apply independently to the real and the imaginary parts of the complex-valued $\tau_{M N}$ that enters in (2.39).

${ }^{5}$ This becomes even more evident if one denotes the pair of indices $(M N)$ as $a$ and the pair $(L R)$ as $b$. Then the action of $\Lambda$ on $\tau$ takes the form $\tau^{\prime a}=\Lambda^{a b} \tau_{b}$.

${ }^{6}$ The condition $\tau_{M N}^{\prime} k^{N}=0$ gives $D-1$ constraints. This is due to the fact that $k^{2}=0$, which allows an arbitrariness in $\tau^{\prime}$ of the form $\alpha k_{M} k_{N}$.
} 
the change of the momentum of the system carried away by gravitational waves during the collision can be computed by integrating the flux of momentum through $B_{t}$ and also over time

$$
\Delta P^{M}=\int_{-\infty}^{\infty} d t \int_{B_{t}} S^{M N}(h) d^{D-2} B_{N}
$$

Note that the hypersurface $B_{t}$ is the product $S^{2} \times T^{d}$ and the corresponding measure is $d^{D-2} B_{N}=r^{2} d \Omega_{2} d^{d} \mathbf{y}$, with $r$ the radial coordinate in $R^{3}$. Thus, using Gauss' theorem one may write:

$$
\int_{W} \partial_{N} S^{M N} d^{4} x d^{d} \mathbf{y}=\int_{\Sigma_{\infty}} S^{M 0} d^{3} x d^{d} \mathbf{y}-\int_{\Sigma_{-\infty}} S^{M 0} d^{3} x d^{d} \mathbf{y}+\lim _{r \rightarrow \infty} \int_{-\infty}^{\infty} d t \int_{T^{d}} d^{d} \mathbf{y} \oint S^{M r} r^{2} d \Omega_{2} .
$$

Given that for $t= \pm \infty$ the metric is flat, ${ }^{7}$ the contribution of the integrals over $\Sigma_{ \pm \infty}$ is zero. Taking, in addition, into account the asymptotic behavior of ${ }^{2} h$ and its derivatives (see appendix D), one concludes that (2.44) takes the form:

$$
\Delta P^{M}=\int_{W} \partial_{N} S^{M N}\left({ }^{2} h\right) d^{D} x .
$$

Finally, repeating the same steps and arguments as between equations (2.30) and (2.32) and taking into account the asymptotic behavior relevant to the case at hand, one ends up with the same expression as in the $M_{1, D-1}$ case for the emitted momentum

$$
\Delta P_{M}=\frac{1}{2} \int_{W}{ }^{2} h_{P Q, M} \square^{2} \psi^{P Q} d^{D} x .
$$

In terms of the Fourier transformed quantities defined by

$$
\begin{aligned}
& h^{M N}(x, y)=\frac{1}{(2 \pi)^{4} V} \sum_{n} \int h_{(n)}^{M N}\left(k^{\mu}\right) e^{-i k_{\mu} x^{\mu}+i n_{i} y^{i} / R} d^{4} k \\
& \tau^{M N}(x, y)=\frac{1}{(2 \pi)^{4} V} \sum_{n} \int \tau_{(n)}^{M N}\left(k^{\mu}\right) e^{-i k_{\mu} x^{\mu}+i n_{i} y^{i} / R} d^{4} k
\end{aligned}
$$

( $V$ is the volume of the $d$-dimensional torus) the emitted momentum in the transverse to the brane directions takes the form

$$
\Delta P^{i}=\int_{\mathbb{R}^{3} \times T^{d}} \tau^{i 0} d^{3} x d^{d} \mathbf{y} \sim \int d k^{0} d k^{\prime 0} \sum_{n \in \mathbb{Z}^{d}} \frac{\mathrm{e}^{i\left(k^{0}-k^{\prime 0}\right) t} k^{0}}{\left[\left(k^{0}\right)^{2}-\mathbf{k}^{2}-k_{T}^{2}\right]\left[\left(k^{\prime 0}\right)^{2}-\mathbf{k}^{2}-k_{T}^{2}\right]} n^{i},
$$

where $\mathbf{k}$ is a wave 3 -vector on the brane and $k_{T}^{2} \equiv n^{2} / R^{2} . \Delta P^{i}$ vanishes since the integrand is odd under parity $\mathbf{n} \rightarrow-\mathbf{n}$. A similar symmetry argument leads to the conclusion that all components of $\Delta P^{M}$ perpendicular to the scattering plane vanish as well.

\footnotetext{
${ }^{7}$ In this calculation we ignore the self-action of fields upon the particles, but even if we did not, the change of (infinite) self-field momenta would be zero.
} 


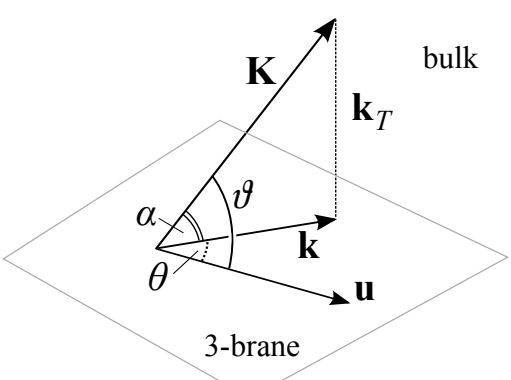

(a)

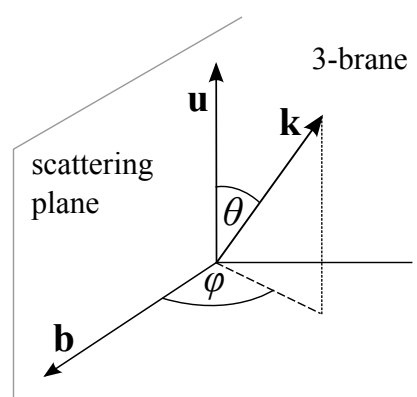

(b)

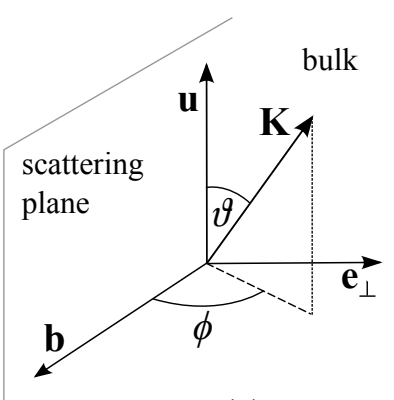

(c)

Figure 2. The angles in lab frame used in the text.

Finally, the emitted energy $\left(E \equiv \Delta P_{0}\right)$ is

$$
E=\left.\frac{\varkappa_{D}^{2}}{32 \pi^{3} V} \sum_{n \in \mathbb{Z}^{d}} \int_{0}^{\infty} \mathbf{k}^{2} d|\mathbf{k}| \int_{S^{2}} d \Omega \tau_{S N}(k) \tau_{L R}^{*}(k) \Lambda^{S N L R}\right|_{k^{0}=\sqrt{\mathbf{k}^{2}+k_{T}^{2}}},
$$

where $\tau_{M N}(k)$ is the four-dimensional Fourier-transform of the total source evaluated at $k^{0}=\sqrt{\mathbf{k}^{2}+k_{T}^{2}}$.

The wave vector of the emitted gravitational wave is a genuine $D$-dimensional vector with quantized transverse components. We denote

$$
k^{M}=\left(k^{\mu}, k_{T}^{i}\right)=\left(k^{0}, \mathbf{K}\right), \quad k_{T}^{i}=\frac{n^{i}}{R}, \quad n^{i} \in \mathbb{Z} .
$$

The notation and conventions for the angles used in this paper is shown in figure 2 .

For the brane four-vector $k^{\mu}$ we use the following parametrization ensuring zero square of $k^{M}$ :

$$
k^{\mu}=\left(\sqrt{\varpi^{2}+k_{T}^{2}}, \varpi \sin \theta \cos \varphi, \varpi \sin \theta \sin \varphi, \varpi \cos \theta\right) .
$$

Finally, using (2.42) the formula for the emitted energy becomes

$$
E=\left.\frac{\varkappa_{D}^{2}}{32 \pi^{3} V} \sum_{\mathcal{P}} \sum_{n \in \mathbb{Z}^{d}} \int_{0}^{\infty} \varpi^{2} d \varpi \int_{S^{2}} d \Omega\left|\tau_{S N}(k) \varepsilon_{\mathcal{P}}^{S N}\right|^{2}\right|_{k^{0}=\sqrt{\varpi^{2}+k_{T}^{2}}} .
$$

\subsection{Graviton polarizations}

The computation of the emitted energy can be simplified considerably if one chooses appropriately the orthonormal basis of the $D(D-3) / 2$ graviton polarization tensors $\left\{\varepsilon_{M N}^{\mathcal{P}}\right\}$ that appear in (2.43) and (2.53). The construction of such a convenient basis is presented here.

- The first step towards their construction is to define $D-2$ space-like unit vectors orthogonal to $k$ and $u^{\prime}$ and among themselves. The first $D-4$ vectors $e_{\alpha}^{M}, \alpha=$ $3, \ldots, D-2$ we choose to be orthogonal to the collision three-dimensional subspace 
spanned by the vectors $u^{M}, u^{M}, b^{M}$ which lie on the brane and together with $k$ fully characterize the kinematics of the problem under study. Thus, they are taken to satisfy the orthogonality properties

$$
e_{\alpha}^{M} e_{\beta M}=-\delta_{\alpha \beta}, \quad\left(e_{\alpha} k\right)=\left(e_{\alpha} u^{\prime}\right)=\left(e_{\alpha} u\right)=\left(e_{\alpha} b\right)=0 .
$$

As mentioned above, in the lab frame $u^{\prime M}=(1,0, \ldots, 0)$. Also, with no loss of generality the vectors $e_{\alpha}$ can be chosen to have vanishing components along the brane.

Consider next that the brane Cartesian coordinate frame $\{t, x, y, z\}$ together with the above $D-4$ space-like unit vectors $e_{\alpha}^{M}$ form a $D$-dimensional right-handed basis $\left\{t, x, y, z, e_{3}, \ldots, e_{D-2}\right\}$ and choose the $D$-dimensional Levi-Civita symbol so that $\epsilon^{0 x y z 3 \ldots(D-2)}=1$. The two remaining space-like vectors $e_{1}$ and $e_{2}$ are defined as follows: $e_{2}^{M}$ is chosen orthogonal to both $u^{M}, u^{\prime M}$, namely

$$
e_{2}^{M}=N^{-1} \epsilon^{M M_{1} M_{2} \ldots M_{D-1}} u_{M_{1}} u_{M_{2}}^{\prime} k_{M_{3}} e_{3 M_{4}} \ldots e_{(D-2) M_{D-1}},
$$

where $N$ is the normalization factor which can be obtained by squaring the above expression. Specifically, making use of the relation $k^{2}=0$ one obtains

$$
N^{2}=-\left[\left(u^{\prime} k\right) u-(u k) u^{\prime}\right]^{2} .
$$

According to this definition

$$
\left(e_{2} u\right)=\left(e_{2} u^{\prime}\right)=\left(e_{2} k\right)=\left(e_{2} e_{\alpha}\right)=0, \quad \alpha=3,4, \ldots, D-2 .
$$

Finally, the remaining vector $e_{1}$ is chosen orthogonal to $u^{\prime}$ and to all previously constructed polarization vectors. It is given by

$$
e_{1}^{M}=N^{-1}\left[(k u) u^{\prime M}-\left(k u^{\prime}\right) u^{M}+\left(\left(u u^{\prime}\right)-\frac{(k u)}{\left(k u^{\prime}\right)}\right) k^{M}\right]
$$

with the same normalization factor. Note that $e_{2}^{M}$ is confined on the brane, while $e_{1}^{M}$ has both brane and bulk components due to $k^{M}$.

- The next step is to use the above basis of orthonormal polarization vectors to build the $N_{\mathcal{P}}=D(D-3) / 2$ traceless mutually orthogonal polarization tensors $\varepsilon_{\mathcal{P}}^{M N}$, i.e. satisfying:

$$
\varepsilon_{\mathcal{P}}^{M N} \eta_{M N}=0 \text { and } \varepsilon_{\mathcal{P}}^{M N} \varepsilon_{\mathcal{P}^{\prime} M N}=\delta_{\mathcal{P} \mathcal{P}^{\prime}} .
$$

First, we build from $e_{1}$ and $e_{2}$ two tensors, the ones familiar from four-dimensional gravity:

$$
\varepsilon_{(\mathrm{I})}^{M N}=\frac{e_{1}^{M} e_{1}^{N}-e_{2}^{M} e_{2}^{N}}{\sqrt{2}}, \quad \varepsilon_{(\mathrm{II})}^{M N}=\frac{e_{1}^{M} e_{2}^{N}+e_{2}^{M} e_{1}^{N}}{\sqrt{2}},
$$

or, equivalently, the corresponding chiral ones

$$
\varepsilon_{ \pm}^{M N}=\frac{\varepsilon_{(\mathrm{I})}^{M N} \pm i \varepsilon_{(\mathrm{II})}^{M N}}{\sqrt{2}}=e_{ \pm}^{M} e_{ \pm}^{N}, \quad e_{ \pm}^{M}=\frac{e_{1}^{M} \pm i e_{2}^{M}}{\sqrt{2}}
$$


The remaining set of tensors is taken to have the same general form as the type-I and type-II above, namely to have (up to coefficients) the form of a sum of same index binomials $e_{i}^{M} e_{i}^{N}$ for the first kind, and different index binomials $e_{i}^{(M} e_{j}^{N)}$ for tensors of the 2nd type. Thus, the third tensor involving $e_{1}, e_{2}$, contains the diagonal sum of the $D-4$ vectors $e_{\alpha}^{M}$

$$
\varepsilon_{(\mathrm{III})}^{M N}=\sqrt{\frac{2(D-4)}{D-2}}\left[\frac{1}{D-4} \sum_{\alpha=3}^{D-2} e_{\alpha}^{M} e_{\alpha}^{N}-\frac{e_{1}^{M} e_{1}^{N}+e_{2}^{M} e_{2}^{N}}{2}\right] .
$$

For $D>4$ one can build the larger set of $(D-2)(D-3) / 2$ tensors similar to $\varepsilon_{(\mathrm{II})}$, starting with the full set of unit vectors $e_{1}, e_{2}, e_{\alpha}$, namely

$$
\varepsilon_{(i j)}^{M N}=\frac{e_{i}^{M} e_{j}^{N}+e_{j}^{M} e_{i}^{N}}{\sqrt{2}}, \quad i<j, \quad j=1,2, \ldots, D-2 .
$$

Finally, the remaining $D-5$ polarization tensors $\varepsilon_{\alpha}$ are obtained from the linearly independent tensors

$$
f_{\alpha}^{M N}=\frac{1}{\sqrt{(D-5)(D-4)}}\left[(D-5) e_{\alpha}^{M} e_{\alpha}^{N}-\sum_{\beta \neq \alpha} e_{\beta}^{M} e_{\beta}^{N}\right], \quad \alpha=3, \ldots, D-3 .
$$

built from $e_{\alpha}$, after ortho-normalization to satisfy the conditions $(2.59) .{ }^{8}$ For $D \leqslant 5$ there are no such polarizations.

- Finally, one can verify that $\Lambda^{M N L R}$ can be written in terms of these $D(D-3) / 2$ polarization tensors $\varepsilon_{\mathcal{P}}, \mathcal{P}=\mathrm{I}, \mathrm{II}, \mathrm{III},(i j), \alpha$, in the form

$$
\Lambda^{M N L R}=\sum_{\mathcal{P}}^{N_{\mathcal{P}}} \varepsilon_{\mathcal{P}}^{M N} \varepsilon_{\mathcal{P}}^{L R} .
$$

As advertised, the advantage of using the above special choice of polarization tensors in (2.43) and (2.53) is now evident. The indices of the energy-momentum tensor $\tau_{M N}$ are carried by the four independent vectors $u, u^{\prime}, b, k$, of which the first three belong to the brane, while $k^{M}$ is multidimensional. These four entirely characterize the bremsstrahlung process. A consequence of the fact that the vectors $e_{\alpha}$ are by construction orthogonal to those, is that only the three polarizations, namely with $\mathcal{P}= \pm$ and III contribute to (2.43) and (2.53). Moreover, the $e_{\alpha}$ terms in $e_{\mathrm{III}}$ can also be dropped and the latter simplifies to the "effective" polarization:

$$
\varepsilon_{(\mathrm{III})}^{M N}=-\sqrt{\frac{D-4}{2(D-2)}}\left[e_{1}^{M} e_{1}^{N}+e_{2}^{M} e_{2}^{N}\right]=-\sqrt{\frac{2(D-4)}{D-2}} e_{+}^{(M} e_{-}^{N)} .
$$

\footnotetext{
${ }^{8}$ One could a priori take $\alpha=3,4, \ldots, D-2$ for the label of the polarizations $f_{\alpha}$. However, they are not independent, since as one can easily verify $\sum_{\alpha=3}^{D-2} f_{\alpha}^{M N}=0$.
} 
Incidentally, notice that this effective $\varepsilon_{(\mathrm{III})}^{M N}$ vanishes, as it should, in the special case of four dimensions.

Having chosen the vectors $e_{1}^{M}$ and $e_{2}^{M}$ to be orthogonal to $k^{M}$ and $u^{\prime M}$, only the scalar products of $e_{1}^{M}$ and $e_{2}^{M}$ with $u^{M}$ and $b^{M}$ will enter in the computation of the radiated energy momentum. According to (2.57), $\left(e_{2} u\right)$ vanishes. The remaining non-vanishing products $\left(e_{1} b\right),\left(e_{1} u\right)$ and $\left(e_{2} b\right)$ are given in the appendix A.2.

\section{The gravitational radiation source}

To linear order the metric perturbation is just the sum $h_{M N}+h_{M N}^{\prime}$ of the separate contributions of the two particles. They are for each particle the solution of (2.18) with the corresponding zeroth order energy momentum tensor (2.17) for its source. In Fourier space they are given by ${ }^{9}$

$$
\begin{array}{ll}
h_{M N}=\sum_{l \in \mathbb{Z}^{d}} h_{M N}^{l}(q), & h_{M N}^{l}(q)=\frac{2 \pi \varkappa_{D} m}{q^{2}-q_{T}^{2}} \mathrm{e}^{i q b} \delta(q u)\left(u_{M} u_{N}-\frac{1}{d+2} \eta_{M N}\right) ; \\
h_{M N}^{\prime}=\sum_{l \in \mathbb{Z}^{d}} h^{\prime l}{ }_{M N}(q), & h^{\prime l}{ }_{M N}(q)=\frac{2 \pi \varkappa_{D} m^{\prime}}{q^{2}-q_{T}^{2}} \delta\left(q u^{\prime}\right)\left(u_{M}^{\prime} u_{N}^{\prime}-\frac{1}{d+2} \eta_{M N}\right),
\end{array}
$$

where $q_{T}^{i}=l^{i} / R$, the transverse components of the momentum, and $q_{T}^{2}=\sum\left(q_{T}^{i}\right)^{2}$.

The deflection of the trajectory of particle $m$ due to its gravitational interaction with $m^{\prime}$ is obtained by inserting the above $h_{M N}^{\prime}$ in (2.21) and solving for ${ }^{1} z^{M}$. One obtains

$$
{ }^{1} z^{M}(s)=-\frac{i m^{\prime} \varkappa_{D}^{2}}{(2 \pi)^{3} V} \sum_{l \in \mathbb{Z}^{d}} \int d^{4} q \frac{\delta\left(q u^{\prime}\right) e^{-i q b}}{\left(q^{2}-q_{T}^{2}\right)(q u)}\left(e^{-i(q u) s}-1\right)\left[\gamma u^{\prime M}-\frac{1}{d+2} u^{M}-\frac{\gamma_{*}^{2}}{2(q u)} q^{M}\right],
$$

where $\gamma_{*}^{2} \equiv \gamma^{2}-1 /(d+2)$. Similarly for ${ }^{1} z^{\prime M}$. As expected the trajectory deviation in the transverse directions

$$
\delta y^{i} \sim \sum_{l \in \mathbb{Z}^{d}} \int d^{4} q \frac{q_{T}^{i}}{q^{2}-q_{T}^{2}} \frac{\delta\left(q u^{\prime}\right)}{(q u)^{2}} e^{-i q b}\left(e^{-i(q u) s}-1\right)
$$

vanishes, since the summand is odd under parity $\left(q_{T}^{i} \rightarrow-q_{T}^{i}\right)$, and as expected the particles do not leave the scattering plane.

\subsection{The local part ${ }^{1} T_{M N}+{ }^{1} T_{M N}^{\prime}$ of the source}

The contributions of the particle trajectories to the gravitational radiation source is given in (2.25). They can equivalently be written in the form

$$
\begin{gathered}
{ }^{1} T_{M N}(k)=e^{i k z(0)} m \int d \tau e^{i(k u) \tau}\left[i\left(k^{1} z\right) u_{M} u_{N}+2 u_{(M}{ }^{1} \dot{z}_{N)}-\right. \\
\left.-\varkappa_{D} \frac{h^{\prime}}{2} u_{M} u_{N}+2 \varkappa_{D} u^{S} h_{S(M}^{\prime} u_{N)}\right],
\end{gathered}
$$

\footnotetext{
${ }^{9}$ It is worth noting that in four dimensions and in the center of mass frame the limit of $h_{M N}$ for $m \rightarrow 0$ and $\gamma \rightarrow \infty$ with $\mathcal{E}=m \gamma$ fixed, is the Aichelburg-Sexl metric [113].
} 
and

$$
\begin{aligned}
&{ }^{1} T_{M N}^{\prime}(k)=e^{i k z^{\prime}(0)} m^{\prime} \int d \tau e^{i\left(k u^{\prime}\right) \tau}\left[i\left(k^{1} z^{\prime}\right) u_{M}^{\prime} u_{N}^{\prime}+2 u_{(M}^{\prime}{ }^{1} \dot{z}_{N)}^{\prime}-\right. \\
&\left.-\varkappa_{D} \frac{h}{2} u_{M}^{\prime} u_{N}^{\prime}+2 \varkappa_{D} u_{S}^{\prime} h_{(M}^{S} u_{N)}^{\prime}\right],
\end{aligned}
$$

respectively. With the specific choice of polarization tensors made above, the projection of ${ }^{1} T_{M N}^{\prime}(k)$ on any of them vanishes. Then, substituting the $h^{\prime}$ and ${ }^{1} z$ given in $(3.2)$ and (3.3), one obtains: ${ }^{10}$

$$
{ }^{1} T_{M N}(k)=\frac{m m^{\prime} \varkappa^{2}}{(2 \pi)^{2} V} e^{i k b} \sum_{l \in \mathbb{Z}^{d}}\left[\left(\gamma \frac{k u^{\prime}}{k u} I^{l}-\frac{\gamma_{*}^{2}}{2(k u)^{2}}\left(k \cdot I^{l}\right)\right) u_{M} u_{N}+\frac{\gamma_{*}^{2}}{(k u)} u_{(M} I_{N)}^{l}\right],
$$

in terms of the integrals $I^{l}$ and $I_{M}^{l}$, defined by

$$
I^{l}=\int \frac{\delta\left(p u^{\prime}\right) \delta(k u-p u) \mathrm{e}^{-i(p b)}}{p^{2}-p_{T}^{2}} d^{4} p, \quad I_{M}^{l}=\int \frac{\delta\left(p u^{\prime}\right) \delta(k u-p u) \mathrm{e}^{-i(p b)}}{p^{2}-p_{T}^{2}} p_{M} d^{4} p,
$$

with $p_{T}^{i}=l^{i} / R$. These were computed in [120] and expressed in terms of Macdonald functions

$$
I^{l}=-\frac{2 \pi}{\gamma v} K_{0}\left(z_{l}\right), \quad I_{M}^{l}=-\frac{2 \pi}{\gamma v b^{2}}\left(b z K_{0}\left(z_{l}\right) \frac{\gamma u_{M}^{\prime}-u_{M}}{\gamma v}+i \hat{K}_{1}\left(z_{l}\right) b_{M}\right),
$$

with argument

$$
z_{l} \equiv\left(z^{2}+p_{T}^{2} b^{2}\right)^{1 / 2}, \quad z \equiv \frac{(k u) b}{\gamma v},
$$

while the hatted Macdonald functions are defined by $\hat{K}_{\nu}(x) \equiv x^{\nu} K_{\nu}(x)$.

Substituting back into (3.7) one obtains

$$
\begin{aligned}
{ }^{1} T_{M N}(k)=-\frac{m m^{\prime} \varkappa_{D}^{2}}{4 \pi V} \frac{e^{i k b}}{\gamma v^{3}} \sum_{l \in \mathbb{Z}^{d}}[ & -i \Gamma \frac{\hat{K}_{1}\left(z_{l}\right)}{z^{2}} \sigma_{M N}-2 \Gamma \gamma K_{0}\left(z_{l}\right) u_{(M} u_{N)}^{\prime}+ \\
& \left.+\left(\left(2 v^{2}-\Gamma\right) \gamma \frac{z^{\prime}}{z}-\Gamma\right) K_{0}\left(z_{l}\right) u_{M} u_{N}\right]
\end{aligned}
$$

where

$$
\sigma_{M N} \equiv(k b) u_{M} u_{N}-2(k u) u_{(M} b_{N)} \quad \text { and } \quad \Gamma \equiv \frac{\gamma_{*}^{2}}{\gamma^{2}}=1-\frac{1}{(d+2) \gamma^{2}} .
$$

Finally, omitting the terms longitudinal in $u^{\prime}$, since they give zero when contracted with any polarization tensor, the above expression simplifies to ${ }^{11}$

$$
{ }^{1} T_{M N}(k)=-\frac{m m^{\prime} \varkappa_{D}^{2}}{4 \pi V} \frac{e^{i k b}}{\gamma v^{3}} \sum_{l \in \mathbb{Z}^{d}}\left[-i \Gamma \frac{\hat{K}_{1}\left(z_{l}\right)}{z^{2}} \sigma_{M N}+\left(\left(2 v^{2}-\Gamma\right) \gamma \frac{z^{\prime}}{z}-\Gamma\right) K_{0}\left(z_{l}\right) u_{M} u_{N}\right] .
$$

\footnotetext{
${ }^{10}$ Note, incidentally, that as expected the part of ${ }^{1} z_{M}$ which corresponds to uniform motion, i.e. the term -1 in the first parenthesis, does not contribute to (3.7).

${ }^{11}$ In what follows we will omit from the radiation source all terms, which give zero when contracted with all polarization tensors, since they do not contribute to the emitted energy-momentum.
} 
As in previous analogous cases [106, 120], the exponential fall-off of the Macdonald functions for large values of the argument $z_{l}$ leads to an effective natural cut-off $N_{\text {int }}$ on the number of interaction modes $l$ in the sum. One can estimate the radius $l_{\text {int }}$ of the sphere in the $\left\{l^{i}\right\}$ space, beyond which the modes can be neglected, by setting

$$
\left(l_{\text {int }} / R\right)^{2} b^{2} \sim 1
$$

from which the number of contributing interaction modes is obtained

$$
N_{\text {int }} \sim\left(\frac{R}{b}\right)^{d} \sim \frac{V}{b^{d}}
$$

For $b \ll R$, which is the case of interest here, one has $N_{\text {int }} \gg 1$, and the mode-summation can be converted to integration using [120] $(Z>0)$

$$
\frac{1}{V} \sum_{l} \hat{K}_{\lambda}\left(\sqrt{Z^{2}+p_{T}^{2} b^{2}}\right) \simeq \frac{1}{(2 \pi)^{d / 2} b^{d}} \hat{K}_{\lambda+d / 2}(Z)
$$

to end up with

$$
{ }^{1} T_{M N}(k)=-\frac{\lambda e^{i(k b)}}{\gamma v^{3}}\left[-i \Gamma \frac{\hat{K}_{d / 2+1}(z)}{z^{2}} \sigma_{M N}+\left(\left(2 v^{2}-\Gamma\right) \gamma \frac{z^{\prime}}{z}-\Gamma\right) \hat{K}_{d / 2}(z) u_{M} u_{N}\right],
$$

where

$$
\lambda \equiv \frac{\varkappa_{D}^{2} m^{\prime} m}{2(2 \pi)^{d / 2+1} b^{d}} .
$$

Angular and frequency characteristics. The local radiation source (3.17) above is expressed solely in terms of Macdonald functions with argument $z$. As has been demonstrated in analogous instances before [106, 120], the exponential fall-off of these functions implies an effective cut-off of $\mathcal{O}(1)$ in the angle and frequency dependent variable $z$, which eventually constrains the angular and frequency distribution of the radiation. Below, it will be shown that a similar situation arises as well with the non-local amplitude $S_{M N}$.

\subsection{The non-local part $S_{M N}$ of the source}

The stress tensor $S_{M N}$ is defined in (A.5) and calculated in coordinate representation in (2.9). Again, since its trace does not contribute to the emitted momentum, one may restrict himself to its traceless part. Write, as explained above, $h_{M N}+h_{M N}^{\prime}$ for the leading metric perturbation and substitute in $S_{M N}$, while omitting self-action terms, to obtain for its $n$-th mode (in analogy to (2.48))

$$
\begin{aligned}
& S_{M N}^{(n)}\left(x^{\mu}\right)=\frac{1}{V} \sum_{l}\left[h_{M}^{(n-l) A, B}\left({h_{N B, A}^{\prime(l)}}^{(l)}{h^{\prime}}_{N A, B}^{(l)}\right)-\frac{1}{2} h_{A B, N}^{(n-l)}{h^{(l) A B}}_{, M}-\frac{1}{2} h_{M N}^{(n-l)} \square h^{(l)}+\right. \\
& \left.+\left(h_{M A, N B}^{(n-l)}+h_{N A, M B}^{(n-l)}-h_{A B, M N}^{(n-l)}-h_{M N, A B}^{(n-l)}\right) h^{(l) A B}\right]+\left\{h \longleftrightarrow h^{\prime}\right\},
\end{aligned}
$$


and with $S_{M N}^{(n)}\left(x^{\mu}\right)$ the four-dimensional Fourier-transform of $S_{M N}^{(n)}\left(k^{\mu}\right)$. Upon substitution of the solutions (3.1) we have in momentum representation ${ }^{12}$

$$
S_{M N}^{(n)}(k)=\frac{m m^{\prime} \varkappa_{D}^{2} e^{i(k b)}}{4 \pi^{2}}\left[u_{M} u_{N}\left(k u^{\prime}\right)^{2} J^{n}(k)+2 \gamma\left(k u^{\prime}\right) u_{(M} J_{N)}^{n}(k)+\gamma_{*}^{2} J_{M N}^{n}(k)\right] .
$$

The non-vanishing components of the scalar, vector and tensor integrals that appear in (3.20), namely

$$
\begin{gathered}
J^{n}(k) \equiv \frac{1}{V} \sum_{l} \int d^{4} p \frac{\delta\left(p u^{\prime}\right) \delta(k u-p u) e^{-i(p b)}}{\left(p^{2}-p_{T}^{2}\right)\left[(k-p)^{2}-\left(k_{T}^{i}-p_{T}^{i}\right)^{2}\right]}, \quad p^{M}=\left(p^{\mu}, p_{T}^{i}\right), \\
J_{M}^{n}(k) \equiv \frac{1}{V} \sum_{l} \int d^{4} p \frac{\delta\left(p u^{\prime}\right) \delta(k u-p u) e^{-i(p b)}}{\left(p^{2}-p_{T}^{2}\right)\left[(k-p)^{2}-\left(k_{T}^{i}-p_{T}^{i}\right)^{2}\right]} p_{M},
\end{gathered}
$$$$
p^{2} \equiv p_{\mu} p^{\nu}
$$

and $^{13}$

$$
J_{M N}^{n}(k) \equiv \frac{1}{V} \sum_{l} \int d^{4} p \frac{\delta\left(p u^{\prime}\right) \delta(k u-p u) e^{-i(p b)}}{\left(p^{2}-p_{T}^{2}\right)\left[(k-p)^{2}-\left(k_{T}^{i}-p_{T}^{i}\right)^{2}\right]} p_{M} p_{N},
$$

are computed in appendix B. Using (B.8), (B.15) and (B.17) one obtains ${ }^{14}$

$$
\begin{aligned}
& S_{M N}(k)=\frac{\lambda e^{i(k b)}}{v} \int_{0}^{1} \mathrm{e}^{-i(k b) x}\left[b^{2}\left(\Gamma \gamma \tilde{N}_{M} \tilde{N}_{N}+\frac{1}{\gamma}\left(k u^{\prime}\right)^{2} u_{M} u_{N}+2\left(k u^{\prime}\right) u_{(M} \tilde{N}_{N)}\right) \hat{K}_{d / 2-1}\left(\zeta_{n}\right)+\right. \\
& \left.+\left(2 i\left(k u^{\prime}\right) u_{(M} b_{N)}+\frac{\Gamma}{\gamma v^{2}}\left[2 i \gamma^{2} v^{2} b_{(M} \tilde{N}_{N)}-u_{M} u_{N}\right]\right) \hat{K}_{d / 2}\left(\zeta_{n}\right)+\frac{b_{M} b_{N}}{b^{2}} \gamma \Gamma \hat{K}_{d / 2+1}\left(\zeta_{n}\right)\right] d x
\end{aligned}
$$

with $\zeta_{n}$ given in (B.7). Upon substitution of $\tilde{N}_{M} \equiv-\left[(1-x)(k u)+\gamma x\left(k u^{\prime}\right)\right] u_{M} /\left(\gamma^{2} v^{2}\right)$, one ends-up with the fully expanded expression

$$
S_{M N}(k)=\frac{\lambda e^{i(k b)}}{v} \int_{0}^{1} \mathrm{e}^{-i(k b) x} Y_{M N}(k, x) d x
$$

with

$$
\begin{aligned}
& Y_{M N}(k, x)=\frac{b_{M} b_{N}}{b^{2}} \gamma \Gamma \hat{K}_{d / 2+1}\left(\zeta_{n}\right)+ \\
& {\left[2 i u_{(M} b_{N)}\left(\left(k u^{\prime}\right)\left(1-\frac{\Gamma x}{v^{2}}\right)-\frac{\Gamma}{\gamma v^{2}}(1-x)(k u)\right)-\frac{\Gamma}{\gamma v^{2}} u_{M} u_{N}\right] \hat{K}_{d / 2}\left(\zeta_{n}\right)+} \\
& {\left[\left(k u^{\prime}\right)^{2}\left(1-\frac{2 x}{v^{2}}+\frac{\Gamma x^{2}}{v^{4}}\right)-2 \frac{(k u)\left(k u^{\prime}\right)(1-x)}{\gamma v^{2}}\left(1-\frac{\Gamma x}{v^{2}}\right)+\frac{\Gamma(k u)^{2}(1-x)^{2}}{\gamma^{2} v^{4}}\right] \frac{b^{2}}{\gamma} u_{M} u_{N} \hat{K}_{d / 2-1}\left(\zeta_{n}\right)}
\end{aligned}
$$

\footnotetext{
${ }^{12}$ All terms, which upon contraction with the polarization tensors give zero, have been omitted from $S_{M N}^{(n)}(k)$.

${ }^{13}$ Note that the bulk components $J_{i}^{n}$ and $J_{i M}^{n}$ vanish by parity only if $k_{T}^{i}=0$.

${ }^{14}$ We passed again from summation into integration. Also, to simplify notation a bit, the index $(n)$, labeling the number of emission KK-mode, is suppressed.
} 
Like the local source (3.17), the non-local $S_{M N}$ in its effective form (3.25) is also confined on the brane. Furthermore, $S_{M N}$ is a linear combination of terms proportional to integrals of the form

$$
J_{(\sigma, \tau)} \equiv \Lambda_{d} \int_{0}^{1} x^{\sigma} e^{-i(k b) x} \hat{K}_{d / 2+\tau}\left(\zeta_{n}\right) d x
$$

(with $\sigma=0,1,2$ ), defined in (B.19) and studied in appendix B. ${ }^{15}$ In [106] it was shown that these integrals can be written as a sum of two terms. One, is expressed in terms of hatted Macdonald functions of only the variable $z$ and the other hatted Macdonalds of another variable $z^{\prime}$. So, one writes in an obvious notation

$$
J_{(\sigma, \tau)}=J_{(\sigma, \tau)}^{[z]}+J_{(\sigma, \tau)}^{\left[z^{\prime}\right]}, \quad z \equiv \frac{(k u) b}{\gamma v}, \quad z^{\prime} \equiv \frac{\left(k u^{\prime}\right) b}{\gamma v} .
$$

The total radiation amplitude is thus naturally written as a sum of two terms. Both expressed in terms of Macdonald functions, but with argument $z$ the first and $z^{\prime}$ the second. As explained in [106] the characteristics of the Macdonald functions translate eventually into angular and frequency distribution properties of the emitted radiation.

\subsection{Destructive interference of the beamed high-frequency components}

As explained in [105] and [106], if the radiation source consisted of the local part $T_{M N}$ alone, which is expressed in terms of Macdonalds with $\operatorname{argument} z$, the emitted radiation would be mainly $z$-type, i.e. beamed $\vartheta \sim \gamma^{-1}$, with high characteristic frequency $\omega \sim \gamma^{2} / b$. Now, it will be shown, exactly like in scalar radiation discussed in [106], that in the sum $T_{M N}+S_{M N}$ the two leading powers of $\gamma$ in the ultra-relativistic expansions of $T_{M N}$ and $S_{M N}$ exactly cancel and the total amplitude is suppressed, compared to each one separately, by two powers of $\gamma$. This cancelation is a consequence of the equivalence principle: geodesics of the ultrarelativistic particles and of the emitted massless quanta stay close together and enhance the formation length of radiation $[114,115]$.

We will not repeat here the derivation presented in [106]. Instead, we will just highlight the main steps of the derivation in the present context.

Step 1: for $z$-type radiation, one has $z \sim \mathcal{O}(1)$ and $z^{\prime} \sim \mathcal{O}(\gamma)$. Thus, the contribution of $J^{\left[z^{\prime}\right]}$, which contains only Macdonalds of $z^{\prime}$ are exponentially suppressed and can be neglected. Thus, the dominant contribution to $z$-type radiation is due to $J^{[z]}$.

Step 2: the integrals appearing in $S_{M N}$ with $\sigma=1,2$ are suppressed compared to the one with $\sigma=0$. Consider for instance $J_{(1, \tau)}$. Using (B.22), (B.32) and (B.34) from appendix $\mathrm{B}$ one can write for its leading terms

$J_{(1, \tau)}^{[z]}=\frac{\Lambda_{d}}{\xi^{2}}\left(-\sin ^{2} \vartheta \hat{K}_{d / 2+\tau+1}(z)-\frac{(d+2 \tau+3) \beta^{2}}{a^{4} \xi^{2}} \hat{K}_{d / 2+\tau+2}(z)+\frac{\beta^{2}}{a^{4} \xi^{2}} \hat{K}_{d / 2+\tau+3}(z)\right)+\ldots$

\footnotetext{
${ }^{15}$ Note that the coefficient $\Lambda_{d}$, which appeared naturally in the definition (B.19) of appendix B, is kept here also, in order to avoid the introduction of more symbols. Also, note that $J_{(\sigma, \tau)}$ are clearly functions of $k$ and $n$, which are suppressed in order to simplify notation a bit.
} 
with $a, \beta$ and $\xi$ defined in appendices A and B. This is of order of $a^{-2} \ll 1$ compared to $J_{(0, \tau)}^{[z]}$ (given in appendix B), since in the region of interest $a=\mathcal{O}(\gamma)$.

Step 3: since the destructive interference effect has to do with the two leading terms in the local and non-local amplitudes, we focus on exactly these two. Hence, we neglect $(k u) / \gamma$ compared to $\left(k u^{\prime}\right)$, set $\Gamma=1$ and $v=1$ and omit $x$ - and $x^{2}$-terms in the integrand of (3.25). Thus, the leading part of $S_{M N}$ reads

$$
\begin{gathered}
S_{M N} \simeq \lambda e^{i(k b)} \int_{0}^{1}\left[\frac{b_{M} b_{N}}{b^{2}} \gamma \hat{K}_{d / 2+1}\left(\zeta_{n}\right)+\left(2 i u_{(M} b_{N)}\left(k u^{\prime}\right)-\frac{1}{\gamma} u_{M} u_{N}\right) \hat{K}_{d / 2}\left(\zeta_{n}\right)+\right. \\
\left.\quad+z^{\prime 2} \gamma \hat{K}_{d / 2-1}\left(\zeta_{n}\right) u_{M} u_{N}\right] \mathrm{e}^{-i(k b) x} d x
\end{gathered}
$$

Step 4: the quantities to be compared are the projections of $S_{M N}$ and $T_{M N}$ on the polarization tensors. It is easy to check, using the formulae of appendix A for the contractions of $u^{M}$ and $b^{M}$ with the polarization vectors, that at small angles $\vartheta \lesssim \gamma^{-1}$ no extra powers of $\gamma$ are introduced through such contractions. Thus, comparing directly the coefficients of $u_{M} u_{N}, u_{(M} b_{N)}$ and $b_{M} b_{N}$, one ends-up with the following expression for the leading terms of $S_{M N}$ :

$$
S_{M N} \simeq \lambda e^{i(k b)} \int_{0}^{1}\left[2 i u_{(M} b_{N)}\left(k u^{\prime}\right) \hat{K}_{d / 2}\left(\zeta_{n}\right)+z^{\prime 2} \gamma \hat{K}_{d / 2-1}\left(\zeta_{n}\right) u_{M} u_{N}\right] \mathrm{e}^{-i(k b) x} d x
$$

Step 5: using (B.10) appropriately simplified for the range of parameters of interest here, namely

$$
\int_{0}^{1} d x \mathrm{e}^{-i(k b) x} \hat{K}_{\tau-1}\left(\zeta_{n}\right) \simeq \frac{1}{\gamma z z^{\prime}} \hat{K}_{\tau}(z)-i \frac{(k b)}{\left(\gamma z z^{\prime}\right)^{2}} \hat{K}_{\tau+1}(z)+\ldots,
$$

into (3.30), one obtains

$$
S_{M N} \simeq \lambda e^{i(k b)}\left[\frac{z^{\prime}}{z} \hat{K}_{d / 2}(z) u_{M} u_{N}-i \frac{1}{\gamma z^{2}} \hat{K}_{d / 2+1}(z) \sigma_{M N}\right]
$$

plus contributions to the real and the imaginary parts suppressed by two powers of $\gamma$ compared to the terms kept in (3.31).

Step 6: making the same approximations for the local part ${ }^{1} T_{M N}$ in (3.17), one ends-up with ${ }^{1} T_{M N}=-S_{M N}$ given in (3.31) above and proves the so called destructive interference of the leading terms of local and stress amplitudes.

\subsection{The total radiation amplitude}

To summarize, the total radiation source $\tau_{M N}$ was naturally split into the sum of a function of $z$ and a function of $z^{\prime}$. The two leading terms in the expansion of the function of $z$ in powers of $\gamma$ were shown to cancel. The next step is to compute the leading terms of $\tau_{M N}$, which survive destructive interference and use them to compute the emitted radiation 
energy to leading order. The discussion follows the steps described in [106] for scalar radiation, only repeated three times because of the three relevant polarizations. That is, for each polarization one first distinguishes three characteristic regimes of angular and frequency distribution of the emitted energy, namely (a) the beamed $(\vartheta \sim 1 / \gamma)$ highfrequency $\left(\omega \sim \gamma^{2} / b\right)$ regime, (b) the beamed medium-frequency $(\omega \sim \gamma / b)$ regime, and (c) the unbeamed $\vartheta \sim 1$ medium-frequency regime, and then computes the emitted energy in each one of them.

\subsubsection{The beamed, high-frequency radiation amplitude}

In this regime $z^{\prime} \sim \mathcal{O}(\gamma)$ and the contribution of Macdonald functions of $z^{\prime}$ is exponentially suppressed.

Polarization I: projecting (3.17) and (3.25) on $\varepsilon_{\mathrm{I}}^{M N}$ and taking into account that only the $e_{1}^{M} e_{1}^{N} / \sqrt{2}$ part of $\varepsilon_{\mathrm{I}}^{M N}$ contributes, one obtains for ${ }^{1} T_{\mathrm{I}} \equiv{ }^{1} T_{M N} \varepsilon_{\mathrm{I}}^{M N}$ and $S_{\mathrm{I}} \equiv$ $S_{M N} \varepsilon_{\mathrm{I}}^{M N}$

${ }^{1} T_{\mathrm{I}}(k)=-\frac{\lambda e^{i(k b)}}{\sqrt{2} \gamma v^{3}}\left[-i \Gamma \frac{\hat{K}_{d / 2+1}(z)}{z^{2}}(k b)\left[\psi^{2} \gamma^{2}-1\right]+\left(\left(2 v^{2}-\Gamma\right) \gamma \frac{z^{\prime}}{z}-\Gamma\right) \hat{K}_{d / 2}(z) \gamma^{2} v^{2} \sin ^{2} \vartheta\right]$

and

$$
\begin{aligned}
S_{\mathrm{I}}=\frac{\lambda \gamma e^{i(k b)}}{\sqrt{2} v} \int_{0}^{1} \mathrm{e}^{-i(k b) x} d x\left\{\left(\cos ^{2} \phi \cos ^{2} \vartheta-\sin ^{2} \phi\right) \Gamma \hat{K}_{d / 2+1}\left(\zeta_{n}\right)+\right. \\
+\left[2 i v \cos \vartheta(k b)\left(1-\frac{\Gamma x}{v^{2}}-\psi \frac{\Gamma(1-x)}{v^{2}}\right)-\Gamma \sin ^{2} \vartheta\right] \hat{K}_{d / 2}\left(\zeta_{n}\right)+ \\
\left.+\left[\frac{1}{\psi^{2}}\left(1-\frac{2 x}{v^{2}}+\frac{\Gamma x^{2}}{v^{4}}\right)-2 \frac{(1-x)}{\psi v^{2}}\left(1-\frac{\Gamma x}{v^{2}}\right)+\frac{\Gamma(1-x)^{2}}{v^{4}}\right] z^{2} v^{4} \sin ^{2} \vartheta \hat{K}_{d / 2-1}\left(\zeta_{n}\right)\right\}
\end{aligned}
$$

respectively. The next step is to expand in powers of $\gamma$. For that one has to use the expansions of $\cos \vartheta, v, \Gamma$ and $\psi$ in appendix A, the fact that integrals with extra $x^{\alpha}$ in the integrand are suppressed by $\gamma^{-2 \alpha}$, and the expressions for the integrals $J_{(0, \tau)}^{[z]}$ and $J_{(1, \tau)}^{[z]}$ given in appendix B and (3.28). After a somewhat tedious but straightforward calculation one ends-up with the leading part of $\tau_{\mathrm{I}} \equiv \tau_{M N} \varepsilon_{\mathrm{I}}^{M N}$ :

$\tau_{\mathrm{I}} \simeq \frac{\lambda e^{i(k b)}}{\sqrt{2}} \frac{\sin ^{2} \vartheta}{\gamma \psi}\left[\frac{d+1}{d+2} \hat{K}_{d / 2}(z)+(d+1) \frac{\hat{K}_{d / 2+1}(z)}{z^{2}}+\left(\frac{\gamma^{2} \psi^{2} \cos 2 \phi}{\sin ^{2} \vartheta}-\sin ^{2} \phi\right) \frac{\hat{K}_{d / 2+2}(z)}{z^{2}}\right]$.

Polarization II: similarly, projection onto the second polarization tensor leads (with an analogous notation) to

$$
{ }^{1} T_{\mathrm{II}}=\frac{2 i \lambda e^{i(k b)}}{\sqrt{2} v} \frac{\hat{K}_{d / 2+1}(z)}{z} \gamma \sin \vartheta \sin \phi
$$


and

$$
\begin{aligned}
S_{\mathrm{II}}=\frac{\gamma \lambda e^{i(k b)}}{\sqrt{2} v} \int_{0}^{1} d x \mathrm{e}^{-i(k b) x}[ & \Gamma \cos \vartheta \sin 2 \phi \hat{K}_{d / 2+1}\left(\zeta_{n}\right)- \\
& \left.\quad-2 i z \sin \vartheta \sin \phi\left(\frac{v^{2}}{\psi}\left(1-\frac{\Gamma x}{v^{2}}\right)-\Gamma(1-x)\right) \hat{K}_{d / 2}\left(\zeta_{n}\right)\right] .
\end{aligned}
$$

Following the same steps as above, one obtains for the leading ultra-relativistic term in their sum

$$
\tau_{\mathrm{II}} \simeq \frac{\lambda e^{i(k b)}}{\sqrt{2}} \gamma\left(\psi+\sin ^{2} \vartheta\right) \sin 2 \phi \frac{\hat{K}_{d / 2+2}(z)}{z^{2}}
$$

Polarization III: repeating the same steps for the third polarization tensor, while taking into account that only the $b_{M} b_{N}$-binomial does not vanish upon projection on $e_{2}^{M} e_{2}^{N}$, one obtains:

$$
{ }^{1} T_{\mathrm{III}}=-\sqrt{\frac{d}{d+2}}{ }^{1} T_{\mathrm{I}}
$$

and

$$
S_{\mathrm{III}}=-\sqrt{\frac{d}{d+2}}\left[S_{\mathrm{I}}+\frac{\lambda \gamma e^{i(k b)}}{\sqrt{2}} \int_{0}^{1} 2 \sin ^{2} \phi \hat{K}_{d / 2+1}\left(\zeta_{n}\right) \mathrm{e}^{-i(k b) x} d x\right] .
$$

Combining these two and expanding, one has to leading order

$$
\begin{aligned}
& \tau_{\mathrm{III}}=-\sqrt{\frac{d}{d+2}} \frac{\lambda e^{i(k b)}}{\sqrt{2}} \frac{\sin ^{2} \vartheta}{\gamma \psi}\left[\frac{d+1}{d+2} \hat{K}_{d / 2}(z)+(d+1) \frac{\hat{K}_{d / 2+1}(z)}{z^{2}}+\right. \\
& \left.+\left(\frac{\gamma^{2} \psi^{2}}{\sin ^{2} \vartheta}-\sin ^{2} \phi\right) \frac{\hat{K}_{d / 2+2}(z)}{z^{2}}\right] .
\end{aligned}
$$

Up to the common phase factor and the factor $\lambda$ in front, all three amplitudes are real and of $\mathcal{O}(1 / \gamma)$.

\subsubsection{Wide-angle, medium-frequency radiation amplitude}

In this regime it is more convenient to consider directly the behavior of the radiation source, before one projects onto the three polarization tensors. Also, in this regime $z \sim \gamma$, and consequently the Macdonald functions of $z$ give exponentially suppressed contributions and will be neglected. Thus, the leading contribution is due to the $z^{\prime}$-dependent part of the non-local source $S_{M N}$, which we compute next.

In subsection 3.3 it was shown that for $\omega \sim \gamma^{2} / b, \vartheta \sim \gamma^{-1}$ one has $J_{(1, \tau)} \sim J_{(0, \tau)} / \gamma^{2}$. This implied that only the neighborhood of $x=0$ contributes to these integrals.

Similarly, it will be shown that in the region of interest here, namely $(\omega \sim \gamma / b, \vartheta \sim 1)$, the leading contribution to these integrals comes from the neighborhood of $x=1$. Indeed, 
consider the integral with $(1-x)$, i.e. $J_{(0, \tau)}-J_{(1, \tau)}$. Using (B.32) and subsequently (B.22) this difference takes the form

$$
J_{(0, \tau)}-J_{(1, \tau)} \simeq \frac{\Lambda_{d} \mathrm{e}^{-i(k b)}}{\xi^{2}}\left[\left(1-\frac{2}{\psi}\right) \frac{\hat{K}_{d / 2+\tau+1}\left(z^{\prime}\right)}{\gamma^{2}}+\frac{\cos ^{2} \phi}{a^{2}} \hat{K}_{d / 2+\tau+3}\left(z^{\prime}\right)+\ldots\right] .
$$

This is of order $\mathcal{O}\left(\gamma^{-2}\right)$ with respect to both $J_{(0, \tau)}$ and $J_{(1, \tau)}$. This implies that the integrals $J_{(0, \tau)}$ and $J_{(1, \tau)}$ are almost equal, and the main contribution in them comes from the domain $x=1-0$, and consequently, that integral with $(1-x)^{2}$ is even more suppressed.

Thus, it is natural to rearrange the terms of the integrand in (3.25) in powers of $1-x$ as well as in powers of $\gamma$, taking into account that each power of $1-x$ contributes a factor of $\mathcal{O}\left(1 / \gamma^{2}\right)$ to the integral. Taking in addition into account that in the region of interest here $\gamma\left(k u^{\prime}\right)$ is of the same order as $(k u)$, one obtains

$$
\begin{aligned}
\tau_{M N} & \simeq \lambda e^{i(k b)} \int_{0}^{1} d x \mathrm{e}^{-i(k b) x}\left\{\frac{b_{M} b_{N}}{b^{2}} \gamma \hat{K}_{d / 2+1}\left(\zeta_{n}\right)-\frac{1}{d+2} \frac{z^{\prime 2}}{\gamma} u_{M} u_{N} \hat{K}_{d / 2-1}\left(\zeta_{n}\right)+\right. \\
& \left.+\left[2 i u_{(M} b_{N)}\left(\left(\left(k u^{\prime}\right)-\frac{(k u)}{\gamma}\right)(1-x)-\frac{d+1}{d+2} \frac{\left(k u^{\prime}\right)}{\gamma^{2}}\right)-\frac{u_{M} u_{N}}{\gamma}\right] \hat{K}_{d / 2}\left(\zeta_{n}\right)\right\} .
\end{aligned}
$$

Using $\left(e_{1} u\right)=\gamma v \sin \vartheta,\left(e_{2} u\right)=0$ and $\left(e_{1} b\right)=\mathcal{O}(b)=\left(e_{2} b\right)$, one can simplify (3.41) and write instead

$$
\begin{aligned}
\tau_{M N} \simeq \frac{\lambda e^{i(k b)}}{\gamma} \int_{0}^{1}\left[\frac{b_{M} b_{N}}{b^{2}} \gamma^{2} \hat{K}_{d / 2+1}\left(\zeta_{n}\right)-u_{M} u_{N} \hat{K}_{d / 2}\left(\zeta_{n}\right)-\right. \\
\left.\quad-\frac{1}{d+2} z^{\prime 2} u_{M} u_{N} \hat{K}_{d / 2-1}\left(\zeta_{n}\right)\right] \mathrm{e}^{-i(k b) x} d x .
\end{aligned}
$$

It is straightforward to check that its projection onto any of the three polarization tensors gives to leading order the same result as the projection of (3.41).

From (B.22) one obtains to leading ultra-relativistic order

$$
\int_{0}^{1} \mathrm{e}^{-i(k b) x} \hat{K}_{\tau}\left(\zeta_{n}\right) d x \simeq \frac{\mathrm{e}^{-i(k b)}}{{z^{\prime}}^{2} \gamma^{2} \psi} \hat{K}_{\tau+1}\left(z^{\prime}\right)
$$

using which in (3.42), one finally ends-up with

$$
\tau_{M N} \simeq \frac{\lambda}{z^{\prime 2} \gamma^{3} \psi}\left[\frac{b_{M} b_{N}}{b^{2}} \gamma^{2} \hat{K}_{d / 2+2}\left(z^{\prime}\right)-u_{M} u_{N} \hat{K}_{d / 2+1}\left(z^{\prime}\right)-\frac{1}{d+2} z^{\prime 2} u_{M} u_{N} \hat{K}_{d / 2}\left(z^{\prime}\right)\right] .
$$

\subsubsection{The beamed, medium-frequency radiation amplitude}

Starting again from (3.17) and (3.25), one can observe that for $\vartheta \sim \gamma^{-1}$ the projection on polarization tensors does not introduce $\gamma$-factors, hence one can estimate the order in $\gamma$ directly from the coefficients of the tensor binomials $u_{M} u_{N}, b_{M} b_{N}$ and $u_{(M} b_{N)}$. In the regime of interest here one has $z \sim 1 / \gamma, z^{\prime} \sim 1$, using which one can immediately conclude that both local and stress sources, as well as their projections on the relevant polarizations are of the same order, namely $T_{M N} \sim S_{M N} \sim \tau \sim \mathcal{O}(\gamma)$ in any dimension. 


\begin{tabular}{|c|c|c|c|}
\hline & $\omega \sim 1 / b$ & $\omega \sim \gamma / b$ & $\omega \sim \gamma^{2} / b$ \\
\hline$\gamma^{-1}$ & $\begin{array}{l}\text { no destructive } \\
\text { interference } \\
\tau \sim T \gg S\end{array}$ & $\begin{array}{l}\text { no destructive interference } \\
S^{[z]} \sim T \sim S^{\left[z^{\prime}\right]} \sim \gamma\end{array}$ & $\begin{array}{l}\text { destructive interference: } \\
T \approx-S^{[z]} \\
S^{\left[z^{\prime}\right]} \sim \exp (-\gamma) \\
\tau=\mathcal{O}\left(T / \gamma^{2}\right) \sim 1 / \gamma\end{array}$ \\
\hline 1 & $\begin{array}{l}\text { no destructive } \\
\text { interference } \\
\tau \sim T \sim S\end{array}$ & $\begin{array}{l}\text { destructive interference: } \\
S^{[z]} \approx T \sim \exp (-\gamma) \\
\tau=S=S^{\left[z^{\prime}\right]} \sim \gamma^{-1}\end{array}$ & $\begin{array}{l}\text { destructive interference } \\
T \sim S \sim \tau \sim \exp (-\gamma)\end{array}$ \\
\hline
\end{tabular}

Table 1. The leading behavior of the amplitudes (projected on polarizations) in the various characteristic angular-frequency regimes. $S^{[z]}$ and $S^{\left[z^{\prime}\right]}$ stand for the contributions of integrals $J^{[z]}$ and $J^{\left[z^{\prime}\right]}$, respectively. The dependence of $\lambda$ on $\gamma$ is not included, i.e. all estimates are given in units $\lambda=1$.

\subsection{Summary}

The results of this section are summarized in table 1, which shows the leading behavior of the corresponding amplitudes after projection on the polarization tensors.

Notice that the entries in table 1 do not depend on the dimensionality $d$. Also, note that this leading dependence of the amplitudes on $\gamma$ is valid in the whole range $[0,2 \pi]$ of the azimuthal angle $\varphi$.

\section{The emitted energy}

The emitted energy, obtained from (2.43) for $M=0$ is

$$
E=\frac{\varkappa_{D}^{2}}{4(2 \pi)^{D-1}} \sum_{\mathcal{P}} \int_{0}^{\infty} \omega^{D-2} d \omega \int_{S^{D-2}} d \Omega\left|\tau_{L R}(k) \varepsilon_{\mathcal{P}}^{L R}\right|^{2} .
$$

It differs from the scalar radiation case studied in [106] by the summation over polarizations and the substitution of the scalar charge $f \rightarrow \varkappa_{D} m$. Thus, it is expected to lead up to numerical coefficients of order $\mathcal{O}(1)$ to the same behavior in $\gamma$ as the energy emitted in the scalar case. On the basis of the qualitative arguments given in the beginning of section 4 of [106] and the subsequent numerical computations one obtains ${ }^{16}$ for the emitted energy in the various angular-frequency domains the behavior summarized in table 2 below.

\subsection{The total radiated energy}

According to table 2 the frequency and angular distribution of the dominant component of radiation depends on the dimensionality $d$. Thus, like in the scalar case [106] the study of the emitted energy has to proceed separately for $d \geqslant 2$ and for $d=0,1$. Furthermore, the following analysis is a triple repetition of the scalar case, because of the three polarizations.

\footnotetext{
${ }^{16} \mathrm{Up}$ to a logarithmic overall factor for $d=1$, discussed below.
} 


\begin{tabular}{|c|c|c|c|c|}
\hline$\omega_{D}$ & $\omega_{D} \ll \gamma / b$ & $\omega_{D} \sim \gamma / b$ & $\omega_{D} \sim \gamma^{2} / b$ & $\omega_{D} \gg \gamma^{2} / b$ \\
\hline$\gamma^{-1}$ & $\begin{array}{c}\text { negligible } \\
\text { (phase space) }\end{array}$ & $\begin{array}{l}E \sim \gamma^{3}, \\
\text { from } T \text { and } S\end{array}$ & $\begin{array}{l}E \sim \gamma^{d+2}, \\
\text { from } T+S^{[z]}\end{array}$ & $\begin{array}{r}\text { negligible } \\
\text { radiation }\end{array}$ \\
\hline 1 & $\begin{array}{c}\text { negligible } \\
\text { (phase space) }\end{array}$ & $\begin{array}{l}E \sim \gamma^{d+1} \\
\text { from } S^{\left[z^{\prime}\right]}\end{array}$ & $\begin{array}{l}\text { negligible } \\
\text { radiation }\end{array}$ & $\begin{array}{l}\text { negligible } \\
\text { radiation }\end{array}$ \\
\hline
\end{tabular}

Table 2. The relative contribution in the total emitted energy from different characteristic regions of angle and frequency. All estimates are given in units $\lambda=b=1$.

$\boldsymbol{d} \geqslant \mathbf{2}$. For $d \geqslant 2$ the dominant radiation is beamed with characteristic frequency around $\omega \sim \gamma^{2} / b$. According to table 1 , the emission amplitude is $\mathcal{O}\left(\gamma^{-1}\right)$, dominated by its real part.

For $R \gg b$ the KK-summation is replaced by integration and the relevant formula for the emitted energy is (2.43). One next substitutes the high-frequency amplitudes (3.34), (3.36) and (3.39) corresponding to the three polarizations and neglects their behavior at smaller frequencies. Squaring each amplitude, one recognizes the appearance of products of $K_{d / 2}(z), \hat{K}_{d / 2+1} / z^{2}$ and $\hat{K}_{d / 2+2} / z^{2}$ with themselves, and also the presence of the factor $\omega^{d+2}$ from phase space. Thus, one starts with

$$
\frac{d E}{d \Omega}=\frac{\varkappa_{D}^{2}}{4(2 \pi)^{D-1}} \sum_{\mathcal{P}} \int_{0}^{\infty} \omega^{D-2} d \omega\left|\tau_{L R}(k) \varepsilon_{\mathcal{P}}^{L R}\right|^{2}
$$

and upon integration over the angles except $\vartheta$ one obtains

$$
\frac{d E}{d \vartheta}=\frac{\left(\varkappa_{D}^{3} m m^{\prime}\right)^{2} \gamma^{d+1}}{8(2 \pi)^{2 d+5} b^{3 d+3}} \frac{\sin ^{d+3} \vartheta}{\psi^{d+3}} \sum_{a, b=0}^{2} C_{a b}^{(d)} D_{a b}^{(d)}(\vartheta)
$$

where

$$
C_{a b}^{(d)} \equiv \int \hat{K}_{d / 2+a}(z) \hat{K}_{d / 2+b}(z) z^{d+2\left(\delta_{0 a}+\delta_{0 b}-1\right)} d z
$$

$D_{00}^{(d)}(\vartheta)=2 \frac{(d+1)^{3}}{(d+2)^{3}} \frac{\sin ^{2} \vartheta}{\gamma^{2} \psi^{2}} \quad D_{01}^{(d)}(\vartheta)=(d+2) D_{00}^{(d)}(\vartheta)$

$D_{11}^{(d)}(\vartheta)=(d+2)^{2} D_{00}^{(d)}(\vartheta) \quad D_{02}^{(d)}(\vartheta)=\frac{d+1}{(d+2)^{2}}\left[d-(d+1) \frac{\sin ^{2} \vartheta}{\gamma^{2} \psi^{2}}\right]$

$D_{12}^{(d)}(\vartheta)=(d+2) D_{02}^{(d)}(\vartheta)$

$$
D_{22}^{(d)}(\vartheta)=\frac{d+1}{d+2}\left(2 \frac{\gamma^{2} \psi^{2}}{\sin ^{2} \vartheta}+\frac{3}{4} \frac{\sin ^{2} \vartheta}{\gamma^{2} \psi^{2}}\right)+\frac{2}{d+2}+\gamma^{2} \sin ^{2} \vartheta .
$$

(all of $\mathcal{O}(1)$ for $\vartheta \sim 1 / \gamma$ ), while use was made of (C.5):

$$
\int_{S^{d+1}} d \Omega_{d+1}=\Omega_{d+1}, \quad \int_{S^{d+1}} \cos ^{2} \phi d \Omega_{d+1}=\frac{1}{2} \Omega_{d+1}, \quad \int_{S^{d+1}} \cos ^{4} \phi d \Omega_{d+1}=\frac{3}{8} \Omega_{d+1} .
$$


Upon integration over $\vartheta$ using (C.2) one ends up with

$$
E=\frac{\pi^{d / 2+1}\left(\varkappa_{D}^{3} m m^{\prime}\right)^{2} \gamma^{d+2}}{8(2 \pi)^{2 d+5} \Gamma(d / 2+1) b^{3 d+3}} \sum_{a, b=0}^{2} C_{a b}^{(d)} D_{a b}^{(d)},
$$

where the constants $D_{a b}^{(d)}$ are given by

$D_{00}^{(d)}=2^{d+5} \frac{(d+1)^{3}}{(d+2)^{3}} \frac{\Gamma\left(\frac{d+6}{2}\right) \Gamma\left(\frac{d+4}{2}\right)}{\Gamma(d+5)} \quad D_{02}^{(d)}=-2^{d+3} \frac{(d+1)(2 d+3)}{(d+2)^{2}} \frac{\Gamma\left(\frac{d+4}{2}\right) \Gamma\left(\frac{d+2}{2}\right)}{\Gamma(d+4)}$

$D_{01}^{(d)}=(d+2) D_{00}^{(d)} \quad D_{12}^{(d)}=(d+2) D_{02}^{(d)}$

$D_{11}^{(d)}=(d+2)^{2} D_{00}^{(d)}$

$D_{22}^{(d)}=2^{d-2}(5 d+6)\left(3 d^{2}+15 d+20\right) \frac{\Gamma\left(\frac{d+2}{2}\right) \Gamma\left(\frac{d}{2}\right)}{\Gamma(d+4)}$.

Finally, after the summation in (4.7) one obtains for the total emitted energy in the lab frame

$$
E \simeq C_{d} \frac{\left(\varkappa_{D}^{3} m m^{\prime}\right)^{2}}{b^{3 d+3}} \gamma^{d+2}
$$

with $C_{2}=6.23 \times 10^{-4}, C_{3}=2.61 \times 10^{-4}, C_{4}=1.98 \times 10^{-4}, C_{5}=2.16 \times 10^{-4}, C_{6}=$ $3.10 \times 10^{-4}$.

$\boldsymbol{d}=1$. The emitted energy flux as well as its frequency and angular distributions in $d=0,1$ were computed numerically.

According to tables 1 and 2 in this case most of the radiation is beamed $\vartheta \lesssim 1 / \gamma$, but, as it was described in [106] and immediately follows from the destructive interference, in five dimensions the frequency distribution falls as $1 / \omega$ in the region from $\mathcal{O}(\gamma / b)$ to $\mathcal{O}\left(\gamma^{2} / b\right)$, hence the total emitted energy grows as $\gamma^{3} \ln \gamma$, while the dominant contribution comes from this entire region. From the known expressions for the amplitudes in integral form, we compute the integral numerically for several values of $\gamma$ and conclude:

$$
E \simeq C_{1} \frac{\left(\varkappa_{5}^{3} m m^{\prime}\right)^{2}}{b^{6}} \gamma^{3} \ln \gamma, \quad C_{1}=1.66 \times 10^{-4} .
$$

$\boldsymbol{d}=\mathbf{0}$. Again, according to tables 1 and 2 in this case most of the radiation is beamed within $\vartheta \lesssim 1 / \gamma$ and with frequencies around $\omega \sim \gamma / b$, with the contribution from higher frequencies decaying as $1 / \omega^{2}$. In this domain the local and non-local parts of the amplitude are equally important. Thus, one has to add (3.17) and (3.25) to compute the total radiation amplitude, project onto the two polarizations $\epsilon_{ \pm}$, square each one of those, add them up and multiply by the appropriate phase space factor. After the angular integration one obtains the frequency (or $z^{\prime}$ ) distribution of the emitted energy shown in figure 3a, while the angular distribution of the total emitted energy (which carries the anisotropy) is given in figure $3 \mathrm{~b}$.

Integrating the frequency distribution numerically over $\omega$ from 0 to $+\infty$ one obtains for the total emitted energy

$$
E \simeq C_{0} \frac{\left(\varkappa_{4}^{3} m m^{\prime}\right)^{2}}{b^{3}} \gamma^{3}, \quad C_{0}=5.7 \times 10^{-4} .
$$




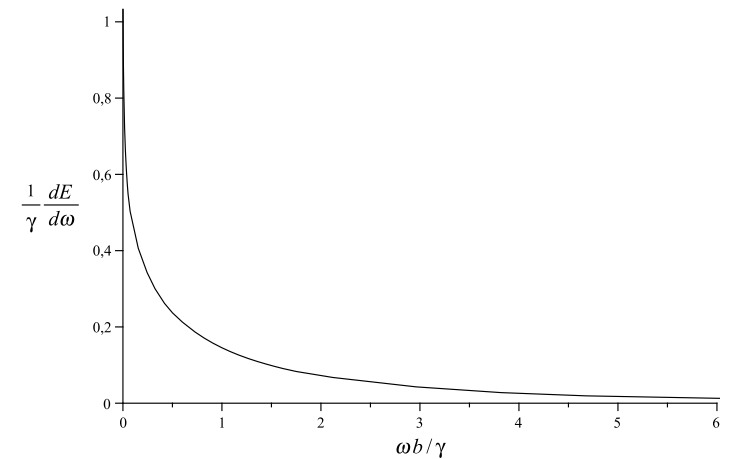

(a)

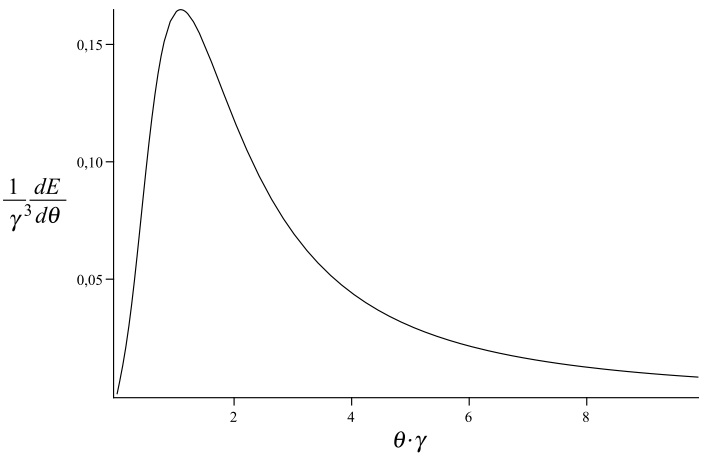

(b)

Figure 3. Frequency (a) and angular (b) distribution of the total emitted energy for $d=0$ and $\gamma=10^{3}$.

\subsection{The low frequency part of the spectrum}

A few comments, related to the low frequency part of the classical radiation spectrum, are in order here.

(a) For $\boldsymbol{d}=\mathbf{0}$ the distribution $d E / d \omega$ of the emitted energy has non-vanishing finite zero-frequency limit.

Indeed, comparing local (3.17) and stress (3.25) sources one concludes that for $\omega \rightarrow$ +0 the dominant part of the amplitude is given by the imaginary part of the local piece $^{17}$ (omit the phase factor $e^{i(k b)}$ ), whose behavior is

$$
\tau_{M N}(k) \simeq-i \frac{\lambda \Gamma}{\gamma v^{3}} \frac{\hat{K}_{1}(z)}{z^{2}} \sigma_{M N} \sim \frac{1}{\omega} .
$$

Upon contraction with the polarizations $\varepsilon_{ \pm}$, substitution of the finite zero-limit $\hat{K}_{1}(0)=1$ of the hatted Macdonald function, and setting $v=1, \Gamma=1$, one obtains

$$
\tau_{ \pm} \simeq \frac{i \lambda}{2} \frac{\gamma \sin \vartheta}{\omega b}\left[\cos \phi\left(\frac{1}{\gamma^{2} \psi^{2}}-1\right) \pm 2 i \frac{\sin \phi}{\psi}\right]
$$

Substitute this into (4.2) and integrate over the azimuthal angle $\phi$, to obtain

$$
\left.\frac{d E}{d \omega}\right|_{\omega=0}=\frac{\varkappa_{4}^{2} \lambda^{2} \gamma^{2}}{16(2 \pi)^{2} b^{2}} \int d \vartheta \sin ^{3} \vartheta\left(1+\frac{4}{\psi^{2}}+\frac{1}{\gamma^{4} \psi^{4}}\right)
$$

plus subleading powers of $\gamma$. Note that in the low frequency limit all angles $(0 \leqslant \vartheta \leqslant$ $\pi)$ contribute to $d E / d \omega$.

\footnotetext{
${ }^{17}$ In the diagrammatic quantum mechanical treatment of soft gravitational radiation there is a corresponding statement, namely that the soft radiation emitted from internal lines is negligible. Only gravitons attached on the external lines contribute in the zero-frequency limit.
} 
Finally, using $V_{0}^{3} \simeq 4 / 3$ (C.3), $V_{4}^{3} \simeq 4 \gamma^{4} / 3$ (C.2), $V_{2}^{3} \simeq 4(\ln 2 \gamma-1)$ (C.4), and substituting the expression for $\lambda$, one ends-up with

$$
\left.\frac{d E}{d \omega}\right|_{\omega=0}=\frac{\left(\varkappa_{4}^{3} m m^{\prime}\right)^{2}}{4(2 \pi)^{4} b^{2}} \gamma^{2}\left(\ln 2 \gamma-\frac{5}{6}\right)
$$

which for $\varkappa_{4}=m=m^{\prime}=b=1$ and $\gamma=10^{3}$ agrees with the value obtained numerically in figure $3 a^{18}$

(b) One can multiply the above result for $d E /\left.d \omega\right|_{\omega=0}$ by the range $\omega_{\max } \sim \gamma / b$ to obtain a rough estimate of the total emitted energy. Its $\gamma$ dependence differs from (4.11) by a logarithmic factor.

However, this simple method to obtain an order of magnitude estimate cannot be used in $d>0$, because according to table 2 the dominant contribution to the emitted energy comes from the high frequency regime, which does not have overlap with the $\omega \rightarrow 0$ domain.

(c) For $\boldsymbol{d}>\mathbf{0}$, one cannot replace at small frequencies the KK-mode summation with integration. One has instead to keep separately the three-dimensional coordinates $\varpi, \theta, \varphi$ and the extra-dimensional discrete momenta $k_{T}$. In this case

$$
z=\frac{(k u) b}{\gamma v}=\frac{b}{v}\left(\sqrt{\varpi^{2}+k_{T}^{2}}-\varpi v \cos \theta\right) \quad \text { and } \quad z^{\prime}=\frac{\left(k u^{\prime}\right) b}{\gamma v}=\frac{b}{\gamma v} \sqrt{\varpi^{2}+k_{T}^{2}},
$$

while $(k b)=-\varpi \sin \theta \cos \varphi$.

The real part inside the square bracket of (3.13) is regular or blows-up logarithmically at $\varpi=0$. Thus, it vanishes when multiplied by the volume measure. Consequently, the dominant contribution to the emitted energy is due to the imaginary part, i.e. (ignoring the irrelevant phase factor)

$$
\tau_{M N}(k) \simeq{ }^{1} T_{M N}(k) \simeq \frac{i m m^{\prime} \varkappa_{D}^{2}}{4 \pi V} \frac{1}{\gamma v^{3}} \sum_{l \in \mathbb{Z}^{d}} \frac{\hat{K}_{1}\left(z_{l}\right)}{z^{2}} \sigma_{M N} .
$$

Taking into account that $\hat{K}_{1}\left(z_{l}\right)$ is regular at all frequencies, the low-frequency behavior is determined by the factor $\sigma_{M N} / z^{2} \sim \omega / z^{2}$. For $k_{T}^{2}>0$ this limit is zero and does not contribute. For $k_{T}=0$ we have $\varpi=\omega$ and thereby $\sigma_{M N} / z^{2} \sim 1 / \omega$ as in four dimensions. Thus, as expected, only the zeroth emission mode $n=0$ contributes in the summation over $n$ of (2.53) in the $\omega \rightarrow 0$ limit.

Now, for $k_{T}=0 z=\omega b \psi(\theta) / v$ with $\psi(\theta) \equiv 1-v \cos \theta$ and

$$
\tau_{M N}(k) \simeq \frac{m m^{\prime} \varkappa_{D}^{2}}{4 \pi \gamma V} \sum_{l \in \mathbb{Z}^{d}} \frac{\hat{K}_{1}\left(\left|p_{T}\right| b\right)}{\omega^{2} b^{2} \psi^{2}(\theta)} \sigma_{M N}, \quad p_{T}^{i}=\frac{l^{i}}{R} .
$$

\footnotetext{
${ }^{18}$ Note that this result differs from the one obtained in [57], where it was claimed that $d E / d \omega$ blows-up logarithmically as $\omega \rightarrow 0$.
} 
For $b \ll R$, which is the case of interest here, the summation over the interaction-KK modes can be restricted up to modes with $\left|p_{T}\right| b \sim 1$. The result, which can also be obtained by the usual substitution of the summation with integration, is

$$
\tau_{M N}(k) \simeq \frac{\lambda}{\gamma} \frac{2^{d / 2} \Gamma(d / 2+1)}{\omega^{2} b^{2} \psi^{2}(\theta)} \sigma_{M N}
$$

and subsequently, using (2.53), one concludes that

$$
\left.\frac{d E}{d \omega}\right|_{\omega=0}=\frac{2^{d-2} \Gamma^{2}(d / 2+1)\left(\varkappa_{D}^{3} m m^{\prime}\right)^{2}}{(2 \pi)^{d+4} V b^{2 d+2}} \gamma^{2}\left[\ln 2 \gamma+\frac{1}{6}\left(\frac{d}{d+2}-5\right)\right] .
$$

The volume $V$ of the internal space survives in the denominator, because, as explained above, only the $n=0$ mode of radiation contributes. Of course, for $d=0$ (4.19) coincides with (4.15).

(d) In $\mathcal{M}_{D>4}$ the frequency spectrum vanishes for $\omega \rightarrow 0$. There are many ways one can convince himself about this. First, it is obtained by taking the limit $V \rightarrow \infty$ of (4.19). Alternatively, observe that in higher dimensional Minkowski the relative contribution on the various radiation modes satisfies $\sum_{n=0} / \sum_{n^{2}>0} \rightarrow 0$. Taking in addition into account that non-zero modes $n$ do not contribute in the limit $\omega \rightarrow 0$, one concludes that $d E /\left.d \omega\right|_{\omega=0}$ vanishes in higher dimensions. Finally, the same conclusion is reached by taking the behavior $\tau \sim 1 / \omega$, square and multiply by the measure $\omega^{d+2}$. Again, for $d>0$ the limit of the integrand for $\omega \rightarrow 0$ is zero.

\subsection{Quantum constraints}

The above results were obtained in the context of classical theory. However, quantum mechanics restricts the region of validity of a classical computation. Thus, to ensure the reliability of the above conclusions, apart from the condition $b \ll R$, which allows one to replace the summations over KK modes with integrations, and which will be assumed in the sequel, one has to justify (a) the use of trajectories for the colliding particles, (b) the expansion around flat space for the gravitational field, and (c) the use of classical field description of the radiation. From the analysis of the elastic ultrarelativistic scattering problem [120] one concludes that the classical computation around flat space-time of the cross-section is identical to the eikonal approximation of the quantum answer, as long as the conditions

$$
\sqrt{s}=2 m \gamma_{\mathrm{cm}} \gg M_{*}, \quad b>b_{\gamma} \equiv r_{S} \gamma_{\mathrm{cm}}^{1 /(d+1)}, \quad b<b_{c} \equiv r_{S}\left(r_{S} / \lambda_{B}\right)^{2 / d}
$$

are satisfied. ${ }^{19}$ For the radiation problem at hand, the weak particle-recoil condition due to the emission of gravitons with characteristic frequency $\omega$ is satisfied if the momenta of the emitted gravitons are much smaller than the momentum transfer of the elastic collision. However, experience from analogous computations of the total energy of synchrotron radiation shows that this condition can be relaxed and replaced, instead, by the weaker

\footnotetext{
${ }^{19}$ For colliding particles with equal masses $\gamma_{\mathrm{cm}}^{2}=(1+\gamma) / 2 \sim \gamma / 2$
} 
$\omega \ll \mathcal{E}_{0}$, where $\mathcal{E}_{0} \simeq m \gamma$ is the total energy of the colliding particles. When the emitted energy $E$ is of order $\mathcal{E}_{0}$, this condition also guarantees a large number of emitted quanta, and justifies further the description of radiation with a classical field. ${ }^{20}$

\subsubsection{The $\omega \sim \gamma^{2} / b$ and $\omega \sim 1 / b$ regimes}

- Radiation with characteristic frequency $\omega \sim \gamma^{2} / b$ does not satisfy the above constraints, and consequently may not be reliably treated classically in the presence of extra dimensions. Indeed, for $\omega \sim \gamma^{2} / b$ the condition $\omega<m \gamma$ leads to $b>\gamma / m>b_{c}$, which contradicts the requirement $b<b_{c}$. Thus, in the sequel we shall ignore the radiation with characteristic frequency $\gamma^{2} / b$, even though according to table 2 it is classically dominant for $d \geqslant 2$.

- On the contrary, as explained in [106] and shown in table 2 the radiation emitted in the soft frequency regime $\omega \lesssim 1 / b$, satisfies all the above conditions, but its energy is negligible by phase space in all dimensions, and will not be discussed further.

\subsubsection{The $\omega \sim \gamma / b$ regime}

According to table 2 the dominant radiation in this regime is beamed for $d=0,1$, almost isotropic for $d \geqslant 3$, while for $d=2$ the two components are of comparable importance.

For $\omega \sim \gamma / b$ the quantum condition $\omega<m \gamma$ reduces to $m b>1$.

Thus, in terms of the rescaled dimensionless parameters

$$
\mathcal{E} \equiv \frac{\sqrt{s}}{M_{*}}, \quad \mu \equiv \frac{m}{M_{*}}, \quad \beta \equiv b M_{*}
$$

a reasonable set of requirements for the validity of the above classical results in this intermediate frequency regime is: ${ }^{21}$

$$
\mathcal{E} \gg 1, \quad \mu \ll 1, \quad \beta>\left(\frac{\mathcal{E}^{2}}{\mu}\right)^{1 /(d+1)}, \quad \beta<\mathcal{E}^{2 / d}, \quad \beta \mu>1,
$$

which are equivalent to

$$
\mathcal{E} \gg 1, \quad \mu \ll 1, \quad \frac{1}{\mu}<\left(\frac{\mathcal{E}^{2}}{\mu}\right)^{1 /(d+1)}<\beta<\mathcal{E}^{2 / d},
$$

and can easily be satisfied in all dimensions. ${ }^{22}$

Thus, we shall next compute the energy emitted in the $\omega \sim \gamma / b$ characteristic frequency regime. $^{23}$

\footnotetext{
${ }^{20}$ In what follows and in order to simplify notation we replace the strong inequalities by simple ones.

${ }^{21}$ The condition $\mu \ll 1$ is necessary to justify the use of point-particle, instead of black-hole description of the colliding particles.

${ }^{22}$ For $d=0$ there is no upper bound on the allowed values of $\beta$.

${ }^{23}$ Incidentally, this is the frequency regime in which one would a priori expect to have dominant radiation: due to the Lorentz squeezing of the field lines along the direction of relative motion of the colliding particles, the characteristic duration of the collision in the lab frame becomes $\tau \sim b / \gamma$. Thus, the characteristic frequency of the emitted radiation should be $\omega \sim 1 / \tau \sim \gamma / b$.
} 


\subsection{Radiation emission with characteristic frequency $\omega \sim \gamma / b$}

As explained above, the estimates in table 2 were obtained under the assumption that the frequency integrations could be restricted to the appropriate regime corresponding to each entry, i.e. from a fraction of the corresponding characteristic frequency to a few times that frequency. The quantum conditions discussed above restrict $\omega$ to $\omega \leqslant \omega_{\max }=m \gamma$, which translates to $z_{\max }^{\prime}=m b>1$.

\subsection{1 $d \geqslant 3$}

Thus, for $d \geqslant 3$, according to tables 1 and 2 the leading contribution to the emitted energy is obtained from (3.44) integrated over phase space with $z^{\prime}$ up to $z_{\max }^{\prime}$. But, the fact that the integrand is exponentially suppressed beyond $z^{\prime} \sim 1$, allows one with negligible error (in the overall coefficient) to extend the $z^{\prime}$ integration to $+\infty$. As far as the lower limit is concerned it is a priori $1 / b$, since (3.44) is valid for $\omega>\mathcal{O}\left(b^{-1}\right)$. However, since the integrand in (4.4) is regular at $z^{\prime}=0$ one can shift the lower limit of integration to 0 , the relative error being of order $1 / \gamma^{2}$. This reproduces the power of $\gamma$ estimated in table 2 . The purpose of the rest of this subsection is essentially to obtain the overall coefficient.

Projecting (3.44) onto $\varepsilon_{ \pm}$and $\varepsilon_{\mathrm{III}}$ one obtains

$$
\begin{gathered}
\tau_{ \pm} \simeq \frac{\lambda}{2{z^{\prime}}^{2} \gamma \psi}\left[\left(\cos ^{2} \phi \cos ^{2} \vartheta-\sin ^{2} \phi \pm i \sin 2 \phi \cos \vartheta\right) \hat{K}_{d / 2+2}\left(z^{\prime}\right)-\right. \\
\left.-\sin ^{2} \vartheta\left(\hat{K}_{d / 2+1}\left(z^{\prime}\right)+\frac{1}{d+2} z^{\prime 2} \hat{K}_{d / 2}\left(z^{\prime}\right)\right)\right] \\
\tau_{\text {III }} \simeq-\sqrt{\frac{d}{2(d+2)}} \frac{\lambda}{z^{\prime 2} \gamma \psi}\left[\left(1-\cos ^{2} \phi \sin ^{2} \vartheta\right) \hat{K}_{d / 2+2}\left(z^{\prime}\right)-\right. \\
\left.-\sin ^{2} \vartheta\left(\hat{K}_{d / 2+1}\left(z^{\prime}\right)+\frac{1}{d+2} z^{\prime 2} \hat{K}_{d / 2}\left(z^{\prime}\right)\right)\right] .
\end{gathered}
$$

One then according to (2.43) has to square these, multiply with the phase space factor and integrate over angles and frequencies.

The result of the integration over frequencies takes the form

$$
\frac{d E}{d \Omega_{d+2}}=\frac{\left(\varkappa_{D}^{3} m m^{\prime}\right)^{2} \gamma^{d+1}}{16(2 \pi)^{2 d+5} b^{3 d+3} \psi^{2}} \sum_{a, b=0}^{2} C_{a b}^{(d)} \bar{D}_{a b}^{(d)}(\vartheta, \phi),
$$

where $C_{a b}^{(d)}$ are given by (4.4) and

$$
\begin{array}{ll}
\bar{D}_{00}^{(d)}(\vartheta, \phi)=\frac{d+1}{(d+2)^{3}} \sin ^{4} \vartheta & \bar{D}_{01}^{(d)}(\vartheta, \phi)=(d+2) \bar{D}_{00}^{(d)}(\vartheta, \phi) \\
\bar{D}_{11}^{(d)}(\vartheta, \phi)=(d+2)^{2} \bar{D}_{00}^{(d)}(\vartheta, \phi) & \bar{D}_{02}^{(d)}(\vartheta, \phi)=-\frac{\sin ^{2} \vartheta}{d+2}\left[\cos ^{2} \phi+\frac{2}{d+2}\left(\cos ^{2} \phi \sin ^{2} \vartheta-1\right)\right] \\
\bar{D}_{12}^{(d)}(\vartheta, \phi)=-(d+2) \bar{D}_{02}^{(d)}(\vartheta, \phi) & \bar{D}_{22}^{(d)}(\vartheta, \phi)=\frac{d+1}{d+2}\left(1-\cos ^{2} \phi \sin ^{2} \vartheta\right)^{2}
\end{array}
$$

The values of $C_{a b}^{(d)}$ are to be computed with help of (C.6). 
The integration of (4.26), (4.25) over the angles except $\vartheta$ (i.e. over the sphere $S^{d+1}$ ) is easily performed using formulae (4.6), while the final integration over $\vartheta$ is done with the help of (C.3). ${ }^{24}$ Making use also of (4.4), one finally obtains for the emitted energy

$$
E=\bar{C}_{d} \frac{\left(\varkappa_{D}^{3} m m^{\prime}\right)^{2}}{b^{3 d+3}} \gamma^{d+1},
$$

with $\bar{C}_{3}=1.08 \times 10^{-4}, \bar{C}_{4}=4.77 \times 10^{-5}, \bar{C}_{5}=4.03 \times 10^{-5}$ and $\bar{C}_{6}=4.98 \times 10^{-5}$.

\subsection{2 $d=0$}

As explained above, the $z^{\prime}$ integration extends a priori up to $z_{\max }^{\prime} \sim m b>1$. It was checked numerically that (a) the estimate of the cubic power of $\gamma$ given in table 2 is correct, and (b) that the coefficient is insensitive to the upper limit of integration, varying by a factor of two as one changes the upper limit of the $z^{\prime}$ integration between 1 and $\infty$.

Thus, (4.11) is a reliable estimate.

\subsection{3 $d=1,2$}

In this case the estimation of the contribution to the emitted energy from the $\omega \sim \gamma / b$ part of the spectrum was done numerically. Specifically, one started with (3.17) and (3.25) and integrated numerically over $\vartheta$ from 0 to $\pi$ and over $z^{\prime}$ from 0 to something of order 1 . For a reliable estimate it is enough to integrate up to $z^{\prime}=1$.

The result for $d=2$ is

$$
E \simeq 0.04 \frac{\left(\varkappa_{6}^{3} m m^{\prime}\right)^{2}}{b^{9}} \gamma^{3} \ln \gamma
$$

and for $d=1$

$$
E \simeq 0.9 \times 10^{-4} \frac{\left(\varkappa_{5}^{3} m m^{\prime}\right)^{2}}{b^{6}} \gamma^{3}
$$

\subsection{Extreme radiation efficiency for $d \geqslant 3$ and $\omega \sim \gamma / b$}

Consider specifically the case $m=m^{\prime}$, divide the emitted energy by the total energy $\mathcal{E}_{0}=m+m \gamma$ of the colliding particles and use the definition of $r_{S}$ given in the Introduction, to write the radiation efficiency $\epsilon \equiv E / \mathcal{E}_{0}$ in the form ${ }^{25}$

$$
\epsilon \sim\left(\frac{r_{S}}{b}\right)^{3(d+1)} \gamma_{\mathrm{cm}} \sim \frac{\mathcal{E}^{4}}{\mu \beta^{3(d+1)}}, \quad d=0,1
$$

and

$$
\epsilon \sim\left(\frac{r_{S}}{b}\right)^{3(d+1)} \gamma_{\mathrm{cm}}^{2 d-3} \sim \frac{\mathcal{E}^{2 d}}{\beta^{3(d+1)} \mu^{2 d-3}}, \quad d \geqslant 2
$$

\footnotetext{
${ }^{24}$ At small angles expressions (4.24) are not valid. But as before, if we substitute integral $\int_{\mathcal{O}(1 / \gamma)}^{\pi} d \theta$ by $\int_{0}^{\pi} d \theta$, the relative error is $\mathcal{O}(1 / \gamma)$ since the integrand doesn't blow up at $\vartheta=0+\mathcal{O}(1 / \gamma)$. Thus we may apply (C.3).

${ }^{25}$ Since it is not our intention to engage in numerology, we ignore in these formulae inessential d-dependent coefficients, even though they are roughly of $\mathcal{O}\left(10^{d}\right)$, or the factor $\ln \gamma$, which is present for $d=2$.
} 
It should be pointed out that these expressions differ from the generic formula $\epsilon \sim$ $\left(r_{S} / b\right)^{3(d+1)} \gamma_{\mathrm{cm}}^{2 d+1}$ given erroneously in [107]. The difference is due to an error in [107] related to the extent of destructive interference. Nevertheless, the qualitative conclusions presented in [107] are still valid. Incidentally, the powers of $\gamma$ in the emitted energy differ from the scalar radiation studied in [106] only by the substitution $f \rightarrow \varkappa_{D} m$.

In the parameter range defined in (4.23) the radiation efficiency is always much smaller than one for $d=0,1,2$. Indeed, consider the case $d=0$. The maximum $\epsilon$ is obtained for the minimum allowed value $\beta \sim\left(\mathcal{E}^{2} / \mu\right)^{1 /(d+1)}$ of the impact parameter and is equal to

$$
\epsilon_{\max }(d=0) \sim\left(\frac{\mu}{\mathcal{E}}\right)^{2} \ll 1 .
$$

Thus, in $d=0$ a small fraction of the available energy is emitted in gravitational radiation and vanishes in the massless limit. However, one can convince her/himself that for $d \geqslant 3$ the efficiency can take values arbitrarily close to one or even become much greater than one for a wide range of parameters obeying the classicality conditions (4.23) stated above. For instance, in $d=4$ the minimum value of $\epsilon$ is obtained for $\beta \sim \mathcal{E}^{2 / d}$ and is

$$
\epsilon_{\min }(d=4) \sim \frac{\sqrt{\mathcal{E}}}{\mu^{5}} \gg 1 .
$$

Ditto for dimensions $d>4$. This is a priori a puzzling result. It leads for $d \geqslant 3$ to unphysical, i.e. greater than one, values of the radiation efficiency, and actually blows-up in the massless limit $(\mu \rightarrow 0)$. In relation to this we would like to offer the following comments: (a) The massless limit is a very singular limit in our approach. The characteristic frequencies and angles all become singular in that limit. In particular, since in that limit $b_{c} / b_{\gamma} \sim\left(\mathcal{E}^{2 / d} \mu\right)^{1 /(d+1)} \rightarrow 0$, it is impossible to satisfy (4.23), even if one ignores the "quantum" constraint $\beta \mu>1$. Thus, the massless limit lies outside the domain of validity of our approximation. (b) Weinberg has shown [119] that in $d=0$ there is no infrared divergence in the soft graviton emission rate from massless colliding particles. This is in agreement with our results not only for the soft component in $d=0$, but also for soft as well as high frequency emission in $d=0,1,2$. (c) The explicit computation presented here is rather involved, but, as explained, the powers of $\gamma$ in the emitted energy are determined on general grounds, based on properties of Macdonald functions, number of KK interaction and emission modes and dimensionality of phase space. (d) One may suspect that some classicality condition is missed, which might render the above formulae non-applicable. But the constraints used in (4.23) are quite straightforward and based on well-known physics. So, (e) it may well be the case, that the above results are reliable and the interpretation of their puzzling features is that in ultra-planckian particle collisions there is a lot of energy emitted in gravitational radiation. This will lead to strong radiation damping, which should be taken into account in the study of such ultra-planckian scattering processes.

\section{Conclusions}

A detailed study was presented of classical gravitational radiation emitted in ultra-relativistic collisions of massive point-particles interacting gravitationally. The space-time was 
assumed to have an arbitrary number of toroidal or non-compact extra dimensions and the post-linear approximation scheme of General Relativity was employed for the computation. The angular and frequency distributions of radiation, as well as the total emitted energy were studied in detail to leading ultra-relativistic order.

Three characteristic frequency regimes $\left(1 / b, \gamma / b\right.$ and $\left.\gamma^{2} / b\right)$ of the emitted radiation were identified and the characteristics of the dominant contribution was determined in various dimensions. In particular, in any number of dimensions the $1 / b$ component of radiation is mainly due to the scattered particles, with negligible contribution coming from the cubic graviton interaction term. ${ }^{26}$ However, in contrast to the four-dimensional case, in any number of extra dimensions $d>0$ the frequency distribution of the emitted radiation vanishes as $\omega \rightarrow 0$ and the total emitted energy at low frequencies is negligible. On the contrary, in the $\gamma / b$ and $\gamma^{2} / b$ parts of the spectrum the cubic graviton interaction and the scattered particles are equally important sources of radiation. In fact it was shown that in any dimension they lead to partial cancelation (destructive interference) of the total beamed radiation amplitude in the high frequency domain, as a result of which the emitted energy in the $\gamma^{2} / b$ frequency regime is reduced by two powers of the Lorentz factor $\gamma$ in the Lab frame.

The relevance of the classical analysis to the full quantum radiation problem was also discussed. The classicality conditions, necessary for the classical treatment to be a good approximation to the full quantum problem were derived and the radiation efficiency $\epsilon$, i.e. the fraction of the initial energy which is emitted in gravitational radiation, was computed for parameter values inside the region of validity of our classical computation. Although for $d=0,1,2, \epsilon$ is smaller than one in the whole allowed range of parameters, it can be arbitrarily close to one or even exceed unity in $d \geqslant 3$. One possible interpretation of this "unphysical" result is that indeed in such ultra-planckian collisions there is a lot of gravitational radiation, which will lead to strong damping, which should be included in a reliable treatment of the scattering process.

However, the implementation of this interpretation, the proper treatment of the massless limit, the extension of the region of validity to impact parameters outside the range (4.23) and the comparison with quantum results based, for example, on string theory, are currently under investigation and, hopefully, will be the subject of a future publication.

\section{Acknowledgments}

We are grateful to Dr. Georgios Kofinas for useful discussions and to Mr. Yiannis Constantinou for help with the numerical work. This work was supported in part by EU grants PERG07-GA-2010-268246, the EU program "Thales" ESF/NSRF 2007-2013 and grant 11-02-01371-a of RFBR. It has also been co-financed by the European Union (European Social Fund, ESF) and Greek national funds through the Operational Program "Education and Lifelong Learning" of the National Strategic Reference Framework (NSRF) under "Funding of proposals that have received a positive evaluation in the 3rd and 4th

\footnotetext{
${ }^{26}$ This is a well known fact, verified easily also in the context of Feynman diagram infrared graviton summation.
} 
Call of ERC Grant Schemes". DG and PS are grateful to the Department of Physics of the University of Crete for its hospitality in various stages of this work. PS is also grateful to the DAAD service for co-funding and to the LMU, Munich, for its hospitality during the final stage of the work.

\section{A Definitions and variations}

\section{A.1 Perturbation theory variation over gravitational constant in flat back- ground}

We use the notation of Weinberg [112].

$$
\begin{aligned}
g_{M N}^{(1)} & =h_{M N} & \Gamma_{M N}^{(1) R} & =\left(h_{M, N}^{R}+h_{N, M}^{R}-h_{M N}^{, R}\right) / 2 \\
\Gamma_{M N}^{(1) N} & =h_{, M} / 2 & \Gamma_{N R}^{(1) M} \eta^{N R} & =0 .
\end{aligned}
$$

The definition of Riemann and Ricci tensors:

$$
R_{N L R}^{M}=\Gamma_{N R, L}^{M}-\Gamma_{N L, R}^{M}+\Gamma_{S L}^{M} \Gamma_{N R}^{S}-\Gamma_{S R}^{M} \Gamma_{N L}^{S}, \quad R_{M N} \equiv R_{M L N}^{L} .
$$

Thus

$$
\begin{aligned}
R_{M N}^{(1)} & =\frac{1}{2}\left(h_{N ; M R}^{R}+h_{M, N R}^{R}-\square h_{M N}-h_{, M N}\right)=-\frac{1}{2} \square h_{M N} \\
R^{(1)} & =-\square h+h_{M N}{ }^{, M N}-R^{M N} h_{M N}=-\frac{1}{2} \square h \\
G_{M N}^{(1)} & =\frac{1}{2}\left(-\square \psi_{M N}-\eta_{M N} \xi_{L}^{{ }^{L}}+\xi_{M, N}+\xi_{N, M}\right)=-\frac{1}{2} \square \psi_{M N} \quad \xi_{M}=\partial^{N} \psi_{M N}=0 .
\end{aligned}
$$

The second-order variations:

$$
\begin{aligned}
\Gamma_{L R}^{(2) M}= & -\frac{1}{2} h^{M N}\left(h_{L N, R}+h_{R N, L}-h_{L R, N}\right) \\
\Gamma_{M L}^{(2) L}= & -\frac{1}{2} h^{L R} h_{L R, M} \quad \Gamma_{N R}^{(2) M} \eta^{N R}=0 \\
R_{M N}^{(2)}= & -\frac{1}{2}\left(h_{M}^{A, B}\left(h_{N B, A}-h_{N A, B}\right)-\frac{1}{2} h_{, M}^{A B} h_{A B, N}+\right. \\
& \left.\quad+h^{A B}\left(h_{M A, N B}+h_{N A, M B}-h_{A B, M N}-h_{M N, A B}\right)\right) \\
R^{(2)}= & -h^{L R} R_{L R}^{(1)}+\eta^{L R} R_{L R}^{(2)}=\frac{1}{2}\left(2 h^{A B} \square h_{A B}-h_{A B, L} h^{A L, B}+\frac{3}{2} h_{A B, L} h^{A B, L}\right) \\
G_{M N}^{(2)}= & R_{M N}^{(2)}-\frac{1}{2} \eta_{M N} R^{(2)}-\frac{1}{2} h_{M N} R^{(1)} .
\end{aligned}
$$

As a result:

$$
\begin{gathered}
G_{M N}^{(2)}=-\frac{1}{2}\left[h^{A B}\left(h_{M A, N B}+h_{N A, M B}-h_{A B, M N}-h_{M N, A B}\right)+h_{M}^{A, B}\left(h_{N B, A}-h_{N A, B}\right)-\right. \\
\left.-\frac{1}{2} h_{, M}^{A B} h_{A B, N}-\frac{1}{2} h_{M N} \square h+\frac{1}{2} \eta_{M N}\left(2 h^{A B} \square h_{A B}-h_{A B, L} h^{A L, B}+\frac{3}{2} h_{A B, L} h^{A B, L}\right)\right] .
\end{gathered}
$$




\section{A.2 Useful kinematical formulae}

The angles in the formulae below are defined in figure 2 .

$$
\begin{aligned}
u^{\mu} & \equiv \gamma(1,0,0, v) \quad u^{\prime \mu} \equiv(1,0,0,0) \quad \varpi \equiv|\mathbf{k}| \quad \cos \alpha=\frac{\varpi}{\omega} \\
\psi & \equiv 1-v \cos \theta \cos \alpha=1-v \cos \vartheta \quad k_{T}^{i}=\frac{n^{i}}{R} \quad p_{T}^{i}=\frac{l^{i}}{R} \\
z^{\prime} & =\frac{\left(k u^{\prime}\right) b}{\gamma v}=\frac{\omega b}{\gamma v} \quad z=\frac{(k u) b}{\gamma v}=\frac{\omega b}{v} \psi=z^{\prime} \gamma \psi \quad z_{l}^{2} \equiv z^{2}+b^{2} p_{T}^{2} \\
\xi^{2} & \equiv 2 \gamma z z^{\prime}-z^{2}-z^{\prime 2}=\varpi^{2} b^{2} \sin ^{2} \theta+b^{2} k_{T}^{2}=(\omega b \sin \vartheta)^{2}=\left(\gamma v z^{\prime} \sin \vartheta\right)^{2} \\
-(k b) & =\xi \cos \phi=\gamma z^{\prime} v \sin \vartheta \cos \phi=\gamma z^{\prime} v \cos \alpha \sin \theta \cos \varphi=\varpi b \sin \theta \cos \varphi \\
\beta & \equiv \gamma z z^{\prime}-z^{2}=\frac{\varpi^{2} b^{2} \cos \vartheta(1-v \cos \vartheta)}{v \cos ^{2} \alpha}=\gamma^{2} z^{\prime 2} \psi(1-\psi)
\end{aligned}
$$

Next we compute non-vanishing products of polarization vectors: using (2.16), (2.51), (2.52) and these definitions one finds the following scalar products

$$
(k u)=\gamma\left(\sqrt{\varpi^{2}+k_{T}^{2}}-\varpi v \cos \theta\right), \quad\left(k u^{\prime}\right)=\sqrt{\varpi^{2}+k_{T}^{2}}, \quad\left(u u^{\prime}\right)=\gamma .
$$

To calculate the scalar product $\left(b e_{2}\right)$ one observes that $\epsilon^{x z 0 \tau 3 \ldots D-2} b_{x} u_{z} u_{0}^{\prime} k_{\tau} e_{3} \ldots e_{D-2}=$ $-b \gamma v k_{\perp}$, where $k_{\perp}$ is the length of the projection of $k^{M}$ onto the subspace orthogonal to $t, x, z$, since $k$ is transverse to all $e_{\alpha}$ 's and the form $\epsilon^{\tau 3 \ldots D-2} k_{\tau} e_{3} \ldots e_{D-2}$, remaining after factoring out the $t, x, z$ subspace, represents the volume of the rectangle. Thus

$$
k_{\perp}=\sqrt{\varpi^{2} \sin ^{2} \theta \sin ^{2} \varphi+k_{T}^{2}}=\omega \sin \phi,
$$

and one finds

$$
\left(b e_{2}\right)=-b\left(\frac{\varpi^{2} \sin ^{2} \theta \sin ^{2} \varphi+k_{T}^{2}}{\varpi^{2} \sin ^{2} \theta+k_{T}^{2}}\right)^{1 / 2}=-b\left(\frac{\cos ^{2} \alpha \sin ^{2} \theta \sin ^{2} \varphi+\sin ^{2} \alpha}{\cos ^{2} \alpha \sin ^{2} \theta+\sin ^{2} \alpha}\right)^{1 / 2}=-b \sin \phi .
$$

Evaluation of the scalar product $\left(e_{1} b\right)$ is straightforward and leads to

$$
\left(b e_{1}\right)=-\frac{b}{2} \frac{\varpi^{2} \sin 2 \theta \cos \varphi}{\sqrt{\left(\varpi^{2} \sin ^{2} \theta+k_{T}^{2}\right)\left(\varpi^{2}+k_{T}^{2}\right)}}=-\frac{b}{v} \cos \phi\left(1-\frac{(k u)}{\gamma\left(k u^{\prime}\right)}\right)=\frac{(k b)}{\left(k u^{\prime}\right)} \cot \vartheta .
$$

Finally, the remaining non-vanishing scalar product is

$$
\left(e_{1} u\right)=\gamma v\left(\frac{\varpi^{2} \sin ^{2} \theta+k_{T}^{2}}{\varpi^{2}+k_{T}^{2}}\right)^{1 / 2}=\gamma v \sqrt{\cos ^{2} \alpha \sin ^{2} \theta+\sin ^{2} \alpha}=\gamma v \sin \vartheta .
$$




\section{B Momentum integrals}

\section{B.1 Basic scalar integral}

The local integrals for massless and massive modes are calculated in [105]. The main integral for stress (also for massive modes) is calculated in [106].

$$
J(k) \equiv \frac{1}{V} \sum_{l} J^{n l}(k), \quad J^{n l}(k)=\int d^{4} p \frac{\delta\left(p u^{\prime}\right) \delta(k u-p u) e^{-i(p b)}}{\left(p^{2}-p_{T}^{2}\right)\left[(k-p)^{2}-\left(k_{T}^{i}-p_{T}^{i}\right)^{2}\right]} .
$$

$\omega \equiv k^{0}=\sqrt{\mathbf{k}^{2}+k_{T}^{2}}$ and $\mathbf{k}$ is a 3 -dimensional vector lying on the 3 -brane, while $k_{T}^{i}=n^{i} / R$ and $p_{T}^{i}=l^{i} / R$ with integers $\left\{n^{i}\right\},\left\{l^{i}\right\}$, are $d$-dimensional discrete vectors, corresponding to the emission and interaction modes, respectively.

Using Feynman parametrization $J^{n l}$ takes the form:

$$
J^{n l}=\int_{0}^{1} d x e^{-i(k b) x} \int d^{4} p \frac{\delta\left[\left(p u^{\prime}\right)+\left(k u^{\prime}\right) x\right] \delta[(p u)-(1-x)(k u)] \mathrm{e}^{-i(p b)}}{\left[p^{2}-\left(k_{T} x-p_{T}\right)^{2}\right]^{2}} .
$$

Integrating over $p^{0}$ and splitting $\mathbf{p}$ into the longitudinal $p_{\|}$(with respect to $\mathbf{u}$ ) and transversal $\mathbf{p}_{\perp}$ parts, integrate over $p_{\|}$. The corresponding covariant splitting of $b^{\mu}$ on temporal, longitudinal and transversal parts reads

$$
b^{\mu}=\left(b u^{\prime}\right) u^{\prime \mu}-\frac{\gamma\left(b u^{\prime}\right)-(b u)}{\gamma^{2} v^{2}}\left(\gamma u^{\prime \mu}-u^{\mu}\right)+b_{\perp}^{\mu}
$$

and denote scalar impact parameter $b$ as

$$
b \equiv \sqrt{-b_{\perp}^{2}}=\left(-b^{2}-\frac{\left[(b u) u^{\prime}-\left(b u^{\prime}\right) u\right]^{2}}{\gamma^{2} v^{2}}\right)^{1 / 2} .
$$

Then, introducing in $\mathbf{p}_{\perp}$ the spherical coordinates $d^{2} \mathbf{p}_{\perp}=\left|\mathbf{p}_{\perp}\right| d \Omega_{1} d\left|\mathbf{p}_{\perp}\right|$ and integrating first over the angles and then over $\left|\mathbf{p}_{\perp}\right|$, one obtains

$$
J^{n l}=\frac{\pi b^{2}}{\gamma v} \int_{0}^{1} d x e^{-i(N b)} \hat{K}_{-1}\left(\zeta_{n l}\right),
$$

with

$$
\begin{aligned}
\zeta_{n l}^{2}(x) & =z^{\prime 2} x^{2}+2 \gamma z z^{\prime} x(1-x)+z^{2}(1-x)^{2}+b^{2}\left(k_{T} x-p_{T}\right)^{2} \\
N_{\mu} & =\frac{1}{\gamma^{2} v^{2}}\left[x\left(k u^{\prime}\right) u_{\mu}^{\prime}-(1-x)(k u) u_{\mu}\right]-\frac{1}{\gamma v^{2}}\left[x\left(k u^{\prime}\right) u_{\mu}-(1-x)(k u) u_{\mu}^{\prime}\right]+x k_{\mu} .
\end{aligned}
$$

Sum up over $l^{\prime}$ s with rule (3.16) to get

$$
J^{n}(k)=\Lambda_{d} \int_{0}^{1} d x e^{-i(N b)} \hat{K}_{d / 2-1}\left(\zeta_{n}\right) ; \quad \Lambda_{d} \equiv \frac{\pi b^{2-d}}{(2 \pi)^{d / 2} \gamma v} .
$$


with

$$
\zeta_{n}^{2}(x)=z^{\prime 2} x^{2}+2 \gamma z z^{\prime} x(1-x)+z^{2}(1-x)^{2} ; \quad \zeta_{n}(0)=z, \quad \zeta_{n}(1)=z^{\prime} .
$$

Finally, fix $\left(b u^{\prime}\right)=(b u)=0$ to get

$$
J^{n}(k)=\Lambda_{d} \int_{0}^{1} d x e^{-i(k b) x} \hat{K}_{d / 2-1}\left(\zeta_{n}\right) .
$$

Thus

$$
J^{n}(k)=J^{[z]}+J^{\left[z^{\prime}\right]} .
$$

In the frequency-angular region $\varrho \sim \omega b \vartheta \gg 1 J^{[z]}$ and $J^{\left[z^{\prime}\right]}$ are given by the series over small $1 / \varrho^{2}$ :

$$
\begin{aligned}
& J^{[z]}=\frac{\Lambda_{d}}{a^{2} \xi^{2}}\left(\beta \hat{K}_{d / 2}(z)-i(k b) \hat{K}_{d / 2+1}(z)-\frac{(d+1) \beta}{a^{2}} \hat{K}_{d / 2+1}(z)+\frac{\beta \sin ^{2} \phi}{a^{2}} \hat{K}_{d / 2+2}(z)\right)+R_{z} \\
& J^{\left[z^{\prime}\right]}=\Lambda_{d} \mathrm{e}^{-i(k b)}\left(\frac{\xi^{2}-\beta}{\xi^{2} a^{2}} \hat{K}_{d / 2}\left(z^{\prime}\right)-i \frac{\cos \phi}{a^{2} \xi} \hat{K}_{d / 2+1}\left(z^{\prime}\right)\right)+R_{z^{\prime}},
\end{aligned}
$$

where

$$
a \equiv \sqrt{\frac{\beta^{2}}{\xi^{2}}+z^{2}}=\frac{z}{\sin \vartheta} .
$$

Thus $J^{n}(k)$ can be split in two parts with drastically different spectral-angular behavior each.

\section{B.2 Vectorial and tensorial integrals}

Vectorial integral is defined by

$$
J_{M}^{n}(k) \equiv \frac{1}{V} \sum_{l} J_{M}^{n l}(k), \quad J_{\mu}^{n l}(k)=\int d^{4} p \frac{\delta\left(p u^{\prime}\right) \delta(k u-p u) e^{-i(p b)}}{\left(p^{2}-p_{T}^{2}\right)\left[(k-p)^{2}-\left(k_{T}^{i}-p_{T}^{i}\right)^{2}\right]} p_{M} .
$$

Thus

$$
J_{M}^{n}(k)=i \frac{\partial J}{\partial b^{M}} .
$$

Substituting $J^{n}(k)$ in the form (B.6) and differentiating, one gets taking (B.3) into account

$$
J_{M}^{n}=\Lambda_{d} \int_{0}^{1} d x e^{-i(N b)}\left[N_{M} \hat{K}_{d / 2-1}\left(\zeta_{n}\right)+i \frac{\hat{b}_{M}}{b^{2}} \hat{K}_{d / 2}\left(\zeta_{n}\right)\right],
$$

with

$$
\hat{b}_{M} \equiv b_{M}-\frac{(b u) u_{M}+\left(b u^{\prime}\right) u^{\prime}{ }_{M}-\gamma\left[(b u) u^{\prime}{ }_{M}+\left(b u^{\prime}\right) u_{M}\right]}{\gamma^{2} v^{2}}, \quad N_{M}=\left(N_{\mu} ; x k_{T}{ }^{i}\right),
$$


where it is convenient to use the following properties of hatted Macdonalds:

$$
\hat{K}_{ \pm n}^{\prime}(x)=-x \hat{K}_{ \pm n-1}(x) .
$$

In the Lab frame one has $\hat{b}_{M}=b_{M}$ and

$$
J_{M}^{n}=\Lambda_{d} \int_{0}^{1} d x e^{-i(k b) x}\left[N_{M} \hat{K}_{d / 2-1}\left(\zeta_{n}\right)+i \frac{b_{M}}{b^{2}} \hat{K}_{d / 2}\left(\zeta_{n}\right)\right] .
$$

Some useful products

$$
\begin{aligned}
& N \cdot u=(k u) \quad N \cdot u^{\prime}=J^{n} \cdot u^{\prime}=0 \quad N \cdot k=\frac{1}{b^{2}}\left[x\left(z^{2}+z^{\prime 2}-2 z z^{\prime} \gamma\right)+\left(\gamma z z^{\prime}-z^{2}\right)\right] \\
& J^{n} \cdot u=(k u) J^{n} \quad N \cdot b=x(k b) \quad N^{2}=\frac{1}{b^{2}}\left[x^{2}\left(z^{2}+z^{\prime 2}-2 z z^{\prime} \gamma\right)-z^{2}\right] .
\end{aligned}
$$

Tensorial integral is defined

$$
J_{M N}(k) \equiv \frac{1}{V} \sum_{l} J_{M N}^{n l}(k), \quad J_{M N}^{n l}(k)=\int d^{4} p \frac{\delta\left(p u^{\prime}\right) \delta(k u-p u) e^{-i(p b)}}{\left(p^{2}-p_{T}^{2}\right)\left[(k-p)^{2}-\left(k_{T}^{i}-p_{T}^{i}\right)^{2}\right]} p_{M} p_{N} .
$$

Thus

$$
J_{M N}^{n}(k)=i \frac{\partial J_{M}^{n}}{\partial b^{N}} .
$$

Substituting $J_{M}^{n}$ in the form (B.13) and differentiating, we have

$$
\begin{aligned}
J_{M N}^{n}=\frac{\Lambda_{d}}{b^{2}} \int_{0}^{1} d x e^{-i(k b) x} & {\left[b^{2} N_{M} N_{N} \hat{K}_{d / 2-1}\left(\zeta_{n}\right)+2 i N_{(M} b_{N)} \hat{K}_{d / 2}\left(\zeta_{n}\right)-\frac{b_{M} b_{N}}{b^{2}} \hat{K}_{d / 2+1}\left(\zeta_{n}\right)-\right.} \\
& \left.-\left(\eta_{M N}+\frac{1}{v^{2} \gamma^{2}}\left[u_{M} u_{N}+u_{M}^{\prime} u_{N}^{\prime}-2 \gamma u_{(M} u_{N)}^{\prime}\right]\right) \hat{K}_{d / 2}\left(\zeta_{n}\right)\right] .
\end{aligned}
$$

Some products:

$$
J_{M N}^{n} u^{N}=(k u) J_{M}^{n} \quad J_{M N}^{n} u^{\prime N}=0 \quad J_{M N} u^{M} u^{N}=(k u)^{2} J^{n} .
$$

Here we see that integrals $J_{M}^{n}, J_{M N}^{n}$ represent the superposition of some scalar integrals of the type

$$
J_{(\sigma, \tau)} \equiv \Lambda_{d} \int_{0}^{1} x^{\sigma} e^{-i(k b) x} \hat{K}_{d / 2+\tau}\left(\zeta_{n}\right) d x
$$

(with $\sigma=0,1,2$ ): for instance

$$
\begin{gathered}
J_{M}^{n}=\frac{1}{v b}\left[\left(\frac{1}{\gamma}\left[z^{\prime} u_{M}^{\prime}+z u_{M}\right]-\left[z^{\prime} u_{M}+z u_{M}^{\prime}\right]+v b k_{M}\right) J_{(1,-1)}+\right. \\
\left.+z\left(u_{M}^{\prime}-\frac{1}{\gamma} u_{M}\right) J_{(0,-1)}+i \frac{v b_{M}}{b} J_{(0,0)}\right]
\end{gathered}
$$

Two integrals $J_{(0,-1)}$ and $J_{(0,0)}$ (i.e. with $\sigma=0$ ) are of the type of the basic scalar integral and thereby known, while the derived one $J_{(1,-1)}$ is new. The computation of integrals with $\sigma=1,2$ represents the goal of next subsection. 


\section{B.3 Derived integrals for stresses}

Now consider (B.19) with $\sigma=1, \tau=-1$ :

$$
J_{(1,-1)}=\Lambda_{d} \int_{0}^{1} x e^{-i(k b) x} \hat{K}_{d / 2-1}\left(\zeta_{n}\right) d x,
$$

If we'd know $J(k)$ exactly (B.21) can be calculated by differentiation:

$$
J_{(1,-1)}=-\frac{i}{\xi} \frac{\partial J_{(0,-1)}}{\partial \cos \phi},
$$

Our strategy consists in the reduction of $J_{(\sigma, \tau)}$ into the superposition of $J_{\left(0, \tau^{\prime}\right)}$ (with some $\tau^{\prime}$ s), for which we already have calculated expressions (B.10), (B.11). Note that for higher $\tau$ we keep $\Lambda_{d}$ and shift index of Macdonalds in (B.10), (B.11): thereby (B.10), (B.11) may be generalized into

$$
\begin{aligned}
& J_{(0, \tau-1)}^{[z]}=\frac{\Lambda_{d}}{a^{2} \xi^{2}}\left(\beta \hat{K}_{d / 2+\tau}(z)-i(k b) \hat{K}_{d / 2+\tau+1}(z)-\frac{(d+2 \tau+1) \beta}{a^{2}} \hat{K}_{d / 2+\tau+1}(z)+\right. \\
& \left.+\frac{\beta \sin ^{2} \phi}{a^{2}} \hat{K}_{d / 2+\tau+2}(z)\right) \\
& J_{(0, \tau-1)}^{\left[z^{\prime}\right]} \simeq \frac{\Lambda_{d} \mathrm{e}^{-i(k b)}}{a^{2} \xi^{2}}\left[\left(\xi^{2}-\beta\right) \hat{K}_{d / 2+\tau}\left(z^{\prime}\right)-i \xi \cos \phi \hat{K}_{d / 2+\tau+1}\left(z^{\prime}\right)\right] .
\end{aligned}
$$

Representing $x=\left[x-\beta / \xi^{2}\right]+\beta / \xi^{2}$, we have

$$
J_{(1,-1)}=\frac{\beta}{\xi^{2}} J_{(0,-1)}+\frac{\Lambda_{d}}{\xi} \int_{0}^{1} r e^{-i(k b) x} \hat{K}_{d / 2-1}\left(\zeta_{n}\right) d x=\frac{\beta}{\xi^{2}} J_{(0,-1)}+\frac{\Lambda_{d}}{\xi} \bar{J},
$$

with notation

$$
\bar{J}=\int_{0}^{1} d x r(x) e^{-i(k b) x} \hat{K}_{d / 2-1}\left(\zeta_{n}\right), \quad r(x) \equiv \xi\left(x-\frac{\beta}{\xi^{2}}\right) .
$$

Representing [121, 122]

$$
\hat{K}_{\nu-\mu-1}\left(\sqrt{a^{2}-r^{2}}\right)=r^{-\mu} a^{\nu} \int_{0}^{\infty} \frac{y^{\mu+1} I_{\mu}(r y)}{\left(y^{2}+1\right)^{\nu / 2}} K_{\nu}\left(a \sqrt{y^{2}+1}\right) d y,
$$

we have

$$
\bar{J}=\int_{0}^{\infty} d y \frac{a^{\nu} y^{\mu+1}}{\left(y^{2}+1\right)^{\nu / 2}} K_{\nu}\left(a \sqrt{y^{2}+1}\right) \int_{0}^{1} d x e^{-i(k b) x} r^{1-\mu} I_{\mu}(r y) .
$$

Fix $\mu=1 / 2$, then $\nu=(d+1) / 2=(D-3) / 2>-1$, and

$$
r^{1 / 2} I_{1 / 2}(r y) y^{3 / 2}=y \frac{\sqrt{2}}{\sqrt{\pi}} \sinh (r y) .
$$


Taking the internal integral over $x$

$$
\int_{0}^{1} d x e^{-i(k b) x} \sinh \left(\xi\left[x-\beta / \xi^{2}\right] y\right)=\sum_{j=0,1}(-1)^{j-1} e^{-i(k b) j} \frac{y \xi \cosh \left(\xi d_{j} y\right)+i(k b) \sinh \left(\xi d_{j} y\right)}{\xi^{2} y^{2}+(k b)^{2}},
$$

with

$$
d_{j}=j-\beta / \xi^{2}=\delta_{1 j}-\beta / \xi^{2} \quad j=0,1
$$

we arrive at

$$
\begin{aligned}
\bar{J}=\frac{\sqrt{2}}{\sqrt{\pi}} a^{(d+1) / 2} & \sum_{j=0,1}(-1)^{j-1} e^{-i(k b) j} \times \\
& \times \int_{0}^{\infty} d y \frac{K_{(d+1) / 2}\left(a \sqrt{y^{2}+1}\right)}{\left(y^{2}+1\right)^{\nu / 2}} \frac{y^{2} \xi \cosh \xi d_{j} y+i y(k b) \sinh \xi d_{j} y}{\xi^{2} y^{2}+(k b)^{2}} .
\end{aligned}
$$

Substituting $(k b)=-\xi \cos \phi($ A.6) to get

$$
\bar{J}=\frac{\sqrt{2}}{\sqrt{\pi}} \frac{a^{\nu}}{\xi} \sum_{j=0,1}(-1)^{j-1} e^{-i(k b) j} \int_{0}^{\infty} d y \frac{K_{(d+1) / 2}\left(a \sqrt{y^{2}+1}\right)}{\left(y^{2}+1\right)^{\nu / 2}} \frac{y^{2} \cosh \xi d_{j} y-i y \cos \phi \sinh \xi d_{j} y}{y^{2}+\cos ^{2} \phi} .
$$

In the integrand numerator add and subtract $\cos ^{2} \phi$ to $y^{2}$ :

$$
\begin{aligned}
\bar{J}=\frac{\sqrt{2}}{\sqrt{\pi}} & \frac{a^{(d+1) / 2}}{\xi} \sum_{j=0,1}(-1)^{j-1} e^{-i(k b) j} \times \\
& \times \int_{0}^{\infty} d y \frac{K_{(d+1) / 2}\left(u \sqrt{y^{2}+1}\right)}{\left(y^{2}+1\right)^{(d+1) / 4}}\left[\cosh \xi d_{j} y-\frac{\cos ^{2} \phi \cosh \xi d_{j} y+i y \cos \phi \sinh \xi d_{j} y}{y^{2}+\cos ^{2} \phi}\right]
\end{aligned}
$$

and then, integrating $\cosh \xi d_{j} y$ in a bracket with help of (B.25) and comparing the remainder with

$$
\begin{aligned}
J_{(0,0)}=\frac{2^{1 / 2} a^{(d+1)}}{\pi^{1 / 2}} \frac{\Lambda_{d}}{\xi} \sum_{j=0,1} & (-1)^{j+1} e^{-i j(k b)} \times \\
& \times \int_{0}^{\infty} d y \hat{K}_{-(d+1) / 2}\left(a \sqrt{y^{2}+1}\right) \frac{y \sinh \left(\xi \delta_{j} y\right)-i \cos \phi \cosh \left(\xi \delta_{j} y\right)}{y^{2}+\cos ^{2} \phi}
\end{aligned}
$$

[106, eq. (3.28)], one concludes

$$
\bar{J}=\frac{1}{\xi}\left[e^{-i(k b)} \hat{K}_{d / 2}\left(z^{\prime}\right)-\hat{K}_{d / 2}(z)\right]-i \frac{\cos \phi}{\Lambda_{d}} J_{(0,0)} .
$$

Substituting into (B.23) one gets the recurrence relation:

$$
J_{(1,-1)}=\frac{\beta}{\xi^{2}} J_{(0,-1)}+\frac{\Lambda_{d}}{\xi^{2}}\left[e^{-i(k b)} \hat{K}_{d / 2}\left(z^{\prime}\right)-\hat{K}_{d / 2}(z)\right]-\frac{i \cos \phi}{\xi} J_{(0,0)} .
$$


The property is hold for all indices $(\tau \geqslant-1)$, thus we can generalize:

$$
\begin{aligned}
J_{(1, \tau)} & =\frac{\beta}{\xi^{2}} J_{(0, \tau)}+\frac{\Lambda_{d}}{\xi^{2}}\left[e^{i \xi \cos \phi} \hat{K}_{d / 2+\tau+1}\left(z^{\prime}\right)-\hat{K}_{d / 2+\tau+1}(z)\right]-\frac{i \cos \phi}{\xi} J_{(0, \tau+1)} \\
& =-\frac{i}{\xi} \frac{\partial J_{(0, \tau)}}{\partial(\cos \phi)}
\end{aligned}
$$

Splitting it onto $z-$ and $z^{\prime}-$ parts gives

$$
\begin{aligned}
& J_{(1, \tau)}^{[z]}=\frac{\beta}{\xi^{2}} J_{(0, \tau)}^{[z]}-\frac{\Lambda_{d}}{\xi^{2}} \hat{K}_{d / 2+\tau+1}(z)-\frac{i \cos \phi}{\xi} J_{(0, \tau+1)}^{[z]} \\
& J_{(1, \tau)}^{\left[z^{\prime}\right]}=\frac{\beta}{\xi^{2}} J_{(0, \tau)}^{\left[z^{\prime}\right]}+\frac{\Lambda_{d}}{\xi^{2}} e^{i \xi \cos \phi} \hat{K}_{d / 2+\tau+1}\left(z^{\prime}\right)-\frac{i \cos \phi}{\xi} J_{(0, \tau+1)}^{\left[z^{\prime}\right]} .
\end{aligned}
$$

Differentiating (B.31) over $\cos \phi$, one gets

$$
J_{(2, \tau)}=\frac{\beta}{\xi^{2}} J_{(1, \tau)}+\frac{\Lambda_{d}}{\xi^{2}} e^{i \xi \cos \phi} \hat{K}_{d / 2+\tau+1}\left(z^{\prime}\right)-\frac{1}{\xi^{2}} J_{(0, \tau+1)}-\frac{i \cos \phi}{\xi} J_{(1, \tau+1)} .
$$

Substituting (B.31)

$$
\begin{aligned}
J_{(2, \tau)}= & \frac{\beta^{2}}{\xi^{4}} J_{(0, \tau)}+\left.\frac{\Lambda_{d} \beta}{\xi^{2}} e^{i x \xi \cos \phi} \hat{K}_{d / 2+\tau+1}\left(\zeta_{n}\right)\right|_{0} ^{1}-\frac{2 i \beta \cos \phi}{\xi^{3}} J_{(0, \tau+1)}-\frac{1}{\xi^{2}} J_{(0, \tau+1)}+ \\
& +\frac{\Lambda_{d}}{\xi^{2}} e^{i \xi \cos \phi} \hat{K}_{d / 2+\tau+1}\left(z^{\prime}\right)-\left.\frac{i \Lambda_{d} \cos \phi}{\xi^{3}} e^{i x \xi \cos \phi} \hat{K}_{d / 2+\tau+2}\left(\zeta_{n}\right)\right|_{0} ^{1}-\frac{\cos ^{2} \phi}{\xi^{2}} J_{(0, \tau+2)}
\end{aligned}
$$

Splitting,

$$
\begin{aligned}
J_{(2, \tau)}^{[z]}= & \frac{\beta^{2}}{\xi^{4}} J_{(0, \tau)}^{[z]}-\frac{\Lambda_{d} \beta}{\xi^{2}} \hat{K}_{d / 2+\tau+1}(z)-\frac{2 i \beta \cos \phi}{\xi^{3}} J_{(0, \tau+1)}^{[z]}-\frac{1}{\xi^{2}} J_{(0, \tau+1)}^{[z]}+ \\
& +\frac{i \Lambda_{d} \cos \phi}{\xi^{3}} \hat{K}_{d / 2+\tau+2}(z)-\frac{\cos ^{2} \phi}{\xi^{2}} J_{(0, \tau+2)}^{[z]} \\
J_{(2, \tau)}^{\left[z^{\prime}\right]}= & \frac{\beta^{2}}{\xi^{4}} J_{(0, \tau)}^{\left[z^{\prime}\right]}+\Lambda_{d} \frac{1+\beta}{\xi^{2}} e^{i \xi \cos \phi} \hat{K}_{d / 2+\tau+1}\left(z^{\prime}\right)-\frac{2 i \beta \cos \phi}{\xi^{3}} J_{(0, \tau+1)}^{\left[z^{\prime}\right]}-\frac{1}{\xi^{2}} J_{(0, \tau+1)}^{\left[z^{\prime}\right]}- \\
& -\frac{i \Lambda_{d} \cos \phi}{\xi^{3}} e^{i \xi \cos \phi} \hat{K}_{d / 2+\tau+2}\left(z^{\prime}\right)-\frac{\cos ^{2} \phi}{\xi^{2}} J_{(0, \tau+2)}^{\left[z^{\prime}\right]} .
\end{aligned}
$$

Substituting (B.32) into (B.20) gives the full expansion of vectorial integral in terms of Macdonald functions.

\section{Integration over frequencies and angles}

(a) Here are computed the angular integrals of the general form

$$
V_{m}^{n}=\int_{0}^{\pi} \frac{\sin ^{n} \vartheta}{(1-v \cos \vartheta)^{m}} d \vartheta
$$


with integers $m, n$, which were encountered in the text. For $2 m>n+1$ one finds in the leading order [105]

$$
V_{m}^{n}=\frac{2^{m-1} \Gamma\left(\frac{n+1}{2}\right) \Gamma\left(m-\frac{n+1}{2}\right)}{\Gamma(m)} \gamma^{2 m-n-1} .
$$

For $2 m<n+1$ one obtains

$$
V_{m}^{n}=\frac{2^{n-m} \Gamma\left(\frac{n+1}{2}\right) \Gamma\left(\frac{n+1}{2}-m\right)}{\Gamma(n-m+1)} .
$$

In the case $2 m=n+1$ the behavior of the integral is logarithmic. The only one of those needed here is the:

$$
V_{2}^{3}=\frac{2}{v^{3}}\left[\ln \frac{1+v}{1-v}-2 v\right]=4(\ln 2 \gamma-1)+\mathcal{O}\left(\gamma^{-2}\right) .
$$

(b) Integration over the remaining angles is performed next. Since, with our choice of the coordinate system only one extra angle $\varphi$ enters the amplitudes, only the following formula is needed:

$$
\int_{S^{D-3}}|\sin \varphi|^{N} d \Omega=\frac{2 \pi^{(D-3) / 2}}{\Gamma\left(\frac{D-2+N}{2}\right)} \Gamma\left(\frac{N+1}{2}\right) .
$$

(c) Finally, computation of the integrals over the frequency or over the impact parameter involving two Macdonald functions of the same argument is performed using the formula [122]:

$$
\int_{0}^{\infty} K_{\mu}(c z) K_{\nu}(c z) z^{\alpha-1} d z=\frac{2^{\alpha-3} \Gamma\left(\frac{\alpha+\mu+\nu}{2}\right) \Gamma\left(\frac{\alpha+\mu-\nu}{2}\right) \Gamma\left(\frac{\alpha-\mu+\nu}{2}\right) \Gamma\left(\frac{\alpha-\mu-\nu}{2}\right)}{c^{\alpha} \Gamma(\alpha)} .
$$

\section{Asymptotic behavior of retarded fields}

In this appendix we derive the asymptotic behaviors of the retarded solutions of the gravitational wave equation in arbitrary dimensions and of their space-time derivatives.

The derivation is based on the well known formula [123-125] for the retarded Green's function of the $D$-dimensional d'Alembert operator

$$
G_{D}(x)= \begin{cases}\frac{1}{2 \pi^{D / 2-1}} \theta\left(x^{0}\right) \delta^{(D / 2-2)}\left(x^{2}\right), & D=\text { even } \\ \frac{1}{2 \pi^{(D-1) / 2}} \theta\left(x^{0}\right)\left(\frac{d}{d x^{2}}\right)^{\frac{D-3}{2}}\left(\frac{\theta\left(x^{2}\right)}{\left(x^{2}\right)^{1 / 2}}\right), & D=\text { odd }\end{cases}
$$

with $\delta^{(-1)}\left(x^{2}\right) \equiv \theta\left(x^{2}\right)$ and $x^{2}=\left(x^{0}\right)^{2}-\left(x^{1}\right)^{2}-\ldots-\left(x^{D-1}\right)^{2}$.

First, suppose we have one point particle, moving along the trajectory $\Gamma$, parametrized by $z^{M}(\tau)$ and properly normalized $\left(\dot{z}^{2}=1\right)$. The corresponding source of gravitational the field on flat background is $T^{M N}(x)=m \int \dot{z}^{M}(\tau) \dot{z}^{N}(\tau) \delta(x-z(\tau)) d \tau$. The solution of 
the d'Alembert equation $\square \psi_{\text {ret }}^{M N}=-\varkappa_{D} T^{M N}$ is the convolution $\psi^{M N}(x)=G_{\text {ret }} * T^{M N}$. Let us denote by $\hat{\tau}$ the retardation point on the smooth particle-worldline $\Gamma$ corresponding to a given observation point $x^{M}=(t, \mathbf{x})$. Given $x^{M}$ and the time-like trajectory $z^{M}(\tau)$, $\hat{\tau}$ is the unique solution (with $x^{0}>z^{0}(\hat{\tau})$ ) of the equation $(x-z(\tau))^{2}=0$, and specifies the intersection of $\Gamma$ with the past light-cone of the observation point $x^{M}$. Using hats to denote all corresponding kinematical quantities (e.g. $\hat{\dot{z}} \equiv \dot{z}(\hat{\tau})$ ), the Liénard-Wiechert solution becomes ${ }^{27}$

$$
\psi_{\text {ret }}^{M N}(x)=-\frac{\varkappa_{D} m}{4 \pi^{D / 2-1}}\left(\frac{1}{2 \rho} \frac{\partial}{\partial \hat{\tau}}\right)^{\frac{D-4}{2}} \frac{\hat{\dot{z}}^{M} \hat{\dot{z}}^{N}}{\rho}
$$

where

$$
\rho(x) \equiv-\frac{1}{2} \frac{\partial}{\partial \hat{\tau}}(x-\hat{z})^{2}=\hat{\dot{z}} \cdot(x-\hat{z})>0 .
$$

Note that in the co-moving at the retardation moment $\hat{\tau}$ Lorentz frame, the quantity $\rho(x)$ coincides with the spatial distance $|\mathbf{x}-\hat{\mathbf{z}}|$ between the observation and the retardation points.

Now, introduce the null vector $c^{M}=\left(x^{M}-\hat{z}^{M}\right) / \rho$. It represents the properly normalized vector from the retardation point towards the observation point. Introduce in addition the vector $n \equiv c-\hat{\dot{z}}$, which satisfies $n^{2}=-1$ and $n \cdot \hat{\dot{z}}=0$, as a consequence of $\hat{\dot{z}} \cdot c=1$ and $\hat{\dot{z}}^{2}=1$. Thus, $c^{M}$ is the sum of the orthogonal unit vectors: $\hat{\dot{z}}^{M}$, which specifies a time direction, and $n^{M}$ which is space-like and determines a spatial direction, i.e. a point on the sphere $S^{D-2}$, which is the "basis" of a null-cone with axis along $\hat{\dot{z}}^{M}$ and apex at $\hat{z}^{M}$. Thus, for an arbitrary point $x^{M}$ on this light cone, $\rho$ is the distance of the apex to the center of the sphere which contains $x^{M}$, and is also equal to the radius of that sphere. Thus, going to the wave-zone of radiation emitted by the accelerated particle corresponds to considering the limit $\rho \rightarrow+\infty$ keeping fixed the quantities $n, \hat{\dot{z}}$, $\hat{\ddot{z}}$, etc, which are related only to direction and refer to the apex $\hat{z}$ of the light-cone.

Now with the help of the relations $[126,127]$

$$
\begin{array}{rlrl}
\partial_{M} \hat{\tau} & =c_{M} & \dot{\rho} & =(\hat{\ddot{z}}) \rho-1 \\
\partial_{M} \rho & =\hat{\dot{z}}_{M}+\dot{\rho} c_{M} \quad \partial_{M} c^{N}=\frac{1}{\rho}\left(\delta_{M}^{N}-\hat{\dot{z}}_{M} c^{N}-c_{M} \hat{\dot{z}}^{N}-\dot{\rho} c_{M} c^{N}\right) \\
\partial_{M} \hat{\dot{z}}_{N} & =c_{M} \hat{\ddot{z}}_{N}, \quad \text { and similar ones for higher derivatives, }
\end{array}
$$

and consecutive-differentiation rule (D.2), one can check explicitly that in even dimensions the asymptotic behavior of $\psi^{M N}$ and its derivatives is

- $\psi_{D}^{M N}=\frac{\psi_{\text {Rad }}^{M N}}{\rho^{D / 2-1}}+\ldots+\frac{\psi_{\text {Newt }}^{M N}}{\rho^{D-3}}$, with $\psi_{\text {Rad }}^{M N}, \ldots, \psi_{\text {Newt }}^{M N}-$ tensors depending on
$c, \hat{\dot{z}}, \hat{\ddot{z}}, \ldots$

${ }^{27}$ The square root derivative, which appears in odd-dimensions, is defined as the convolution

$$
(d / d x)^{1 / 2} f(x)=-\frac{1}{2 \sqrt{\pi}} \int_{0}^{x} \frac{d t}{t^{3 / 2}} f(x-t) .
$$


- $\psi_{D}^{M N, P}=\frac{\Psi_{\mathrm{Rad}}^{M N P}}{\rho^{D / 2-1}}+\ldots+\frac{\Psi_{\mathrm{N} \text { Net }}^{M N P}}{\rho^{D-2}}$ with $\Psi_{\mathrm{Rad}}^{M N P}, \ldots, \Psi_{\text {Newt }}^{M N P}$ - tensors depending on $c, \hat{\dot{z}}, \hat{\ddot{z}}, \ldots$ The same asymptotic behavior holds for higher order space-time derivatives, but not for $\square \psi^{M N}$, for which one obtains instead

- $\square \psi_{D}^{M N}=\frac{\tilde{\tilde{\psi}}_{\text {Rad }}^{M N}}{\rho^{D / 2}}+\ldots+\frac{\tilde{\tilde{\psi}}_{\text {Newt }}^{M N}}{\rho^{D-1}}$.

In odd dimensions, on the other hand, the solutions of d'Alembert equations are not known in closed form and one has to rely on approximate asymptotic expansions. In particular, one can use the expansion (proven for scalar and vector fields in [128-130] and conjectured for the tensor field $\psi^{M N}$ )

$$
\psi_{D}^{M N}=\frac{\psi_{\mathrm{Rad}}^{M N}}{\rho^{D / 2-1}}+\mathcal{O}\left(\rho^{-D / 2}\right)
$$

where $\psi_{\text {Rad }}^{M N}$ again depends on $c^{M}$ and on the retarded quantities $\hat{\dot{z}}, \hat{\ddot{z}}$ etc, but is expressed as an integral along the particle trajectory from $\tau^{\prime}=-\infty$ to $\tau^{\prime}=\hat{\tau}$. Taking into account that $c^{M}$ enters into $\psi_{\operatorname{Rad}}^{M N}$ either in the product $\left(\hat{z}-z\left(\tau^{\prime}\right)\right) \cdot c$ or with a free index, one can apply (D.3) to the integral and, using $c^{2}=0$ to obtain the same behavior as in even dimensions, namely: ${ }^{28}$

$$
\square \frac{\psi_{\mathrm{Rad}}^{M N}}{\rho^{D / 2-1}}=\mathcal{O}\left(\rho^{-D / 2}\right) \quad \text { and } \quad \square \psi_{D}^{M N}=\mathcal{O}\left(\rho^{-D / 2}\right) .
$$

For full gravity these properties do not hold exactly due to non-linearities, but to the order discussed here we have effective linear theory with linear operator $\square$ and sources ${ }^{1} T,{ }^{1} T^{\prime}$ and $S\left({ }^{1} h,{ }^{1} h^{\prime}\right)$, respectively. The first two are local currents and satisfy the assumptions made above. $S\left({ }^{1} h\right)$, on the other hand, is not a current generated by the point-like source, but, expressed explicitly with the help of (A.5), and taking into account the field equation (2.23), one can easily check that this part of $\square^{2} \psi_{M N}$ falls-off much faster at large distances, namely as the product of two Coulomb fields,

$$
\square^{2} \psi_{M N}(S)=-\varkappa_{D} S_{M N} \sim{ }^{1} h^{1} h^{\prime}=\mathcal{O}\left(\rho^{-(2 D-6)}\right) .
$$

Thus, when substituted to replace $\square^{2} \psi_{M N}$ into the integrals

$$
\int_{C}{ }^{2} h^{P Q} \square^{2} \psi_{M P} d \sigma_{Q} \quad \text { and } \quad \int_{C}{ }^{2} h \square^{2} \psi_{M P} d \sigma^{P}
$$

${ }^{28}$ Relations (D.1) can be combined into the single formula

$$
G_{D}^{\mathrm{ret}}\left(x_{D}^{2}\right)=\left.\theta\left(x^{0}\right)\left(\frac{1}{\sqrt{\pi}} \frac{\partial}{\partial y}\right)^{\frac{D-2}{2}} G_{2}(y)\right|_{y=x_{D}^{2}},
$$

where $G_{2}(y)=\theta(y) / 2$ and $x_{D}^{2}=x_{0}^{2}-x_{1}^{2}-\ldots-x_{D-1}^{2}$. Already from (D.1) one may notice that the solution $\psi_{D+2}$ of d'Alembert equation in $D+2$ dimensions is proportional to $\square \psi_{D}$. Thus if $\psi_{D}^{M N}$ scales in the wave-zone as $1 / \rho^{D / 2-1}, \square \psi_{D}^{M N}$ should scale as $1 / \rho^{(D+2) / 2-1}=1 / \rho^{D / 2}$, in agreement with (D.5). 
encountered in section 2, the resulting integrals vanish as a result of the asymptotic behaviors (D.5) and (D.6). In other words, $S_{M N}$ is significant only in the space-time volume in which both ${ }^{1} h$ and ${ }^{1} h^{\prime}$ are important, i.e. close to the moment of collision. This justifies the use of the asymptotic expansions presented above.

Open Access. This article is distributed under the terms of the Creative Commons Attribution License which permits any use, distribution and reproduction in any medium, provided the original author(s) and source are credited.

\section{References}

[1] N. Arkani-Hamed, S. Dimopoulos and G. Dvali, The Hierarchy problem and new dimensions at a millimeter, Phys. Lett. B 429 (1998) 263 [hep-ph/9803315] [INSPIRE].

[2] I. Antoniadis, N. Arkani-Hamed, S. Dimopoulos and G. Dvali, New dimensions at a millimeter to a Fermi and superstrings at a TeV, Phys. Lett. B 436 (1998) 257 [hep-ph/9804398] [INSPIRE].

[3] N. Arkani-Hamed, S. Dimopoulos and G. Dvali, Phenomenology, astrophysics and cosmology of theories with submillimeter dimensions and $\mathrm{TeV}$ scale quantum gravity, Phys. Rev. D 59 (1999) 086004 [hep-ph/9807344] [INSPIRE].

[4] G.F. Giudice, R. Rattazzi and J.D. Wells, Quantum gravity and extra dimensions at high-energy colliders, Nucl. Phys. B 544 (1999) 3 [hep-ph/9811291] [INSPIRE].

[5] T. Han, J.D. Lykken and R.-J. Zhang, On Kaluza-Klein states from large extra dimensions, Phys. Rev. D 59 (1999) 105006 [hep-ph/9811350] [INSPIRE].

[6] J. Tanaka, T. Yamamura, S. Asai and J. Kanzaki, Study of black holes with the ATLAS detector at the LHC, Eur. Phys. J. C 41 (2005) 19 [hep-ph/0411095] [INSPIRE].

[7] C. Harris, M. Palmer, M.A. Parker, P. Richardson, A. Sabetfakhri, et al., Exploring higher dimensional black holes at the large hadron collider, JHEP 05 (2005) 053 [hep-ph/0411022] [INSPIRE].

[8] L. Lönnblad, M. Sjodahl and T. Akesson, QCD-suppression by black hole production at the LHC, JHEP 09 (2005) 019 [hep-ph/0505181] [INSPIRE].

[9] B. Koch, M. Bleicher and S. Hossenfelder, Black hole remnants at the LHC, JHEP 10 (2005) 053 [hep-ph/0507138] [INSPIRE].

[10] L. Lönnblad and M. Sjodahl, Classical and non-classical ADD-phenomenology with high-E (perpendicular) jet observables at collider experiments, JHEP 10 (2006) 088 [hep-ph/0608210] [INSPIRE].

[11] B. Koch, M. Bleicher and H. Stoecker, Black Holes at LHC?, J. Phys. G 34 (2007) S535 [hep-ph/0702187] [INSPIRE].

[12] G. 't Hooft, Graviton Dominance in Ultrahigh-Energy Scattering, Phys. Lett. B 198 (1987) 61 [INSPIRE].

[13] G.F. Giudice, R. Rattazzi and J.D. Wells, Transplanckian collisions at the LHC and beyond, Nucl. Phys. B 630 (2002) 293 [hep-ph/0112161] [INSPIRE].

[14] R. Emparan, M. Masip and R. Rattazzi, Cosmic rays as probes of large extra dimensions and TeV gravity, Phys. Rev. D 65 (2002) 064023 [hep-ph/0109287] [INSPIRE]. 
[15] P.C. Argyres, S. Dimopoulos and J. March-Russell, Black holes and submillimeter dimensions, Phys. Lett. B 441 (1998) 96 [hep-th/9808138] [INSPIRE].

[16] T. Banks and W. Fischler, A Model for high-energy scattering in quantum gravity, hep-th/9906038 [INSPIRE].

[17] S.B. Giddings and S.D. Thomas, High-energy colliders as black hole factories: The End of short distance physics, Phys. Rev. D 65 (2002) 056010 [hep-ph/0106219] [INSPIRE].

[18] S. Dimopoulos and G.L. Landsberg, Black holes at the LHC, Phys. Rev. Lett. 87 (2001) 161602 [hep-ph/0106295] [INSPIRE].

[19] S.N. Solodukhin, Classical and quantum cross-section for black hole production in particle collisions, Phys. Lett. B 533 (2002) 153 [hep-ph/0201248] [INSPIRE].

[20] A. Jevicki and J. Thaler, Dynamics of black hole formation in an exactly solvable model, Phys. Rev. D 66 (2002) 024041 [hep-th/0203172] [INSPIRE].

[21] S.C. Park and H. Song, Production of spinning black holes at colliders, J. Korean Phys. Soc. 43 (2003) 30 [hep-ph/0111069] [INSPIRE].

[22] A.V. Kotwal and C.P. Hays, Production and decay of spinning black holes at colliders and tests of black hole dynamics, Phys. Rev. D 66 (2002) 116005 [hep-ph/0206055] [INSPIRE].

[23] L.A. Anchordoqui, J.L. Feng, H. Goldberg and A.D. Shapere, Black holes from cosmic rays: Probes of extra dimensions and new limits on TeV scale gravity, Phys. Rev. D 65 (2002) 124027 [hep-ph/0112247] [INSPIRE].

[24] M. Cavaglia, Black hole and brane production in TeV gravity: A Review, Int. J. Mod. Phys. A 18 (2003) 1843 [hep-ph/0210296] [inSPIRE].

[25] P. Kanti, Black holes in theories with large extra dimensions: A Review, Int. J. Mod. Phys. A 19 (2004) 4899 [hep-ph/0402168] [INSPIRE].

[26] D.M. Gingrich, Black hole cross-section at the large hadron collider, Int. J. Mod. Phys. A 21 (2006) 6653 [hep-ph/0609055] [INSPIRE].

[27] B. Koch, M. Bleicher and H. Stoecker, Black Holes at LHC?, J. Phys. G 34 (2007) S535 [hep-ph/0702187] [INSPIRE].

[28] M. Cavaglià, Black hole and brane production in TeV gravity: A Review, Int. J. Mod. Phys. A 18 (2003) 1843 [hep-ph/0210296] [inSPIRE].

[29] G.L. Landsberg, Black Holes at Future Colliders and Beyond, J. Phys. G 32 (2006) R337 [hep-ph/0607297] [INSPIRE].

[30] R. Emparan, Black hole production at a TeV, hep-ph/0302226 [INSPIRE].

[31] P. Kanti, Black holes in theories with large extra dimensions: A Review, Int. J. Mod. Phys. A 19 (2004) 4899 [hep-ph/0402168] [InSPIRE].

[32] V.S. Rychkov, Topics in black hole production, Cargèse Summer School, June 7-19, 2004, 363 [hep-th/0410295] [INSPIRE].

[33] S. Hossenfelder, What black holes can teach us, hep-ph/0412265 [INSPIRE].

[34] T.G. Rizzo, TeV-scale black holes in warped higher-curvature gravity, hep-ph/0510420 [INSPIRE].

[35] D.M. Eardley and S.B. Giddings, Classical black hole production in high-energy collisions, Phys. Rev. D 66 (2002) 044011 [gr-qc/0201034] [INSPIRE]. 
[36] S.B. Giddings and V.S. Rychkov, Black holes from colliding wavepackets, Phys. Rev. D 70 (2004) 104026 [hep-th/0409131] [INSPIRE].

[37] F.S. Coelho, C. Herdeiro, C. Rebelo and M. Sampaio, Radiation from a D-dimensional collision of shock waves: higher order set up and perturbation theory validity, arXiv:1206.5839 [INSPIRE].

[38] P.D. D'Eath, High Speed Black Hole Encounters and Gravitational Radiation, Phys. Rev. D 18 (1978) 990 [INSPIRE].

[39] P.D. D'Eath and P. Payne, Gravitational radiation in high speed black hole collisions. 1. Perturbation treatment of the axisymmetric speed of light collision, Phys. Rev. D 46 (1992) 658 [InSPIRE].

[40] P.D. D'Eath and P. Payne, Gravitational radiation in high speed black hole collisions. 2. Reduction to two independent variables and calculation of the second order news function, Phys. Rev. D 46 (1992) 675 [inSPIRE].

[41] P.D. D'Eath and P. Payne, Gravitational radiation in high speed black hole collisions. 3. Results and conclusions, Phys. Rev. D 46 (1992) 694 [InSPIRE].

[42] P.D. D'Eath, Black holes: Gravitational interactions, in Oxford mathematical monographs, Clarendon, Oxford, U.K. (1996) 286 p.

[43] K. Glampedakis and D. Kennefick, Zoom and whirl: Eccentric equatorial orbits around spinning black holes and their evolution under gravitational radiation reaction, Phys. Rev. D 66 (2002) 044002 [gr-qc/0203086] [INSPIRE].

[44] F. Pretorius and D. Khurana, Black hole mergers and unstable circular orbits, Class. Quant. Grav. 24 (2007) S83 [gr-qc/0702084] [inSPIRE].

[45] L. Smarr, A. Cadez, B.S. DeWitt and K. Eppley, Collision of Two Black Holes: Theoretical Framework, Phys. Rev. D 14 (1976) 2443 [InSPIRE].

[46] L. Smarr, Gravitational Radiation from Distant Encounters and from Headon Collisions of Black Holes: The Zero Frequency Limit, Phys. Rev. D 15 (1977) 2069 [inSPIRE].

[47] R.A. Matzner and Y. Nutku, On the method of virtual quanta and gravitational radiation, Proc. Roy. Soc. Lond. 336 (1974) 285 [InSPIRE].

[48] R.A. Matzner, The Method of Virtual Quanta as a Probe of the Equivalence Principle, Gen. Rel. Grav. 9 (1978) 71 [inSPIRE].

[49] V. Cardoso, O.J. Dias and J.P. Lemos, Gravitational radiation in D-dimensional space-times, Phys. Rev. D 67 (2003) 064026 [hep-th/0212168] [INSPIRE].

[50] V. Cardoso, J.P. Lemos and S. Yoshida, Electromagnetic radiation from collisions at almost the speed of light: An Extremely relativistic charged particle falling into a Schwarzschild black hole, Phys. Rev. D 68 (2003) 084011 [gr-qc/0307104] [INSPIRE].

[51] E. Berti, M. Cavaglia and L. Gualtieri, Gravitational energy loss in high-energy particle collisions: Ultrarelativistic plunge into a multidimensional black hole, Phys. Rev. D 69 (2004) 124011 [hep-th/0309203] [INSPIRE].

[52] V. Cardoso, M. Cavaglia and J.-Q. Guo, Gravitational Larmor formula in higher dimensions, Phys. Rev. D 75 (2007) 084020 [hep-th/0702138] [INSPIRE].

[53] V. Cardoso, E. Berti and M. Cavaglia, What we (don't) know about black hole formation in high-energy collisions, Class. Quant. Grav. 22 (2005) L61 [hep-ph/0505125] [INSPIRE]. 
[54] B.M. Barker, S. Gupta and J. Kaskas, Graviton bremsstrahlung and infrared divergence, Phys. Rev. 182 (1969) 1391 [INSPIRE].

[55] B.M. Barker and S. Gupta, High-energy graviton bremsstrahlung, Phys. Rev. D 9 (1974) 334 [InSPIRE].

[56] B.M. Barker and R. O'Connell, Gravitational Two-Body Problem with Arbitrary Masses, Spins and Quadrupole Moments, Phys. Rev. D 12 (1975) 329 [INSPIRE].

[57] D.V. Galtsov, Y. Grats and A. Matyukhin, Problem of bremsstrahlung in the case of gravitational interaction, Sov. Phys. J. 23 (1980) 389 [INSPIRE].

[58] W.D. Goldberger and I.Z. Rothstein, An Effective field theory of gravity for extended objects, Phys. Rev. D 73 (2006) 104029 [hep-th/0409156] [INSPIRE].

[59] R.A. Porto and I.Z. Rothstein, The Hyperfine Einstein-Infeld-Hoffmann potential, Phys. Rev. Lett. 97 (2006) 021101 [gr-qc/0604099] [INSPIRE].

[60] R.A. Porto and R. Sturani, Scalar gravity: Post-Newtonian corrections via an effective field theory approach, gr-qc/0701105 [INSPIRE].

[61] W.D. Goldberger and I.Z. Rothstein, Dissipative effects in the worldline approach to black hole dynamics, Phys. Rev. D 73 (2006) 104030 [hep-th/0511133] [INSPIRE].

[62] W.D. Goldberger, Les Houches lectures on effective field theories and gravitational radiation, hep-ph/0701129 [INSPIRE].

[63] B. Kol and M. Smolkin, Classical Effective Field Theory and Caged Black Holes, Phys. Rev. D 77 (2008) 064033 [arXiv:0712.2822] [INSPIRE].

[64] B. Kol and M. Smolkin, Non-Relativistic Gravitation: From Newton to Einstein and Back, Class. Quant. Grav. 25 (2008) 145011 [arXiv:0712.4116] [INSPIRE].

[65] J.B. Gilmore and A. Ross, Effective field theory calculation of second post-Newtonian binary dynamics, Phys. Rev. D 78 (2008) 124021 [arXiv:0810.1328] [INSPIRE].

[66] C.R. Galley and M. Tiglio, Radiation reaction and gravitational waves in the effective field theory approach, Phys. Rev. D 79 (2009) 124027 [arXiv:0903.1122] [INSPIRE].

[67] D. Amati, M. Ciafaloni and G. Veneziano, Effective action and all order gravitational eikonal at Planckian energies, Nucl. Phys. B 403 (1993) 707 [InSPIRE].

[68] E. Kohlprath and G. Veneziano, Black holes from high-energy beam-beam collisions, JHEP 06 (2002) 057 [gr-qc/0203093] [INSPIRE].

[69] D. Amati, M. Ciafaloni and G. Veneziano, Towards an S-matrix description of gravitational collapse, JHEP 02 (2008) 049 [arXiv: 0712.1209] [INSPIRE].

[70] G. Veneziano and J. Wosiek, Exploring an S-matrix for gravitational collapse, JHEP 09 (2008) 023 [arXiv:0804.3321] [INSPIRE].

[71] G. Veneziano and J. Wosiek, Exploring an S-matrix for gravitational collapse. II. A Momentum space analysis, JHEP 09 (2008) 024 [arXiv: 0805.2973] [INSPIRE].

[72] M. Ciafaloni and D. Colferai, S-matrix and Quantum Tunneling in Gravitational Collapse, JHEP 11 (2008) 047 [arXiv:0807.2117] [INSPIRE].

[73] B. Koch and M. Bleicher, Gravitational radiation from elastic particle scattering in models with extra dimensions, JETP Lett. 87 (2008) 67 [hep-th/0512353] [INSPIRE]. 
[74] B. Koch, H.-J. Drescher and M. Bleicher, Gravitational radiation from ultrahigh energy cosmic rays in models with large extra dimensions, Astropart. Phys. 25 (2006) 291 [astro-ph/0602164] [INSPIRE].

[75] P. Anninos, D. Hobill, E. Seidel, L. Smarr and W.-M. Suen, The Collision of two black holes, Phys. Rev. Lett. 71 (1993) 2851 [gr-qc/9309016] [InSPIRE].

[76] P. Anninos, R.H. Price, J. Pullin, E. Seidel and W.-M. Suen, Headon collision of two black holes: Comparison of different approaches, Phys. Rev. D 52 (1995) 4462 [gr-qc/9505042] [INSPIRE].

[77] P. Anninos, D. Hobill, E. Seidel, L. Smarr and W.-M. Suen, The Headon collision of two equal mass black holes, Phys. Rev. D 52 (1995) 2044 [gr-qc/9408041] [InSPIRE].

[78] J.G. Baker, A. Abrahams, P. Anninos, S. Brandt, R. Price, et al., The Collision of boosted black holes, Phys. Rev. D 55 (1997) 829 [gr-qc/9608064] [InSPIRE].

[79] Binary Black Hole Grand Challenge Alliance collaboration, A. Abrahams et al., Gravitational wave extraction and outer boundary conditions by perturbative matching, Phys. Rev. Lett. 80 (1998) 1812 [gr-qc/9709082] [INSPIRE].

[80] M.W. Choptuik and F. Pretorius, Ultra Relativistic Particle Collisions, Phys. Rev. Lett. 104 (2010) 111101 [arXiv:0908.1780] [inSPIRE].

[81] U. Sperhake, V. Cardoso, F. Pretorius, E. Berti and J.A. Gonzalez, The High-energy collision of two black holes, Phys. Rev. Lett. 101 (2008) 161101 [arXiv:0806.1738] [INSPIRE].

[82] H. Yoshino and Y. Nambu, Black hole formation in the grazing collision of high-energy particles, Phys. Rev. D 67 (2003) 024009 [gr-qc/0209003] [inSPIRE].

[83] H. Yoshino and V.S. Rychkov, Improved analysis of black hole formation in high-energy particle collisions, Phys. Rev. D 71 (2005) 104028 [Erratum ibid. D 77 (2008) 089905] [hep-th/0503171] [INSPIRE].

[84] H. Yoshino and R.B. Mann, Black hole formation in the head-on collision of ultrarelativistic charges, Phys. Rev. D 74 (2006) 044003 [gr-qc/0605131] [INSPIRE].

[85] H. Yoshino, T. Shiromizu and M. Shibata, The Close limit analysis for head-on collision of two black holes in higher dimensions: Brill-Lindquist initial data,

Phys. Rev. D 72 (2005) 084020 [gr-qc/0508063] [INSPIRE].

[86] H. Yoshino, T. Shiromizu and M. Shibata, Close-slow analysis for head-on collision of two black holes in higher dimensions: Bowen-York initial data, Phys. Rev. D 74 (2006) 124022 [gr-qc/0610110] [INSPIRE].

[87] G. Marchesini and E. Onofri, High energy gravitational scattering: A Numerical study, JHEP 06 (2008) 104 [arXiv:0803.0250] [INSPIRE].

[88] M. Siino, Head-on collision and merging entropy of black holes: Reconsideration of Hawking's inequality, arXiv:0909.4827 [INSPIRE].

[89] K.S. Thorne and S.J. Kovacs, The generation of gravitational waves. I. Weak-field sources, Astrophys. J. 200 (1975) 245.

[90] R. Crowley and K. Thorne, The Generation of Gravitational Waves. 2. The Postlinear Formalism Revisited, Astrophys. J. 215 (1977) 624 [inSPIRE]. 
[91] S.J. Kovacs and K. Thorne, The Generation of Gravitational Waves. 3. Derivation of Bremsstrahlung Formulas, Astrophys. J. 217 (1977) 252 [INSPIRE].

[92] S.J. Kovacs and K. Thorne, The Generation of Gravitational Waves. 4. Bremsstrahlung, Astrophys. J. 224 (1978) 62 [INSPIRE].

[93] P. Havas, Radiation Damping in General Relativity, Phys. Rev. 108 (1957) 1351.

[94] B. Bertotti, Theory of gravitational perturbations in the fast motion approximations, J. Plebansky Ann. of Phys. 11 (1960) 169.

[95] J.N. Goldberg, in Gravitation; An Introduction to the Current Reseach, New York, U.S.A. (1962) 102.

[96] P. Havas and J.N. Goldberg, Lorentz-Invariant Equations of Motion of Point Masses in the General Theory of Relativity, Phys. Rev. B 128 (1962) 398.

[97] D. Robaschik, On perturbative calculations in gravitational theory, Acta Phys. Polon. 24 (1963) 299.

[98] S.F. Smith and P. Havas, Effects of Gravitational Radiation Reaction in the General Relativistic Two-Body Problem by a Lorentz-Invariant Approximation Method, Phys. Rev. B 138 (1965) 495.

[99] L. Infeld and R. Michalska-Trautman, The two-body problem and gravitational radiation, Annals Phys. 55 (1969) 561 [INSPIRE].

[100] H. Okamura, T. Ohta, T. Kimura and K. Hiida, Perturbation calculation of gravitational potentials, Prog. Theor. Phys. 50 (1973) 2066 [InSPIRE].

[101] P.C. Peters, Relativistic gravitational bremsstrahlung, Phys. Rev. D 1 (1970) 1559 [INSPIRE].

[102] P.C. Peters, Gravitational radiation from relativistic systems, Phys. Rev. D 5 (1972) 2476 [INSPIRE].

[103] P.C. Peters, Extreme relativistic limit for gravitational radiation from an electromagnetically bound system - errata and addenda, Phys. Rev. D 8 (1973) 4628 [INSPIRE].

[104] E. Berti et al., Semianalytical estimates of scattering thresholds and gravitational radiation in ultrarelativistic black hole encounters, Phys. Rev. D 81 (2010) 104048 [arXiv: 1003.0812] [INSPIRE].

[105] D.V. Galtsov, G. Kofinas, P. Spirin and T.N. Tomaras, Classical ultrarelativistic bremsstrahlung in extra dimensions, JHEP 05 (2010) 055 [arXiv: 1003.2982] [INSPIRE].

[106] Y. Constantinou, D. Gal'tsov, P. Spirin and T.N. Tomaras, Scalar Bremsstrahlung in Gravity-Mediated Ultrarelativistic Collisions, JHEP 11 (2011) 118 [arXiv:1106.3509] [INSPIRE].

[107] D.V. Gal'tsov, G. Kofinas, P. Spirin and T.N. Tomaras, Transplanckian bremsstrahlung and black hole production, Phys. Lett. B 683 (2010) 331 [arXiv:0908.0675] [INSPIRE].

[108] A. Mironov and A. Morozov, Is Strong Gravitational Radiation predicted by TeV-Gravity?, Pisma Zh. Eksp. Teor. Fiz. 85 (2007) 9 [hep-ph/0612074] [INSPIRE].

[109] A. Mironov and A. Morozov, Radiation beyond four space-time dimensions, Theor. Math. Phys. 156 (2008) 1209 [hep-th/0703097] [INSPIRE]. 
[110] A. Mironov and A. Morozov, On the problem of radiation friction beyond 4 and 6 dimensions, Int. J. Mod. Phys. A 23 (2008) 4677 [arXiv:0710.5676] [INSPIRE].

[111] D. Galakhov, Self-interaction and regularization of classical electrodynamics in higher dimensions, JETP Lett. 87 (2008) 452 [arXiv:0710.5688] [INSPIRE].

[112] S. Weinberg, Gravitation and Cosmology: Principles and Applications of the General Theory of Relativity, Wiley (1972).

[113] P.C. Aichelburg and R.U. Sexl, On the Gravitational field of a massless particle, Gen. Rel. Grav. 2 (1971) 303 [InSPIRE].

[114] I.B. Khriplovich and E.V. Shuryak, Radiation emitted by an ultrarelativistic particle in a gravitational field, Zh. Eksp. Teor. Fiz. 65 (1973) 2137 [INSPIRE].

[115] D.V. Galtsov and A.A. Matyukhin, Radiation from ultrarelativistic particles in strong gravitational fields (in russian), Yad. Fiz. 45 (1987) 894 [INSPIRE].

[116] D.V. Galtsov, Radiation reaction in various dimensions, Phys. Rev. D 66 (2002) 025016 [hep-th/0112110] [INSPIRE].

[117] D.V. Gal'tsov and P.A. Spirin, Radiation reaction in curved even-dimensional spacetime, Grav. Cosmol. 13 (2007) 241.

[118] D. Gal'tsov, P. Spirin and S. Staub, Radiation reaction in curved space-time: Local method, in Gravitation and Astrophysics, J.M. Nester, C.-M. Chen and J.-P. Hsu eds., World Scientific (2006) p. 345 [gr-qc/0701004] [INSPIRE].

[119] S. Weinberg, Infrared photons and gravitons, Phys. Rev. B 140 (1965) 516.

[120] D.V. Gal'tsov, G. Kofinas, P. Spirin and T.N. Tomaras, Classical ultra-relativistic scattering in $A D D$, JHEP 05 (2009) 074 [arXiv:0903.3019] [INSPIRE].

[121] I.S. Gradshteyn and I.M. Ryzhik, Table of Integrals, Series and Products, Academic Press, (1965).

[122] A.P. Prudnikov, Yu.A. Brychkov and O.I. Marichev, Integrals and Series, Vol. 1, Elementary Functions, Gordon \& Breach Sci. Publ., New York, U.S.A. (1986).

[123] V.S. Vladimirov, Equations of Mathematical Physics, Dekker, New York (1971).

[124] V. Vladimirov and I. Petrova, Distributions en physique mathématique, (in French), Moscow, URSS (2008).

[125] I.M. Gel'fand and G.E. Shilov, Generalized Functions, Vol.I, Academic Press (1964).

[126] B.P. Kosyakov, Exact solutions of classical electrodynamics and the Yang-Mills-Wong theory in even-dimensional space-time, Theor. Math. Phys. 199 (1999) 493.

[127] D.V. Gal'tsov and P. Spirin, Radiation reaction reexamined: Bound momentum and Schott term, Grav. Cosmol. 12 (2006) 1 [hep-th/0405121] [INSPIRE].

[128] P. Spirin, Massless field emission in the space-time of extra dimensions, Grav. Cosmol. 15 (2009) 82.

[129] P.Spirin, Radiation reaction and renormalization in curved space-times of various dimensions, Ph.D. thesis, Moscow State University (2008).

[130] D.V. Gal'tsov and P. Spirin, to appear. 